\title{
Uso de métodos Bayesianos para modelos com pontos de mudança
}

\author{
Selene Maria Coelho Loibel
}

\section{Orientador: Prof. Dr. Jorge Alberto Achcar \\ ICMC-USP}

\footnotetext{
Dissertação apresentada ao Instituto de Ciências Matemáticas e de Computação - USP, como parte dos requisitos para obtenção do titulo de Mestre em Ciências - Área de Ciências de Computação e Matemática Computacional
} 
Aos meus pais,

Izette e Gilberto

e ao meu filho Diogo. 
Agradecimentos

A conclusão dessa dissertação não seria possivel sem o auxílio de algumas pessoas e instituições, aos quais expresso meu sincero agradecimento.

Ao professor Dr. Jorge Alberto Achcar pela orientação segura e dedicada em todo decorrer da pesquisa.

Ao profesor Dr. Josemar Rodrigues e ao professor Dr. Carlos Diniz pelas sugestões dadas no meu exame da qualificação.

Ao professor Dr. Marinho Gomes de Andrade pela imprescindível colaboração à este trabalho.

À FAPESP pelo apoio financeiro.

Aos professores, funcionários e colegas de pós-graduação que de alguma maneira contribuiram para a realização deste projeto. 


\section{Índice}

Capitulo 1 - Introdução

1.1 Apresentação do problema 1

1.2 Revisão bibliográfica 2

1.3 Conteúdo da dissertação

Capítulo 2 - Modelos de sobrevivência com função de risco constante 6

2.1 Apresentação dos modelos 6

2.1.1 Modelo exponencial com um ponto de mudança - um tratamento 7

2.1.2 Modelo exponencial com um ponto de mudança - dois tratamentos 7

2.2 Inferência clássica $\quad 8$

2.2.1 Análise para o modelo exponencial com um tratamento 9

2.2.2 Análise para o modelo exponencial com dois tratamentos $\quad 12$

2.3 Inferência Bayesiana 15

2.3.1 Análise para o modelo exponencial com um tratamento 16

2.3.2 Análise para o modelo exponencial com dois tratamentos 23

Capitulo 3 - Modelo de sobrevivência com função de risco variável 30

3.1 Apresentação do modelo 30

3.1.1 Modelo Weibull com um ponto de mudança - Um tratamento 30

3.2 Inferência clássica 31

3.3 Inferência Bayesiana 34

Capítulo 4 - Processo de Poisson com pontos de mudança 37

4.1 Apresentação dos modelos 37

4.1.1 Processo de Poisson com um ponto de mudança 37

4.1.2 Processo de Poisson com dois pontos de mudança 38

4.2 Inferência clássica $\quad 38$ 
4.3 Inferência Bayesiana $\quad 39$

4.3.1 Análise do processo com um ponto de mudança

4.3.2 Análise do processo com dois pontos de mudança 40

4.3.3 Análise de um modelo hierárquico para o processo com um ponto de mudança

Capítulo 5 - Aplicação dos modelos propostos

5.1 Aplicação para o modelo exponencial com um tratamento

5.2 Aplicação para o modelo exponencial com dois tratamentos

5.3 Aplicação para o modelo Weibull com um tratamento

5.4 Comparação dos modelos de sobrevivência

5.5 Aplicação para o processo de Poisson pontos de mudança

5.5.1 Modelo com um ponto de mudança conhecido

5.5.2 Modelo com um ponto de mudança desconhecido $\left(M_{1}\right)$

5.5.3 Modelo com dois pontos de mudança desconhecidos $\left(M_{2}\right)$

5.5.4 Comparação dos modelos $M_{1}$ e $M_{2}$

Conclusőes e propostas futuras

Apêndice

Referências 


\section{Resumo}

Neste trabalho apresentamos uma análise Bayesiana para modelos de sobrevivência e de contagem com pontos de mudança, assumindo diferentes densidades a priori e métodos computacionais para obter as inferências a posteriori de interesse. Em particular, destacamos o método de Laplace e métodos de simulação de Monte Carlo em cadeias de Markov. Além disso introduzimos um critério de seleção de modelos Bayesiano. Ilustramos a metodologia proposta em alguns exemplos numéricos. 


\section{Abstract}

In this work we present a Bayesian analysis for survival and counting models with change-points, assuming different prior densities and computational methods to obtain the posterior summaries of interest. In special, we consider Laplace's method and Monte Carlo Markov chain methods. We also introduce some Bayesian criteria to discriminate the proposed models. We illustrate the proposed methodology with some numerical examples. 


\section{Capítulo 1}

\section{Introdução}

\subsection{Apresentação do problema}

$\mathrm{O}$ uso de modelos estatísticos com pontos de mudança têm sido frequente na análise de dados em várias áreas como medicina, epidemiologia, veterinária, controle de qualidade de produtos industriais, controle de acidentes de trabalho, linguística etc. Os pesquisadores dessas áreas geralmente obtém dados ordenados cronológicamente $\mathrm{e}$ procuram identificar se há mudança no comportamento de uma função de interesse básico para seu estudo. A identificação do número de pontos de mudança, a natureza da mudança, ou seja, se ocorre de forma contínua ou com saltos, a localização desses pontos de mudança e o cálculo da magnitude dessas mudanças são aspectos fundamentais na análise desse tipo de dados.

Nesta dissertação consideramos vários modelos com descontinuidade nos pontos de mudança. As estimativas da localização dos pontos e magnitude das mudanças foram obtidas por máxima verossimilhança e por métodos Bayesianos usando aproximações de Laplace e também com simulação de Monte Carlo em cadeias de Markov (MCMC). Esses modelos foram aplicados à conjuntos de dados reais encontrados na literatura e a abordagem Bayesiana tornou viável a inferência em casos onde a aplicação da teoria assintótica seria impraticável. 


\subsection{Revisão bibliográfica}

A determinação do número de pontos de mudança em uma sequência de variáveis aleatórias é a primeira dificuldade do problema. Entre os métodos propostos recentemente, podemos citar o trabalho apresentado por Green (1995) com um método suficientemente geral para ser aplicado à diversos tipos de problemas tais como seleção de variáveis em um modelo de regressão, modelos de misturas com um número desconhecido de componentes, escolha Bayesiana de modelos com diferentes números de parâmetros e também problemas com vários pontos de mudança. Nesse caso, o número de pontos de mudança é um parâmetro do modelo a ser estimado. A implementação desse método não apresenta vantagens quando o número de pontos de mudança pode ser facilmente identificado graficamente. Além disso, pode ser usado um critério Bayesiano de seleção de modelos quando há evidência de que o número de pontos de mudança é pequeno para o conjunto de dados analisado. Uma outra proposta é um método não paramétrico apresentado por Lee (1996), com o qual o autor obteve estimadores consistentes em probabilidade para o número de pontos de mudança.

Outro aspecto importante é determinar se as mudanças nos modelos ocorrem de forma contínua ou com descontinuidade. Há vários trabalhos onde a mudança não acarreta descontinuidade no modelo, por exemplo Stephens (1994) e West e Ogden (1997). Nesta dissertação consideramos apenas os modelos com saltos. Vários problemas desse tipo são encontrados na literatura em diversas áreas.

Uma aplicação na área epidemiológica feita por Henderson e Matthews (1993), apresenta um modelo para o número anual de casos de sindrome hemolítica-uremica como um processo de Poisson com mudança na taxa de ocorrência da doença. Na área de veterinária, com o objetivo de controlar a qualidade da carne suína, temos um estudo apresentado por Christensen e Rudemo (1996), sobre as mudanças na taxa de incidência de uma determinada doença em porcos na Dinarmarca.

Um outro tipo de problema interessante, na área de linguística (filologia), estuda a mudança na taxa de ocorrência de dois tipos de pronomes em 13 manuscritos medievais ordenados cronologicamente, onde um dos interesses é identificar a autoria dos textos. Esse problema foi abordado por Smith (1980) e uma análise Bayesiana foi feita por Stephens (1994) usando um conjunto de dados conhecido como "Lindisfarne scribes data".

Podemos citar ainda o trabalho apresentado por Lee (1998) no qual encontramos a análise Bayesiana de vários outros problemas que envolvem pontos de mudança. Esse 
trabalho mostra duas aplicações onde os dados assumem distribuição normal com mudança na média e variância comum: A primeira analisa os dados do volume anual defluente na barragem de Aswan, no rio Nilo [dados em Cobb, 1978] e a segunda estuda o número anual de acidentes fatais no trânsito de Illinois [dados em Lee e Heghinian, 1977]. Neste mesmo trabalho, foi apresentada uma análise dos dados de acidentes fatais em minas de carvão na Inglaterra [dados em Jarrett, 1979]; nesse caso os intervalos de tempo entre os acidentes sucessivos são considerados independentes e exponencialmente distribuidos assumindo que há uma mudança no tempo médio entre acidentes. Os dados de Jarrett também foram analisados por Raftery e Akman (1986) assumindo densidades a priori conjugadas. Carlin, Gelfand e Smith (1992) converteram esses dados para número anual de acidentes, modelados como um processo de Poisson e formularam um modelo hierárquico. Esse problema foi analisado nessa dissertação considerando os intervalos de tempo entre acidentes, supondo um modelo exponencial com dois pontos de mudança e os resultados foram comparados com os obtidos em Raftery e Akman (1986) e Carlin et al (1992).

Em muitos estudos da área médica o objetivo dos pesquisadores é fazer a comparação de tratamentos novos com os tratamentos tradicionalmente usados para cura ou controle de uma doença. Outro interesse básico destes estudos pode ser comparar grupos de pacientes com características diferentes que recebem o mesmo tratamento, por exemplo, testar se a idade ou sexo dos pacientes podem ser fatores de risco para essa doença. Para responder a essas questões, são usados modelos de sobrevivência (ver por exemplo Lawless, 1982) para os tempos de sobrevida dos pacientes, após administração de um ou mais tratamentos. Esses modelos são representados através de uma função de risco (força de mortalidade), que especifica a distribuição dos dados, facilitando a interpretação dos resultados já que uma mudança nesta função pode indicar o efeito do tratamento ou de outra covariável.

Um estudo sobre tratamento de leucemia não linfoblástica aguda foi apresentado por Matthews e Farewell (1982), onde foi considerado um modelo de sobrevivência exponencial para os tempos de sobrevida dos pacientes. Uma análise Bayesiana desses dados foi apresentada por Achcar e Bolfarine (1989) considerando densidades a priori não informativas para os parâmetros. Uma abordagem Bayesiana assumindo densidades a priori informativas é dada por Achcar e Loibel (1998) utilizando simulação de Monte Carlo em cadeias de Markov. Um modelo com risco variável no tempo foi introduzido por Achcar, Andrade e Loibel (1998). Nesta dissertação, este mesmo conjunto de dados foi utilizado para uma aplicação dos modelos de sobrevivência propostos. 


\subsection{Conteúdo da dissertação}

Esta dissertação está organizado em cinco capitulos. No capítulo 2 apresentamos modelos de sobrevivência com função de risco constante por partes assumindo a existência de um único ponto de mudança. No primeiro modelo proposto assumimos a ocorrência de censura nos dados de sobrevida dos pacientes submetidos a um tratamento. Em seguida apresentamos um modelo para comparação de dois tratamentos para dados sem censura. Em ambos os casos, foram obtidos estimadores de máxima verossimilhança e respectivos intervalos de confiança assintóticos para os parâmetros. A abordagem Bayesiana desses modelos foi apresentada assumindo densidades a priori informativas e não informativas para os parâmetros. Apresentamos os estimadores a posteriori, quando possível, usando o método de aproximação de integrais de Laplace e as densidades condicionais para o uso dos algoritmos de simulação de Monte Carlo em cadeias de Markov.

No capítulo 3 consideramos um modelo mais realista para os dados assumindo uma distribuição Weibull para os tempos de vida, levando a uma função de risco que varia no tempo. Com esse modelo podemos avaliar o efeito do tratamento através da mudança na função de risco com maior precisão. Apresentamos os estimadores de máxima verossimilhança para os parâmetros do modelo. A abordagem Bayesiana assumindo densidades a priori não informativas para os parâmetros permitiu deduzir as densidades condicionais para o uso dos algoritmos de simulação de Monte Carlo em cadeias de Markov.

No capítulo 4 apresentamos a análise Bayesiana de um processo de Poisson com mudança na taxa de ocorrència assumindo densidades a priori não informativas. Neste caso o número de pontos de mudança pode ser determinado graficamente. Apresentamos dois modelos sendo um deles assumindo que existe um único ponto de mudança e outro no qual adotamos dois pontos de mudança. Apresentamos uma abordagem alternativa proposta por Carlin, Gelfand e Smith (1992) para o caso em que há um único ponto de mudança considerando um modelo hierárquico com densidades a priori informativas para os parâmetros.

Algumas aplicações dos modelos apresentados nos capítulos 2, 3 e 4 estão no capítulo 5. Para o modelo com função de risco constante com um tratamento, utilizamos os dados de Matthews e Farewell (1982) para obter e comparar as estimativas de máxima verossimilhança e as estimativas Bayesianas obtidas por aproximação de Laplace e por simulação de MCMC. Esse mesmo tipo de análise e comparação foi feito com um conjunto de dados gerados para o modelo de risco constante com um ponto de mudança e dois 
tratamentos. Para o modelo Weibull apresentamos estimativas de máxima verossimilhança e estimativas Bayesianas obtidas por simulação de MCMC para os dados de Matthews e Farewell (1982). Para a escolha do modelo mais adequado à esses dados usamos o critério Bayesiano baseado nas densidades preditivas ordenadas.

Na segunda parte do capitulo 5 apresentamos aplicação dos modelos desenvolvidos no capítulo 4 para os dados de número de acidentes fatais em minas de carvão na Inglaterra [dados em Jarrett, 1979]. A análise gráfica indica a presença de dois pontos de mudança na taxa de ocorrência. As estimativas a posteriori foram obtidas por simulação de MCMC e os resultados foram comparados com os apresentados por Raftery e Akman (1986). Neste capítulo também apresentamos os resultados do critério Bayesiano para seleção entre os modelos com um ou dois pontos de mudança. 


\section{Capítulo 2}

\section{Modelos de sobrevivência com função de risco constante}

\subsection{Apresentação dos modelos:}

Uma das distribuições de probabilidade mais usadas para dados de tempo de sobrevida é a distribuição exponencial, que pressupõe uma taxa risco constante no tempo. Nesse caso, o interesse é saber se uma taxa de risco constante muda após um determinado periodo, o que indicaria o possível efeito de um tratamento ou de outra covariável como sexo, idade etc. Além disso, é importante saber o instante em que ocorre esta mudança, para avaliar a eficácia ou não de uma nova terapia ou, no caso de outra covariável, decidir se esta representa um fator de risco para a doença estudada ou não, ou seja, caracterizar melhor os fatores de risco.

Em análise de sobrevivência a ocorrência de censuras (observação parcial da resposta) é muito comum devido à interrupção do acompanhamento de uma parte dos pacientes. Esse fato deve ser levado em conta na construção dos modelos afim de se obter resultados precisos. Nesse trabalho, consideramos experimentos onde o prazo para o término $\left(\mathrm{t}_{\mathrm{f}}\right)$ é estipulado pelo pesquisador e os pacientes não entram no estudo necessariamente no início $\left(\mathrm{t}_{\mathrm{o}}\right)$. Esse esquema, denominado de censura tipo I progressiva, produz dados do tipo $T_{i}=\min \left\{T_{i}^{0}, C_{i}\right\}$, sendo $T_{i}^{0}$ os tempos de vida verdadeiros e $C_{i}$ os tempos de censura fixos associados a cada paciente, $i=1, \ldots, n$. Na figura abaixo, temos um exemplo de esquema de censuras tipo I :

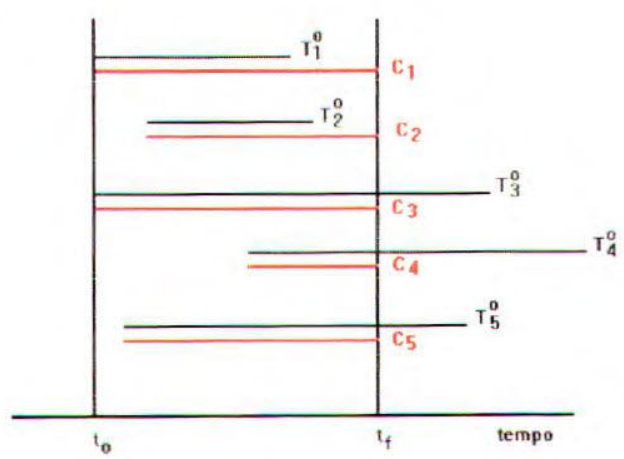




\subsubsection{Modelo Exponencial com um ponto de mudança - Um tratamento}

Considerar o caso em que se quer estimar a função de risco e o ponto de mudança, para um grupo de pacientes que receberam um só tratamento, isto é, sem considerar covariáveis no modelo.

Seja $T \geq 0$, o tempo de sobrevida de um paciente com função de risco definida por:

$\lambda(t)= \begin{cases}\lambda & \text { se } t<\tau \\ \rho \lambda & \text { se } t \geq \tau\end{cases}$

A função densidade de probabilidade de $T$ é dada por:

$$
f(t)= \begin{cases}\lambda e^{-\lambda t} & \text { se } t<\tau \\ \rho \lambda e^{-\lambda \tau-\rho \lambda(t-\tau)} & \text { se } t \geq \tau\end{cases}
$$

A função de sobrevivência é dada por:

$$
S(t)= \begin{cases}e^{-\lambda t} & \text { se } t<\tau \\ e^{-\lambda \tau-\rho \lambda(t-\tau)} & \text { se } t \geq \tau\end{cases}
$$

Os parâmetros $\lambda>0, \quad \rho>0 \quad$ e $\tau>0$ representam a taxa de risco do paciente, a taxa de mudança do risco e o instante da mudança do risco, respectivamente.

\subsubsection{Modelo exponencial com um ponto de mudança - Dois tratamentos}

Considere o caso em que se quer comparar dois tratamentos diferentes (ou dois grupos de pacientes com características diferentes). Seja $T \geq 0$, o tempo de sobrevida de um paciente.

Neste modelo, introduzimos a covariável tratamento, denotada por $X$, onde

$$
X=\left\{\begin{array}{lll}
0 & \text { se } i=1 & \text { (Tratamento 1) } \\
1 & \text { se } i=2 & \text { (Tratamento 2) }
\end{array}\right.
$$


A função de risco neste caso é dada por:

$$
\lambda_{i}(t)=\left\{\begin{array}{ll}
\lambda & \text { se } t<\tau_{i} \\
\rho \lambda e^{\beta x} & \text { se } t \geq \tau_{i}
\end{array} \quad i=1,2\right.
$$

A função densidade de probabilidade é dada por:

$$
f_{i}(t)= \begin{cases}\lambda e^{-\lambda t} & \text { se } t<\tau_{i} \\ \rho \lambda \exp \left\{\beta x-\lambda \tau_{i}-\rho \lambda e^{\beta x}\left(t-\tau_{i}\right)\right\} & \text { se } t \geq \tau_{i}\end{cases}
$$

A função de sobrevivência é dada por:

$$
S_{i}(t)= \begin{cases}e^{-\lambda t} & \text { se } t<\tau_{i} \\ \exp \left\{-\lambda \tau_{i}-\rho \lambda e^{\beta x}\left(t-\tau_{i}\right)\right\} & \text { se } t \geq \tau_{i}\end{cases}
$$

onde $\lambda>0$, representa a taxa de risco do paciente, $\rho>0$ é a taxa de mudança do risco, $\tau_{i}>0(i=1,2)$ são os instantes da mudança do risco para cada tratamento $\mathrm{e}$

$-\infty<\beta<\infty$ é o parâmetro associado à covariável $X$.

Esse modelo já foi abordado anteriormente por Matthews e Farewell (1982) e Matthews, Farewell e Pyke (1985), num estudo de novos medicamentos para o tratamento de leucemia não linfoblástica aguda, considerando um conjunto de dados com censuras. Uma análise Bayesiana desse modelo, com os dados de Matthews e Farewell, foi proposta por Achcar e Bolfarine (1989) e por Ghosh, Joshi e Mukhopadhyay (1993), usando densidades a priori não-informativas, calculadas pela regra de Jeffreys [Box,G.E. e Tiao,G.C., 1973]. Neste estudo, estão apresentadas as densidades a posteriori marginais para os parâmetros do modelo exponencial com um ponto de mudança, com dados sem censuras como também com censuras tipo I e tipo II.

\subsection{Inferência clássica}

Assumindo os modelos da seção anterior, as inferências usuais baseadas em resultados assintóticos dos estimadores de máxima verossimilhança não são facilmente obtidas. Para simplificar essa análise, em alguns casos podemos assumir que o ponto de mudança da taxa de risco seja conhecido. 


\subsubsection{Análise para o modelo exponencial com um tratamento}

Para esse modelo, considerando dados com censuras do tipo I, podemos estimar graficamente o ponto de mudança da taxa de risco $(\tau)$ e calcular os estimadores de máxima verossimilhança e intervalos de confiança para os outros parâmetros $(\lambda$ e $\rho)$.

Para estimar $\tau$ graficamente, observamos a relação entre a função de risco e a função de sobrevivência:

Por definição,

$$
S(t)=P[T>t]=1-F_{T}(t) \quad \text { e } \quad h(t)=\frac{f(t)}{S(t)}
$$

então $\quad f(t)=-S^{\prime}(t) \quad$ e $\quad h(t)=\frac{-S^{\prime}(t)}{S(t)}=-\frac{d \log S(t)}{d t}$

A função de risco acumulada [Ver por exemplo, Cox,D.R. e Oakes,D., 1984] é dada por:

$H(t)=-\log S(t)$

No caso da distribuição exponencial com um ponto de mudança temos,

$$
H(t)= \begin{cases}\lambda t & \text { se } t<\tau \\ \rho \lambda\left[t-\tau\left(\frac{\rho-1}{\rho}\right)\right] & \text { se } t \geq \tau\end{cases}
$$

Portanto, observando o gráfico de $\widehat{H}(t) \times t$, podemos ter uma estimativa aproximada do ponto de mudança, onde $\widehat{H}_{T}(t)=-\log \widehat{S}_{T}(t)$ e $\widehat{S}_{T}(t)$ é o estimador produto limite de Kaplan-Meier para função de sobrevivência [Kaplan, E. L. e Meier, P. , 1958].

Com a estimativa gráfica do ponto de mudança, vamos considerar $L$ como função apenás de $\lambda$ e $\rho$. Como os tempos observados são dados por $T_{i}=\min \left\{T_{i}^{0}, C_{i}\right\}$, definimos uma variável indicadora $\delta$, tal que

$$
\delta_{i}=\left\{\begin{array}{llr}
1 & \text { se } & T_{i}=T_{i}^{0} \\
0 & \text { se } & T_{i}<T_{i}^{0}
\end{array} \quad \quad i=1, \ldots, n\right.
$$


$O$ instante em que ocorre a mudança no risco $(\tau)$, divide o conjunto de dados em duas partes: Uma parte com os tempos de sobrevida menores do que o ponto de mudança e outra com os tempos maiores.Para indicar isto, definimos a variaivel $\epsilon$, tal que

$$
\epsilon_{i}=\left\{\begin{array}{lll}
1 & \text { se } & T_{i}<\tau \\
0 & \text { se } & T_{i} \geq \tau
\end{array} \quad i=1, \ldots, n\right.
$$

Assim a função de verossimilhança para $\lambda, \rho$ e $\tau$ é dada por,

$$
\begin{aligned}
L(\lambda, \rho, \tau)= & \prod_{i=1}^{n}\left\{\left(\lambda e^{-\lambda t_{i}}\right)^{\epsilon_{i}}\left[\rho \lambda \exp \left\{-\lambda \tau-\rho \lambda\left(t_{i}-\tau\right)\right\}\right]^{1-\epsilon_{i}}\right\}^{\delta_{i}} . \\
& \prod_{i=1}^{n}\left\{\left(e^{-\lambda t_{i}}\right)^{\epsilon_{i}}\left[\exp \left\{-\lambda \tau-\rho \lambda\left(t_{i}-\tau\right)\right\}\right]^{1-\epsilon_{i}}\right\}^{1-\delta_{i}}
\end{aligned}
$$

ou

$$
\begin{aligned}
L(\lambda, \rho, \tau) & =\lambda^{d_{3}} \rho^{d_{3}-d_{1}(\tau)} \exp \left\{-\lambda w_{1}(\tau)-\rho \lambda w_{2}(\tau)+\lambda \tau(\rho-1) w_{3}(\tau)\right\}= \\
& =\lambda^{d_{3}} \rho^{d_{3}-d_{1}(\tau)} \exp \left\{-\lambda\left[S_{1}(\tau)+\rho S_{2}(\tau)\right]\right\}
\end{aligned}
$$

onde $\quad d_{1}(\tau)=\sum_{i=1}^{n} \delta_{i} \epsilon_{i} \quad, \quad d_{\underline{2}}(\tau)=\sum_{i=1}^{n} \epsilon_{i} \quad, \quad d_{3}=\sum_{i=1}^{n} \delta_{i}$

$$
w_{1}(\tau)=\sum_{i=1}^{n} \delta_{i} \epsilon_{i} t_{i}+\sum_{i=1}^{n}\left(1-\delta_{i}\right) \epsilon_{i} t_{i}
$$$$
w_{2}(\tau)=\sum_{i=1}^{n} \delta_{i}\left(1-\epsilon_{i}\right) t_{i}+\sum_{i=1}^{n}\left(1-\delta_{i}\right)\left(1-\epsilon_{i}\right) t_{i}
$$$$
w_{3}(\tau)=\sum_{i=1}^{n} \delta_{;}\left(1-\epsilon_{i}\right)+\sum_{i=1}^{n}\left(1-\delta_{i}\right)\left(1-\epsilon_{i}\right)=\sum_{i=1}^{n}\left(1-\epsilon_{i}\right)=n-d_{2}(\tau)
$$$$
S_{1}(\tau)=w_{1}(\tau)+\tau w_{3}(\tau)
$$$$
S_{2}(\tau)=w_{2}(\tau)-\tau w_{3}(\tau)
$$

Assumindo $\tau=\tau^{*}$ conhecido, podemos calcular os estimadores de máxima verossimilhança para $\lambda$ e $\rho$. 
Seja

$$
l\left(\lambda, \rho, \tau^{*}\right)=\ln [L]=d_{3} \ln (\lambda)+\left[d_{3}-d_{1}\left(\tau^{*}\right)\right] \ln (\rho)-\lambda\left[S_{1}\left(\tau^{*}\right)+\rho S_{2}\left(\tau^{*}\right)\right]
$$

Maximizando $l\left(\lambda, \rho, \tau^{*}\right)$, temos

$$
\hat{\lambda}=\frac{d_{1}\left(\tau^{*}\right)}{S_{1}\left(\tau^{*}\right)} \quad \text { e } \quad \hat{\rho}=\frac{S_{1}\left(\tau^{*}\right)\left[d_{3}-d_{1}\left(\tau^{*}\right)\right]}{S_{2}\left(\tau^{*}\right) d_{1}\left(\tau^{*}\right)}
$$

Para o cálculo de intervalos de confiança, assumir a normalidade assintótica dos estimadores de máxima verossimilhança [ver por exemplo, Cox, D.R.e Hinkley, D. 1974]:

$$
\begin{aligned}
& \widehat{\lambda} \stackrel{\mathrm{a}}{\sim} N\left[\lambda, I_{11}^{-1}(\widehat{\lambda})\right] \\
& \widehat{\rho} \stackrel{\mathrm{a}}{\sim} N\left[\rho, I_{22}^{-1}(\widehat{\rho})\right]
\end{aligned}
$$

onde $I$ é a matriz de informação de Fisher esperada dada por:

$$
I(\lambda, \rho)=E\left[\begin{array}{cc}
-\frac{\partial^{2} l(\lambda, \rho)}{\partial \lambda^{2}} & -\frac{\partial^{2} l(\lambda, \rho)}{\partial \lambda \partial \rho} \\
-\frac{\partial^{2} l(\lambda, \rho)}{\partial \rho \partial \lambda} & -\frac{\partial^{2} l(\lambda, \rho)}{\partial \rho^{2}}
\end{array}\right]=\left[\begin{array}{cc}
\frac{n}{\lambda^{2}} & \frac{n e^{-\lambda \tau^{*}}}{\rho \lambda} \\
\frac{n e^{-\lambda \tau^{*}}}{\rho \lambda} & \frac{n e^{-\lambda \tau^{*}}}{\rho^{2}}
\end{array}\right]
$$

Daí, $\operatorname{det} I(\lambda, \rho)=\frac{n^{2} \tau^{*}}{\lambda \rho^{2}}$

A inversa da matriz de informação de Fisher é dada por,

$$
I^{-1}(\lambda, \rho)=\left[\begin{array}{cc}
\frac{\lambda e^{-\lambda \tau^{*}}}{n \tau^{*}} & -\frac{\rho e^{-\lambda \tau^{*}}}{n \tau^{*}} \\
-\frac{\rho e^{-\lambda \tau^{*}}}{n \tau^{*}} & \frac{\rho^{2}}{n \lambda \tau^{*}}
\end{array}\right]
$$

Intervalos de confiança para $\lambda$ e $\rho$ são dados por,

Para $\lambda: \quad \widehat{\lambda} \pm z_{\alpha}\left[\frac{\widehat{\lambda} e^{-\hat{\lambda} \tau^{*}}}{n \tau^{*}}\right]^{1 / 2}$

Para $\rho: \quad \widehat{\rho} \pm \tilde{\sim}_{, x}\left[\frac{\widehat{\rho}^{2}}{n \widehat{\lambda} \tau^{*}}\right]^{1 / 2}$ 
Poderiamos obter os estimativas de máxima verossimilhança para $\lambda, \rho$ e $\tau$ considerando que $\tau$ assume os valores da amostra, ou seja $\tau_{i}=t_{i}$. Encontramos todos os valores $\widehat{\lambda}_{\tau_{i}}$ e $\hat{\rho}_{T_{i}}$ por $(2.9$ ), daí observamos quais são os valores que maximizam $L\left(\widehat{\lambda}_{\tau}, \widehat{\rho}_{\tau}, \tau\right)$.

\subsubsection{Análise para o modelo exponencial com dois tratamentos}

Para este modelo, a estimação dos pontos de mudança para cada tratamento $\left(\tau_{1}\right.$ e $\tau_{2}$ ) também pode ser feita graficamente e com isso podemos obter os estimadores de máxima verossimilhança e intervalos de confiança para os outros parâmetros $(\lambda, \rho$ e $\beta)$. Assim como no modelo com um tratamento, definimos duas variáveis indicadoras; $\delta \mathrm{e} \epsilon$, tal que

$$
\begin{aligned}
& \delta_{i \mathrm{j}}=\left\{\begin{array}{lll}
1 & \text { se } & T_{i j}=T_{i j}^{0} \\
0 & \text { se } & T_{i j}<T_{i j}^{0}
\end{array} \quad i=1,2 \text { e } \quad j=1, \ldots, n_{i}\right. \\
& \epsilon_{\mathrm{i} j}=\left\{\begin{array}{lll}
1 & \text { se } & T_{i j}<\tau_{i} \\
0 & \text { se } & T_{i j} \geq \tau_{i}
\end{array} \quad i=1,2 \text { e } \quad j=1, \ldots, n_{i}\right.
\end{aligned}
$$

A função de verossimilhança nesse caso é dada por,

$$
\begin{aligned}
L\left(\lambda, \rho, \beta, \tau_{1}, \tau_{2}\right)= & \prod_{j=1}^{n_{1}}\left\{\left(\lambda e^{-\lambda t_{1 j}}\right)^{\epsilon_{1 j}}\left[\rho \lambda \exp \left\{-\lambda \tau_{1}-\rho \lambda\left(t_{1 j}-\tau_{1}\right)\right\}\right]^{1-\epsilon_{1 j}}\right\}^{\delta_{1 j}} \\
& \prod_{j=1}^{n_{1}}\left\{\left(e^{-\lambda t_{1 j}}\right)^{\epsilon_{1 j}}\left[\exp \left\{-\lambda \tau_{1}-\rho \lambda\left(t_{1 j}-\tau_{1}\right)\right\}\right]^{1-\epsilon_{1 j}}\right\}^{1-\delta_{1 j}} \\
& \prod_{j=1}^{n_{3}}\left\{\left(\lambda e^{-\lambda t_{2 j}}\right)^{\epsilon_{2 j}}\left[\rho \lambda e^{\beta} \exp \left\{-\lambda \tau_{2}-\rho \lambda e^{\beta}\left(t_{2 j}-\tau_{2}\right)\right\}\right]^{1-\epsilon_{2 j}}\right\}^{\delta_{2 j}} \\
& \prod_{j=1}^{n_{2}}\left\{\left(e^{-\lambda t_{2 j}}\right)^{\epsilon_{2 j}}\left[\exp \left\{-\lambda \tau_{2}-\rho \lambda e^{\beta}\left(t_{2 j}-\tau_{2}\right)\right\}\right]^{1-\epsilon_{2 j}}\right\}^{1-\delta_{2 j}}
\end{aligned}
$$

ou

$$
\begin{aligned}
& L\left(\lambda, \rho, \beta, \tau_{1}, \tau_{2}\right)= \\
& =\lambda^{d_{3}} \rho^{d_{3}-d_{1}\left(\tau_{1}, \tau_{2}\right)} e^{\left\{\beta\left(d_{3}^{(2)}-d_{1}^{(2)}\right)-\lambda\left[S_{1}\left(\tau_{1}, \tau_{2}\right)+\rho S_{2}\left(\tau_{1}, \tau_{2}, \beta\right)\right]\right\}}
\end{aligned}
$$


onde

$$
\begin{aligned}
& d_{1}^{(i)}\left(\tau_{i}\right)=\sum_{j=1}^{n_{i}} \delta_{i j} \epsilon_{i j}, \quad \text { e } \quad d_{1}\left(\tau_{1}, \tau_{2}\right)=d_{1}^{(1)}\left(\tau_{1}\right)+d_{1}^{(2)}\left(\tau_{2}\right) \\
& d_{2}^{(i)}\left(\tau_{i}\right)=\sum_{j=1}^{n_{i}} \epsilon_{i j}, \\
& d_{3}^{(i)}=\sum_{j=1}^{n_{i}} \delta_{i j}, \quad \text { e } \quad d_{3}=d_{3}^{(1)}+d_{3}^{(2)} \\
& w_{1}^{(i)}\left(\tau_{i}\right)=\sum_{j=1}^{n_{1}} \delta_{i j} \epsilon_{i j} t_{i j}+\sum_{j=1}^{n_{1}}\left(1-\delta_{i j}\right) \epsilon_{i j} t_{i j} \\
& w_{2}^{(i)}\left(\tau_{i}\right)=\sum_{j=1}^{n_{2}} \delta_{i j}\left(1-\epsilon_{i j}\right) t_{i j}+\sum_{j=1}^{n_{2}}\left(1-\delta_{i j}\right)\left(1-\epsilon_{i j}\right) t_{i j} \\
& i=1,2 . \\
& S_{1}\left(\tau_{1}, \tau_{2}\right)=w_{1}^{(1)}\left(\tau_{1}\right)+w_{1}^{(2)}\left(\tau_{2}\right)+\tau_{1}\left(n_{1}-d_{2}^{(1)}\right)+\tau_{2}\left(n_{2}-d_{2}^{(2)}\right) \\
& S_{2}\left(\tau_{1}, \tau_{2}, \beta\right)=w_{2}^{(1)}\left(\tau_{1}\right)-\tau_{1}\left(n_{1}-d_{2}^{(1)}\right)+e^{\beta}\left(w_{2}^{(2)}\left(\tau_{2}\right)-\tau_{2}\left(n_{2}-d_{2}^{(2)}\right)\right)
\end{aligned}
$$

O logaritmo da função de verossimilhança assumindo $\tau_{1}=\tau_{1}^{*}$ e $\tau_{2}=\tau_{2}^{*}$ conhecidos, é dado por,

$$
\begin{aligned}
l\left(\lambda, \rho, \beta, \tau_{1}^{*}, \tau_{2}^{*}\right)= & \ln \left[L\left(\lambda, \rho, \beta, \tau_{1}^{*}, \tau_{2}^{*}\right)\right]=d_{3} \ln (\lambda)+\left[d_{3}-d_{1}\left(\tau_{1}^{*}, \tau_{2}^{*}\right)\right] \ln (\rho) \\
& +\beta\left[d_{3}^{(2)}-d_{1}^{(2)}\left(\tau_{2}^{*}\right)\right]-\lambda\left[S_{1}\left(\tau_{1}^{*}, \tau_{2}^{*}\right)+\rho S_{2}\left(\tau_{1}^{*}, \tau_{2}^{*}, \beta\right)\right]
\end{aligned}
$$

Maximizando $l\left(\lambda, \rho, \beta, \tau_{1}^{*}, \tau_{2}^{*}\right)$, temos

$$
\begin{aligned}
& \widehat{\lambda}=\frac{d_{1}\left(\tau_{1}^{*}, \tau_{2}^{*}\right)}{S_{1}\left(\tau_{1}^{*}, \tau_{2}^{*}\right)}, \\
& \widehat{\rho}=\frac{\left[d_{3}-d_{1}\left(\tau_{1}^{*}, \tau_{2}^{*}\right)\right] S_{1}\left(\tau_{1}^{*}, \tau_{2}^{*}\right)}{d_{1}\left(\tau_{1}^{*}, \tau_{2}^{*}\right) S_{2}\left(\tau_{1}^{*}, \tau_{2}^{*}, \beta\right)}
\end{aligned}
$$




$$
\begin{aligned}
& e^{\widehat{\beta}}=\frac{\left[d_{3}^{(2)}-d_{1}^{(2)}\left(\tau_{2}^{*}\right)\right]\left[w_{2}^{(1)}\left(\tau_{1}^{*}\right)-\tau_{1}^{*}\left(n_{1}-d_{2}^{(1)}\right)\right]}{\left[d_{3}^{(1,}-d_{1}^{(1)}\left(\tau_{1}^{*}\right)\right]\left[w_{2}^{(2)}\left(\tau_{1}^{*}\right)-\tau_{2}^{*}\left(n_{2}-d_{2}^{(2)}\right)\right]}, \\
& \widehat{\beta}=\ln \left[\frac{\left[d_{3}^{(2)}-d_{1}^{(2)}\left(\tau_{2}^{*}\right)\right]\left[w_{2}^{\prime 1)}\left(\tau_{1}^{*}\right)-\tau_{1}^{*}\left(n_{1}-d_{2}^{(1)}\right)\right]}{\left[d_{3}^{(1)}-d_{1}^{(1)}\left(\tau_{1}^{*}\right)\right]\left[w_{2}^{(2)}\left(\tau_{1}^{*}\right)-\tau_{2}^{*}\left(n_{2}-d_{2}^{(2)}\right)\right]}\right]
\end{aligned}
$$

Intervalos de confiança para $\lambda, \rho$ e $\beta$ podem ser obtidos a partir das distribuições normais limite para $\widehat{\lambda}, \widehat{\rho}$ e $\widehat{\beta}$.

Neste caso, as variâncias podem ser calculadas com a inversa da matriz de informação de Fisher observada $\left(I_{0}\right)$ devido à dificuldade de obter a matriz de informação de Fisher esperada,

$\widehat{\lambda} \stackrel{a}{\sim} N\left[\lambda, I_{0}^{-1}(\widehat{\lambda})\right]$

$\widehat{\rho} \stackrel{\mathrm{a}}{\sim} N\left[\rho, I_{0}^{-1}(\widehat{\rho})\right]$

$\widehat{\beta} \stackrel{a}{\sim} N\left[\beta, I_{0}^{-1}(\widehat{\beta})\right]$

A matriz de informação de Fisher observada é dada por,

$$
I_{o}=\left[\begin{array}{ccc}
\frac{d_{3}}{\lambda^{2}} & S_{2}\left(\tau_{1}^{*}, \tau_{2}^{*}, \beta\right) & \rho D\left(\tau_{1}^{*}, \tau_{2}^{*}, \beta\right) \\
S_{2}\left(\tau_{1}^{*}, \tau_{2}^{*}, \beta\right) & \frac{\left[d_{3}-d_{1}\left(\tau_{1}^{*}, \tau_{2}^{*}\right)\right]}{\rho^{2}} & \lambda D\left(\tau_{1}^{*}, \tau_{2}^{*}, \beta\right) \\
\rho D\left(\tau_{1}^{*}, \tau_{2}^{*}, \beta\right) & \lambda D\left(\tau_{1}^{*}, \tau_{2}^{*}, \beta\right) & \lambda \rho D\left(\tau_{1}^{*}, \tau_{2}^{*}, \beta\right)
\end{array}\right]
$$

onde $\quad \widehat{D}=\widehat{D}\left(\tau_{1}^{*}, \tau_{2}^{*}, \widehat{\beta}\right)=\left[w_{2}^{(2)}\left(\tau_{1}^{*}\right)-\tau_{2}^{*}\left(n_{2}-d_{2}^{(2)}\right)\right] e^{\widehat{\beta}}$, calculado em: $\lambda=\widehat{\lambda}, \rho=\widehat{\rho}, \beta=\widehat{\beta}$ e $D=\widehat{D}$. 
As variâncias dos estimadores são dadas por:

$V(\widehat{\lambda})=\frac{\lambda^{2}\left[d_{1}\left(\tau_{1}^{*}, \tau_{2}^{*}\right)-d_{3}+D \lambda \rho e^{\beta}\right]}{D \lambda \rho e^{\beta}\left[2 d_{3}-2 \lambda \rho S_{2}\left(\tau_{1}^{*}, \tau_{2}^{*}, \beta\right)-d_{1}\left(\tau_{1}^{*}, \tau_{2}^{*}\right)\right]-d_{3}\left[d_{3}-d_{1}\left(\tau_{1}^{*}, \tau_{2}^{*}\right)\right]+\lambda^{2} \rho^{2} S_{2}\left(\tau_{1}^{*}, \tau_{2}^{*}, \beta\right)}$

$V(\hat{\rho})=\frac{\rho^{2}\left[D \lambda \rho e^{\beta}-d_{3}\right]}{D \lambda \rho e^{\beta}\left[2 d_{3}-2 \lambda \rho S_{2}\left(\tau_{1}^{*}, \tau_{2}^{*}, \beta\right)-d_{1}\left(\tau_{1}^{*}, \tau_{2}^{*}\right)\right]-d_{3}\left[d_{3}-\bar{d}_{1}\left(\tau_{1}^{*}, \tau_{2}^{*}\right)\right]+\lambda^{2} \rho^{2} S_{2}\left(\tau_{1}^{*}, \tau_{2}^{*}, \beta\right)}$

$V(\widehat{\beta})=\frac{\left\{\lambda^{2} \rho^{2} S_{2}\left(\tau_{1}^{*}, \tau_{2}^{*}, \beta\right)-d_{3}\left[d_{3}-d_{1}\left(\tau_{1}^{*}, \tau_{2}^{*}\right)\right]\right\} e^{-\beta}}{D^{2} \lambda^{2} \rho^{2}\left\{e^{\beta}\left[2 d_{3}-2 \lambda \rho S_{2}\left(\tau_{1}^{*}, \tau_{2}^{*}, \beta\right)-d_{1}\left(\tau_{1}^{*}, \tau_{2}^{*}\right)\right]-d_{3}\left[d_{3}-d_{1}\left(\tau_{1}^{*}, \tau_{2}^{*}\right)\right]+\lambda^{2} \rho^{2} S_{2}\left(\tau_{1}^{*}, \tau_{2}^{*}, \beta\right)\right\}}$

calculadas em: $\lambda=\widehat{\lambda}, \rho=\widehat{\rho}, \beta=\widehat{\beta}$ e $D=\widehat{D}$

Intervalos de confiança para $\lambda, \rho$ e $\beta$ são dados, repectivamente, por,

$$
\begin{aligned}
& \widehat{\lambda} \pm z_{\alpha}[V(\widehat{\lambda})]^{1 / 2} \\
& \widehat{\rho} \pm z_{\alpha}[V(\widehat{\rho})]^{1 / 2} \\
& \widehat{\beta} \pm z_{\alpha}[V(\widehat{\beta})]^{1 / 2}
\end{aligned}
$$

\subsection{Inferência Bayesiana}

A análise Bayesiana dos modelos com risco constante com um ponto de mudança consiste na obtenção das densidades a posteriori marginais e seus sumários de interesse (como médias e modas a posteriori). Para isso, consideramos densidades a priori nãoinformativas e também podemos incorporar informações de especialistas na forma de densidades a priori informativas.

Nesta seção apresentamos as densidades marginais e médias a posteriori calculadas pelo método de aproximação de integrais de Laplace [ver Tierney,L. e Kadane,J.B., 1986] e também usando métodos de simulação MCMC, ou seja, com os algoritmos de amostrador 
de Gibbs [ver Gelfand,A.E. e Smith,A.F.M., 1990] e Metropolis-Hastings [ver Chib,S. e Greenberg,E., 1995 ou Smith,A.F.M. e Roberts,G.O., 1993]. Essas técnicas estão apresentadas no apêndice.

Quando assumimos os pontos de mudança conhecidos, estamos considerando as estimativas gráficas dadas anteriormente.

\subsubsection{Análise do modelo exponencial com um tratamento}

Uso do método de aproximação de Laplace

Primeiramente assumimos densidades a priori não informativas para os parâmetros $\lambda$ e $\rho$ e ponto de mudança conhecido $\tau=\tau^{*}$. Considerar a função de verossimilhança dada em (2.7):

$$
L\left(\lambda, \rho, \tau^{*}\right)=\lambda^{d_{3}} \rho^{d_{3}-d_{1}\left(\tau^{*}\right)} \exp \left\{-\lambda\left[S_{1}\left(\tau^{*}\right)+\rho S_{2}\left(\tau^{*}\right)\right]\right\}
$$

Assumindo independência a priori dos parâmetros, uma densidade conjunta a priori para $\lambda$ e $\rho$ é dada por:

$\pi_{0}(\lambda, \rho)=\pi_{0}(\lambda) \pi_{0}(\rho)$

O cálculo da densidade conjunta a priori não informativa, segundo a regra de Jeffreys, é feito como segue:

A densidade a priori de Jeffreys para $\lambda$ é dada por:

$$
J(\lambda)=[I(\lambda)]^{1 / 2}, \quad \text { onde } I(\lambda)=-E\left\{\frac{\partial^{2} L(\lambda, \rho)}{\partial \lambda^{2}}\right\}
$$

A densidade a priori de Jeffreys para $\rho$ é dada por:

$$
J(\rho)=[I(\rho)]^{1 / 2}, \quad \text { onde } I(\rho)=-E\left\{\frac{\partial^{2} L(\lambda, \rho)}{\partial \rho^{2}}\right\}
$$

Para esse modelo, usando a regra de Jeffreys, as densidades marginais a priori para $\lambda$ e $\rho$ são, repectivamente,

$$
\pi_{0}(\lambda)=J(\lambda) \propto \frac{1}{\lambda} \quad \text { e } \quad \pi_{0}(\rho)=J(\rho) \propto \frac{1}{\rho}
$$


Portanto, a densidade conjunta a priori, assumindo independencia entre $\lambda$ e $\rho$ é dada por:

$$
\pi_{0}(\lambda, \rho) \propto \frac{1}{\lambda \rho}
$$

A densidade conjunta a posteriori é dada por:

$\pi(\lambda, \rho \backslash \tau, \mathcal{D}) \propto \pi_{0}(\lambda, \rho) L(\lambda, \rho, \tau)$

isto é,

$\pi(\lambda, \rho \backslash \tau, \mathcal{D}) \propto \lambda^{d_{3}-1} \rho^{d_{3}-d_{1}\left(\tau^{*}\right)-1} \exp \left\{-\lambda\left[S_{1}\left(\tau^{*}\right)+\rho S_{2}\left(\tau^{*}\right)\right]\right\}$

A densidade marginal a posteriori para $\lambda$ é dada por:

$\pi(\lambda \backslash \mathcal{D}) \propto \int_{0}^{\infty} \pi(\lambda, \rho \backslash \tau, \mathcal{D}) d \rho$

isto é,

$$
\begin{aligned}
\pi(\lambda \backslash \mathcal{D}) & \propto \lambda^{d_{3}-1} \exp \left\{-\lambda S_{1}\left(\tau^{*}\right)\right\} \int_{0}^{\infty} \rho^{d_{3}-d_{1}\left(\tau^{*}\right)-1} \exp \left\{-\rho \lambda S_{2}\left(\tau^{*}\right)\right\} d \rho \\
& =\lambda^{d_{3}-1} \exp \left\{-\lambda S_{1}\left(\tau^{*}\right)\right\} \frac{\Gamma\left[d_{3}-d_{1}\left(\tau^{*}\right)\right]}{\left[S_{2}\left(\tau^{*}\right)\right]^{d_{3}-d_{1}\left(\tau^{*}\right)} \lambda^{d_{3}-d_{1}\left(\tau^{*}\right)}} \\
& =\frac{\Gamma\left[d_{3}-d_{1}\left(\tau^{*}\right)\right]}{\left[S_{2}\left(\tau^{*}\right)\right]^{d_{3}-d_{1}\left(\tau^{*}\right)}} \lambda^{d_{1}\left(\tau^{*}\right)-1} \exp \left\{-\lambda S_{1}\left(\tau^{*}\right)\right\}
\end{aligned}
$$

assim,

$\left.\pi(\lambda \backslash \mathcal{D}) \propto \lambda^{d_{1} \cdot \tau^{*}}\right)-1 \exp \left\{-\lambda S_{1}\left(\tau^{*}\right)\right\}$, isto é, $\lambda \backslash \mathcal{D} \sim \operatorname{Gama}\left[d_{1}\left(\tau^{*}\right), S_{1}\left(\tau^{*}\right)\right]$ 
A média e a moda a posteriori de $\lambda$ são dadas por:

$$
E(\lambda)=\frac{d_{1}\left(\tau^{*}\right)}{S_{1}\left(\tau^{*}\right)} \quad M o(\lambda)=\frac{d_{1}\left(\tau^{*}\right)-1}{S_{1}\left(\tau^{*}\right)}
$$

A densidade marginal a posteriori de $\rho$ é dada por,

$\pi(\rho \backslash \mathcal{D}) \propto \int_{0}^{\infty} \pi(\lambda, \rho \backslash \tau, \mathcal{D}) d \lambda$

isto é,

$$
\begin{aligned}
\pi(\rho \backslash \mathcal{D}) & \propto \rho^{d_{3}-d_{1}\left(\tau^{*}\right)-1} \int_{0}^{\infty} \lambda^{d_{3}-1} \exp \left\{-\lambda\left[S_{1}\left(\tau^{*}\right)+\rho S_{2}\left(\tau^{*}\right)\right]\right\} d \lambda \\
& =\rho^{d_{3}-d_{1}\left(\tau^{*}\right)-1} \frac{\Gamma\left(d_{3}\right)}{\left[S_{1}\left(\tau^{*}\right)+\rho S_{2}\left(\tau^{*}\right)\right]^{d_{3}}}
\end{aligned}
$$

assim,

$$
\pi(\rho \backslash \mathcal{D}) \propto \frac{\rho^{d_{3}-d_{1}\left(\tau^{*}\right)-1}}{\left[S_{1}\left(\tau^{*}\right)+\rho S_{2}\left(\tau^{*}\right)\right]^{d_{3}}}
$$

Portanto, $\rho \backslash \mathcal{D}$ não segue uma distribuição conhecida e o cálculo da média a posteriori requer o uso do método de aproximação de Laplace. Assim temos:

$E(\rho \backslash \mathcal{D})=\frac{\int \rho \pi(\rho \backslash \mathcal{D}) d \rho}{\int \pi(\rho \backslash \mathcal{D}) d \rho}$

Encontramos uma aproximação à $E(\rho \backslash \mathcal{D})$ com,

$L^{*}(\rho)=-\frac{1}{n}\left[\left[d_{3}-d_{1}\left(\tau^{*}\right)\right] \ln (\rho)-d_{3} \ln \left[S_{1}\left(\tau^{*}\right)+\rho S_{2}\left(\tau^{*}\right)\right]+\ln \left[\Gamma\left(d_{3}\right)\right]\right]$

sendo o máximo de $L^{*}$ dado por.

$m^{*}=\frac{S_{1}\left(\tau^{*}\right)\left[d_{3}-d_{1}\left(\tau^{*}\right)\right]}{S_{2}\left(\tau^{*}\right)\left[d_{1}\left(\tau^{*}\right)+1\right]}$ 


$$
\begin{aligned}
& V^{*}(\rho)=\left[\frac{d_{3}-d_{1}\left(\tau^{*}\right)-1}{n m^{2}}-\frac{d_{3} S_{2}\left(\tau^{*}\right)}{n\left[S_{1}\left(\tau^{*}\right)+m S_{2}\left(\tau^{*}\right)\right]^{2}}\right]^{-1}, \\
& L(\rho)=-\frac{1}{n}\left[\left[d_{3}-d_{1}\left(\tau^{*}\right)-1\right] \ln (\rho)-d_{3} \ln \left[S_{1}\left(\tau^{*}\right)+\rho S_{2}\left(\tau^{*}\right)\right]+\ln \left[\Gamma\left(d_{3}\right)\right]\right] \\
& m=\frac{S_{1}\left(\tau^{*}\right)\left[d_{3}-d_{1}\left(\tau^{*}\right)-1\right]}{S_{2}\left(\tau^{*}\right)\left[d_{1}\left(\tau^{*}\right)+1\right]} \mathrm{e} \\
& V(\rho)=\left[\frac{d_{3}-d_{1}\left(\tau^{*}\right)-1}{n m^{2}}-\frac{d_{3} S_{2}\left(\tau^{*}\right)}{n\left[S_{1}\left(\tau^{*}\right)+m S_{2}\left(\tau^{*}\right)\right]^{2}}\right]^{-1} .
\end{aligned}
$$

As definições de $L, L^{*}, V$ e $V^{*}$ estão no apêndice.

A moda a posteriori de $\rho$ édada por:

$$
M o(\rho)=\frac{S_{1}\left(\tau^{*}\right)\left[d_{3}-d_{1}\left(\tau^{*}\right)-1\right]}{S_{2}\left(\tau^{*}\right)\left[d_{1}\left(\tau^{*}\right)+1\right]}
$$

Agora, considerar a função de verossimilhança dada em (2.7), com $r$ conhecido e assumir as seguintes densidades a priori informativas para $\lambda$ e $\rho$ :

$\lambda \sim \operatorname{Gama}\left[a_{1}, b_{1}\right]$

$\rho \sim \operatorname{Gama}\left[a_{2}, b_{2}\right]$

onde $a_{1}, b_{1}, a_{2}$ e $b_{2}$ são constantes.

Com essas densidades a priori, a densidade conjunta a posteriori é dada por:

$\pi(\lambda, \rho \backslash \tau, \mathcal{D}) \propto$

$\lambda^{d_{3}+a_{1}-1} \rho^{d_{3}-d_{1}\left(\tau^{*}\right)+a_{2}-1} \exp \left\{-\lambda\left[b_{1}+S_{1}\left(\tau^{*}\right)+\rho S_{2}\left(\tau^{*}\right)\right]-\rho b_{2}\right\}$

A densidade marginal a posteriori de $\lambda$ é dada por:

$\pi(\lambda \backslash \mathcal{D}) \propto$

$\propto \lambda^{d_{3}+2_{1}-1} \exp \left\{-\lambda\left[b_{1}+S_{1}\left(\tau^{*}\right)\right]\right\} \int_{0}^{\infty} \rho^{d_{3}-d_{1}\left(\tau^{*}\right)+a_{2}-1} \exp \left\{-\rho\left[\lambda S_{2}\left(\tau^{*}\right)+b_{2}\right]\right\} d \rho$ isto é, 
$\pi(\lambda \backslash \mathcal{D}) \propto \lambda^{d_{3}+a_{1}-1} \exp \left\{-\lambda\left[b_{1}+S_{1}\left(\tau^{*}\right)\right]\right\} \frac{\Gamma\left(d_{3}-d_{1}\left(\tau^{*}\right)+a_{2}\right)}{\left[\lambda S_{2}\left(\tau^{*}\right)+b_{2}\right]^{d_{3}-d_{1}\left(\tau^{*}\right)+a_{2}}}$

assim,

$\pi(\lambda \backslash \mathcal{D}) \propto \frac{\lambda^{d_{3}+a_{1}-1}}{\left[\lambda S_{2}(\tau)+b_{2}\right]^{d_{3}-d_{1}(\tau)+a_{2}}} \exp \left\{-\lambda\left[b_{1}+S_{1}(\tau)\right]\right\}$

Portanto, $\pi(\lambda \mid \mathcal{D})$ não é uma distribuição conhecida e a média a posteriori pode ser obtida pelo método de aproximação de Laplace usando método iterativo de NewtonRaphson

A densidade marginal a posteriori de $\rho$ é obtida como segue:

$\pi(\rho \backslash \mathcal{D}) \propto$

$\dot{\propto} \rho^{d_{3}-d_{1}\left(\tau^{*}\right)+a_{2}-1} \exp \left\{-\rho b_{2}\right\} \int_{0}^{\infty} \lambda^{d_{3}+a_{1}-1} \exp \left\{-\lambda\left[b_{1}+S_{1}\left(\tau^{*}\right)+\rho S_{2}\left(\tau^{*}\right)\right]\right\} d \lambda$

isto é,

$\pi(\rho \backslash \mathcal{D}) \propto \rho^{d_{3}-d_{1}(\tau)+a_{2}-1} \exp \left\{-\rho b_{2}\right\} \frac{\Gamma\left(d_{3}+a_{1}\right)}{\left[b_{1}+S_{1}(\tau)+\rho S_{2}(\tau)\right]^{d_{3}-d_{1}(\tau)}}$

assim,

$\pi(\rho \backslash \mathcal{D}) \propto \frac{\rho^{d_{3}-d_{1}(\tau)+a_{2}-1}}{\left[b_{1}+S_{1}(\tau)+\rho S_{2}(\tau)\right]^{d_{3}+d_{1}(\tau)}} \exp \left\{-\rho b_{2}\right\}$

Notamos que $\pi(\rho \backslash \mathcal{D})$ não é uma distribuição conhecida e o cálculo da média a posteriori de $\rho$ também pode ser feito pelo método de aproximação de Laplace (numericamente). 
Assumindo agora que o ponto de mudança no risco, $\tau$, é desconhecido, escolhemos uma densidade a priori não informativa para $\tau$ dada por $\pi_{0}(\tau)=\frac{1}{n}$. Considerando a densidade conjunta a priori não informativa para $\lambda$ e $\rho$ dada em (2.22), temos que a densidade conjunta a priori, assumindo independência entre os parâmetros, é dada por:

$\pi_{0}(\lambda, \rho, \tau) \propto \frac{1}{\lambda \rho}$

Então, a densidade conjunta a posteriori para $\lambda, \rho$ e $\tau$ é dada por:

$\pi(\lambda, \rho, \tau \backslash \mathcal{D}) \propto \lambda^{d_{3}-1} \rho^{d_{3}-d_{1}(\tau)-1} \exp \left\{-\lambda\left[S_{1}(\tau)+\rho S_{2}(\tau)\right]\right\}$

A densidade marginal a posteriori de $\tau$ é dada por,

$\pi\left(\tau_{i}=t_{i} \backslash \mathcal{D}\right) \propto \int_{0}^{\infty} \int_{0}^{\infty} \pi(\lambda, \rho, \tau \backslash \mathcal{D}) d \rho d \lambda$

Assim,

$\pi\left(\tau_{i}=t_{i} \backslash \mathcal{D}\right) \propto \int_{0}^{\infty} \frac{\Gamma\left[d_{3}-d_{1}(\tau)\right]}{\left[S_{2}(\tau)\right]^{d_{3}-d_{1}(\tau)} \lambda^{d_{3}-d_{1}(\tau)}} \lambda^{d_{3}-1} \exp \left\{-\lambda\left[S_{1}(\tau)\right]\right\} d \lambda$

isto é,

$\pi\left(\tau_{i}=t_{i} \backslash \mathcal{D}\right) \propto \frac{\Gamma\left[d_{3}-d_{1}(\tau)\right] \Gamma\left[d_{1}(\tau)\right]}{\left[S_{2}(\tau)\right]^{d_{3}-d_{1}(\tau)}\left[S_{1}(\tau)\right]^{d_{1}(\tau)}}$

Um estimador Bayesiano de $\tau$ é a moda de $\pi\left(\tau_{i}=t_{i} \backslash \mathcal{D}\right)$. 


\section{Uso de métodos de simulação MCMC}

Como já foi visto, com a priori não informativa de Jeffreys (2.22) com ponto de mudança desconhecido, a densidade conjunta a posteriori para $\lambda, \rho$ e $r$ é dada por,

$\pi(\lambda, \rho, \tau \mathcal{D}) \propto \lambda^{d_{3}-1} \rho^{d_{3}-d_{1}(\tau)-1} \exp \left\{-\lambda\left[S_{1}(\tau)+\rho S_{2}(\tau)\right]\right\}$

onde $S_{1}(\tau)$ e $S_{2}(\tau)$ são dadas em (2.8).

As densidades condicionais a posteriori para o algoritmo amostrador de Gibbs são dadas por,

$\pi(\lambda \backslash \rho, \tau, \mathcal{D}) \propto \lambda^{d_{3}-1} \exp \left\{-\lambda\left[S_{1}(\tau)+\rho S_{2}(\tau)\right]\right\}$

isto é, $\lambda \backslash \rho, \tau, \mathcal{D} \sim \operatorname{Gama}\left[d_{3}, S_{1}(\tau)+\rho S_{2}(\tau)\right]$

$\pi(\rho \backslash \lambda, \tau, \mathcal{D}) \propto \rho^{d_{3}-d_{1}(\tau)-1} \exp \left\{-\rho \lambda S_{2}(\tau)\right\}$

isto é , $\rho \backslash \lambda, \tau, \mathcal{D} \sim \operatorname{Gama}\left[d_{3}-d_{1}(\tau), \lambda S_{2}(\tau)\right]$

e

$\pi(\tau \backslash \lambda, \rho, \mathcal{D}) \propto \rho^{-d_{i}(\tau)} \exp \left\{-\lambda\left[S_{1}(\tau)+\rho S_{2}(\tau)\right]\right\}$

Para $\lambda$ e $\rho$ podemos usar o algoritmo de amostrador de Gibbs para gerar as amostras. No caso do parâmetro $r$, é necessário o uso do algoritmo Metropolis-Hastings, já que a distribuição condicional de $\tau$ dado $\lambda$ e $\rho$ não pode ser gerada diretamente. Para isso usamos como núcleo de transição, uma distribuição Uniforme para $\tau$.

Supor agora $\tau=T^{*}$ e assumir as densidades a priori informativas para $\lambda$ e $\rho$ dadas em (2.28). Com isso a densidade conjunta a posteriori para $\lambda$ e $\rho$ é dada por:

$$
\begin{aligned}
& \pi(\lambda, \rho \backslash \tau, \mathcal{D}) \propto \\
& \propto \lambda^{d_{3}+a_{1}-1} \rho^{d_{3}-d_{1}\left(\tau^{*}\right)+a_{2}-1} \exp \left\{-\lambda\left[b_{1}+S_{1}\left(\tau^{*}\right)+\rho S_{2}\left(\tau^{*}\right)\right]-\rho b_{2}\right\}
\end{aligned}
$$


As densidades condicionais a posteriori para o algoritmo amostrador de Gibbs são dadas por:

$$
\begin{aligned}
& \pi(\lambda \backslash \rho, \tau, \mathcal{D}) \propto \lambda^{d_{3}+a_{1}-1} \exp \left\{-\lambda\left[b_{1}+S_{1}\left(\tau^{*}\right)+\rho S_{2}\left(\tau^{*}\right)\right]\right\} \\
& \text { isto é, } \lambda \backslash \rho, \tau, \mathcal{D} \sim \operatorname{Gama}\left[d_{3}+a_{1}, b_{1}+S_{1}\left(\tau^{*}\right)+\rho S_{2}\left(\tau^{*}\right)\right] \\
& \text { e } \\
& \pi(\rho \backslash \lambda, \tau, \mathcal{D}) \propto \rho^{d_{3}-d_{1}\left(\tau^{*}\right)+a_{2}-1} \exp \left\{-\rho\left[b_{2}+\lambda S_{2}\left(\tau^{*}\right)\right]\right\} \\
& \text { isto é, } \rho \backslash \lambda, \tau, \mathcal{D} \sim \operatorname{Gama}\left[d_{3}-d_{1}\left(\tau^{*}\right)+a_{2}, b_{2}+\lambda S_{2}\left(\tau^{*}\right)\right]
\end{aligned}
$$

\subsubsection{Análise do modelo exponencial com dois tratamentos}

Esse modelo difere do primeiro ao incorporar uma covariável na análise, que neste caso indica tratamento. Consideramos a função de verossimilhança dada em (2.13), assumindo densidades a priori não informativas e os pontos de mudança conhecidos $\tau_{1}=\tau_{1}^{*} \mathrm{e} \tau_{2}=\tau_{2}^{*}$, isto é,

$$
\begin{aligned}
& L\left(\lambda, \rho, \beta, \tau_{1}, \tau_{2}\right)= \\
& =\lambda^{d_{3}} \rho^{d_{3}-d_{1}\left(\tau_{1}^{*}, \tau_{2}^{*}\right)} e^{\left\{\beta\left(d_{3}^{(2)}-d_{1}^{(2)}\right)-\lambda\left[S_{1}\left(\tau_{1}^{*}, \tau_{2}^{*}\right)+\rho S_{2}\left(\tau_{1}^{*}, \tau_{2}^{*}, \beta\right)\right]\right\}}
\end{aligned}
$$

Assumindo independencia a priori nos parâmetros, uma densidade conjunta a priori não informativa é dada por $\pi_{0}(\lambda, \rho, \beta) \propto \frac{1}{\lambda \rho}$.

Com essa escolha de densidade a priori, a densidade conjunta a posteriori é dada por:

$\pi\left(\lambda, \rho, \beta \backslash \tau_{1}, \tau_{2}, \mathcal{D}\right) \propto \pi_{0}(\lambda, \rho, \beta) L\left(\lambda, \rho, \beta, \tau_{1}, \tau_{2}\right)$

isto é,

$$
\begin{aligned}
& \pi\left(\lambda, \rho, \beta \backslash \tau_{1}, \tau_{2}, \mathcal{D}\right) \propto \\
& \propto \lambda^{d_{3}-1} \rho^{d_{3}-d_{1}\left(\tau_{1}^{*}, \tau_{2}^{*}\right)-1} e^{\left\{\beta\left(d_{3}^{(2)}-d_{1}^{(2)}\right)-\lambda\left[S_{1}\left(\tau_{1}^{*}, \tau_{2}^{*}\right)+\rho S_{2}\left(\tau_{1}^{*}, \tau_{2}^{*}, \beta\right)\right]\right\}}
\end{aligned}
$$


A densidade marginal a posteriori de $\lambda$ é dada por:

$$
\begin{aligned}
& \pi(\lambda \backslash \mathcal{D}) \propto \iint \pi\left(\lambda, \rho, \beta \backslash \tau_{1}, \tau_{2}, \mathcal{D}\right) d \rho d \beta \\
& =\lambda^{d_{3}-1} e^{-\lambda\left[S_{1}\left(\tau_{1}^{*}, \tau_{2}^{*}\right)\right]} \iint \rho^{d_{3}-d_{1}\left(\tau_{1}^{*}, \tau_{2}^{*}\right)-1} e^{\left\{\beta\left(d_{3}^{(2)}-d_{1}^{(2)}\right)-\lambda \rho S_{2}\left(\tau_{1}^{*}, \tau_{2}^{*}, \beta\right)\right\}} d \rho d \beta \\
& =\lambda^{d_{3}-1} \exp \left\{-\lambda\left[S_{1}\left(\tau_{1}^{*}, \tau_{2}^{*}\right)\right]\right\} \frac{\Gamma\left[d_{3}-d_{1}\left(\tau_{1}^{*}, \tau_{2}^{*}\right)\right]}{\lambda^{d_{3}-d_{1}\left(\tau_{1}^{*}, \tau_{2}^{*}\right)}} \int \frac{\exp \left\{\beta\left(d_{3}^{(2)}-d_{1}^{(2)}\right)\right\}}{\left[S_{2}\left(\tau_{1}^{*}, \tau_{2}^{*}, \beta\right)\right]^{d_{3}-d_{1} i \tau_{1}^{*}, \tau_{2}^{*} !}} d \beta
\end{aligned}
$$

assim,

$$
\begin{aligned}
& \pi(\lambda \backslash \mathcal{D}) \propto \\
& \propto \lambda^{d_{1}\left(\tau_{1}^{*}, \tau_{2}^{*}\right)-1} \exp \left\{-\lambda\left[S_{1}\left(\tau_{1}^{*}, \tau_{2}^{*}\right)\right]\right\} \int \exp \left\{\beta\left[d_{3}^{(2)}-d_{1}^{(2)}-d_{3}+d_{1}\left(\tau_{1}^{*}, \tau_{2}^{*}\right)\right]\right\} d \beta \\
& =\lambda^{d_{1}\left(\tau_{1}^{*}, \tau_{2}^{*}\right)-1} \exp \left\{-\lambda\left[S_{1}\left(\tau_{1}^{*}, \tau_{2}^{*}\right)\right]\right\} \int \exp \left\{\beta\left[d_{1}^{(1)}-d_{3}^{(1)}\right]\right\} d \beta
\end{aligned}
$$

isto é,

$$
\pi(\lambda \backslash \mathcal{D}) \propto \lambda^{d_{1}\left(\tau_{1}^{*}, \tau_{2}^{*}\right)-1} \exp \left\{-\lambda\left[S_{1}\left(\tau_{1}^{*}, \tau_{2}^{*}\right)\right]\right\}
$$

portanto,

$$
\lambda \backslash \mathcal{D} \sim \Gamma\left[d_{1}\left(\tau_{1}^{*}, \tau_{2}^{*}\right), S_{1}\left(\tau_{1}^{*}, \tau_{2}^{*}\right)\right]
$$

A média e a moda a posteriori de $\lambda$ são dadas, repectivamente, por,

$$
E(\lambda \backslash \mathcal{D})=\frac{d_{1}\left(\tau_{1}^{*}, \tau_{2}^{*}\right)}{S_{1}\left(\tau_{1}^{*}, \tau_{2}^{*}\right)} \quad M o(\lambda)=\frac{d_{1}\left(\tau_{1}^{*}, \tau_{2}^{*}\right)-1}{S_{1}\left(\tau_{1}^{*}, \tau_{2}^{*}\right)}
$$


A densidade marginal a posteriori de $\rho$ é dada por:

$$
\begin{aligned}
& \pi(\rho \backslash \mathcal{D}) \propto \iint \pi\left(\lambda, \rho, \beta \backslash \tau_{1}, \tau_{2}, \mathcal{D}\right) d \lambda d \beta \\
& =\rho^{d_{3}-d_{1}\left(\tau_{1}^{*}, \tau_{2}^{*}\right)-1} \iint \lambda^{d_{3}-1} e^{\left\{\beta\left(d_{3}^{(2)}-d_{1}^{(2)}\right)-\lambda\left[S_{1}\left(\tau_{1}^{*}, \tau_{2}^{*}\right)+\rho S_{2}\left(\tau_{1}^{*}, \tau_{2}^{*}, \beta\right)\right]\right\}} d \lambda d \beta \\
& =\rho^{d_{3}-d_{1}\left(\tau_{1}^{*}, \tau_{2}^{*}\right)-1} \Gamma\left(d_{3}\right) \int \frac{\exp \left\{\beta\left(d_{3}^{(2)}-d_{1}^{(2)}\right)\right\}}{\left[S_{1}\left(\tau_{1}^{*}, \tau_{2}^{*}\right)+\rho S_{2}\left(\tau_{1}^{*}, \tau_{2}^{*}, \beta\right)\right]^{d_{3}}} d \beta
\end{aligned}
$$

Vamos denotar por $I$ a integral $\int \frac{\exp \left\{\beta\left(d_{3}^{(2)}-d_{1}^{(2)}\right)\right\}}{\left[S_{1}\left(\tau_{1}^{*}, \tau_{2}^{*}\right)+\rho S_{2}\left(\tau_{1}^{*}, \tau_{2}^{*}, \beta\right)\right]^{d_{3}}} d \beta$

Resolvendo a integral $I$ pelo método de aproximação de Laplace, temos

$$
I \approx \rho^{d_{1}^{(2)}-d_{3}^{(2)}}\left[S_{1}\left(\tau_{1}^{*}, \tau_{2}^{*}\right)+\rho D_{1}\right]^{d_{3}^{(2)}-d_{1}^{(2)}-d_{3}}
$$

onde $D_{1}=D_{1}\left(\tau_{1}^{*}\right)=W_{2}^{(1)}\left(\tau_{1}^{*}\right)-\tau_{1}^{*}\left[n_{1}-d_{2}^{(1)}\right]$

então,

$$
\pi(\rho \backslash \mathcal{D}) \propto \rho^{d_{3}^{(1)}-d_{1}^{(1)}-1}\left[S_{1}\left(\tau_{1}^{*}, \tau_{2}^{*}\right)+\rho D_{1}\right]^{d_{3}^{(2)}-d_{1}^{(2)}-d_{3}}
$$

Observar que $\pi(\rho / \mathcal{D})$ não é uma distribuição conhecida e o cálculo da média e da moda a posteriori foi feito usando métodos numericos iterativos.

A densidade marginal a posteriori de $\beta$ é dada por:

$$
\begin{aligned}
& \pi(\beta \backslash \mathcal{D}) \propto \int_{0}^{\infty} \int_{0}^{\infty} \pi\left(\lambda, \rho, \beta \backslash \tau_{1}, \tau_{2}, \mathcal{D}\right) d \rho d \lambda \\
& =e^{\left\{\beta\left(d_{3}^{(2)}-d_{1}^{\prime 2}\right)\right\}} \int_{0}^{\infty} \int_{0}^{\infty} \lambda^{d_{3}-1} \rho^{d_{3}-d_{1}\left(\tau_{1}^{*}, \tau_{2}^{*}\right)-1} e^{\left\{-\lambda\left[S_{1}\left(\tau_{1}, \tau_{2}\right)+\rho S_{2}\left(\tau_{1}, \tau_{2}, \beta\right)\right]\right\}} d \rho d \lambda \\
& =e^{\left\{\beta\left(d_{3}^{\prime 2 !}-d_{1}^{2:}\right)\right\}} \frac{\Gamma\left[d_{3}-d_{1}\left(\tau_{1}^{*}, \tau_{2}^{*}\right)\right]}{\left[S_{2}\left(\tau_{1}^{*}, \tau_{2}^{*}, \beta\right)\right]^{\left.d_{3}-d_{1}^{\prime} \tau_{1}^{*}, \tau_{2}^{*}\right)}} \int_{0}^{\infty} \frac{\lambda^{d_{3}-1}}{\lambda^{d_{3}-d_{1}\left(r_{1}^{*}, \tau_{2}^{*}\right)}} e^{\left\{-\lambda\left[S_{1}\left(\tau_{1}, \tau_{2}\right)\right]\right\}} d \lambda
\end{aligned}
$$




$$
=e^{\left\{\beta\left(d_{3}^{2 j}-d_{1}^{(2)}\right)\right\}} \frac{\Gamma\left[d_{3}-d_{1}\left(\tau_{1}^{*}, \tau_{2}^{*}\right)\right]}{\left[S_{2}\left(\tau_{1}^{*}, \tau_{2}^{*}, \beta\right)\right]^{\left.d_{3}-d_{1} \tau_{1}^{*}, \tau_{2}^{*}\right)}} \frac{\Gamma\left[d_{1}\left(\tau_{1}^{*}, \tau_{2}^{*}\right)\right]}{\left.S_{1}\left(\tau_{1}^{*}, \tau_{2}^{*}\right)\right]^{d_{1}\left(\tau_{1}^{*}, \tau_{2}^{*}\right)}}
$$

isto é,

$\pi(\beta \backslash \mathcal{D}) \propto \frac{e^{\left\{\beta\left(d_{3}^{(2)}-d_{1}^{(2)}\right)\right\}}}{\left[S_{2}\left(\tau_{1}^{*}, \tau_{2}^{*}, \beta\right)\right]^{d_{3}-d_{1}\left(\tau_{1}^{*}, \tau_{2}^{*}\right)}}$

Como no caso anterior, $\pi(\beta \backslash \mathcal{D})$ não é uma distribuição conhecida e o cálculo da média e da moda foi feito numericamente.

Considerar a função de verossimilhança dada em (2.7), assumindo pontos de mudança conhecidos e densidades a priori informativas para os parâmetros, dadas por:

$\lambda \sim \operatorname{Gama}\left[a_{1}, b_{1}\right]$

$\rho \sim \operatorname{Gama}\left[a_{2}, b_{2}\right]$

$\beta \sim \operatorname{Normal}\left[\mu_{0}, \sigma_{0}^{2}\right]$.

onde $a_{1}, b_{1}, a_{2}, b_{2}, \mu_{0}$ e $\sigma_{0}^{2}$ são constantes conhecidas.

Com essas densidades a priori a densidade conjunta a posteriori é dada por,

$$
\begin{aligned}
& \pi\left(\lambda, \rho, \beta \backslash \tau_{1}, \tau_{2}, \mathcal{D}\right) \propto \\
& \propto \lambda^{d_{3}+a_{1}-1} \rho^{d_{3}-d_{1}\left(\tau_{1}^{*}, \tau_{2}^{*}\right)+a_{2}-1} e^{\left\{\beta\left(d_{3}^{(2)}-d_{1}^{(2)}\right)\right\}} \times \\
& \exp \left\{-\frac{1}{2 \sigma_{0}^{2}}\left[\beta-\mu_{0}\right]^{2}\right\} \exp \left\{-\lambda\left[b_{1}+S_{1}\left(\tau_{1}^{*}, \tau_{2}^{*}\right)+\rho S_{2}\left(\tau_{1}^{*}, \tau_{2}^{*}, \beta\right)\right]-\rho b_{2}\right\}
\end{aligned}
$$

A densidade marginal a posteriori de $\lambda$ é dada por,

$\pi(\lambda \mid \mathcal{D}) \propto \iint \pi\left(\lambda, \rho, \beta \backslash \tau_{1}, \tau_{2}, \mathcal{D}\right) d \rho d \beta$ 


$$
\begin{aligned}
& \pi(\lambda \backslash \mathcal{D}) \propto \lambda^{d_{3}+a_{1}-1} \exp \left\{-\lambda\left[b_{1}+S_{1}\left(\tau_{1}^{*}, \tau_{2}^{*}\right)\right\} \Gamma\left(d_{3}-d_{1}\left(\tau_{1}^{*}, \tau_{2}^{*}\right)+a_{2)} \times\right.\right. \\
& \int \frac{e^{\left\{\beta\left(d_{3}^{(2)}-d_{1}^{(2)}\right)\right\}} \cdot}{\left.\left[\lambda S_{2}\left(\tau_{1}^{*}, \tau_{2}^{*}, \beta\right)\right]+b_{2}\right]^{d_{3}-d_{1}\left(\tau_{1}^{*}, \tau_{2}^{*}\right)+r_{2}}} \exp \left\{-\frac{1}{2 \sigma_{0}^{2}}\left[\beta-\mu_{0}\right]^{2}\right\} d \beta
\end{aligned}
$$

Usando o método de aproximação de Laplace, encontramos,

$$
\pi(\lambda \backslash \mathcal{D}) \propto \frac{\lambda^{d_{3}+a_{1}-1}}{\left.\left[\lambda S_{2}\left(\tau_{1}^{*}, \tau_{2}^{*}, \beta\right)\right]+b_{2}\right]^{d_{3}-d_{1}\left(\tau_{1}^{*}, \tau_{2}^{*}\right)+a_{2}}} \exp \left\{-\lambda\left[b_{1}+S_{1}\left(\tau_{1}^{*}, \tau_{2}^{*}\right)\right]\right\}
$$

Observar que $\pi(\lambda \mid \mathcal{D})$ não é uma distribuição conhecida; assim a média a posteriori de $\lambda$ também pode ser aproximada pelo método de Laplace.

A densidade a posteriori marginal de $\rho$ é dada por:

$\pi(\rho \backslash \mathcal{D}) \propto \iint \pi\left(\lambda, \rho, \beta \backslash \tau_{1}, \tau_{2}, \mathcal{D}\right) d \lambda d \beta$

isto é,

$$
\begin{aligned}
& \pi(\rho \backslash \mathcal{D}) \propto \rho^{d_{3}-d_{1}\left(\tau_{1}^{*}, \tau_{2}^{*}\right)+a_{2}-1} \exp \left\{-\rho b_{2}\right\} \times \\
& \int \frac{\Gamma\left[d_{3}+a_{1}\right]}{\left.\left[b_{1}+S_{1}\left(\tau_{1}^{*}, \tau_{2}^{*}\right)+\rho S_{2}\left(\tau_{1}^{*}, \tau_{2}^{*}, \beta\right)\right]+b_{2}\right]^{d_{3}+a_{1}}} \exp \left\{\beta\left(d_{3}^{(2)}-d_{1}^{(2)}\right)-\frac{1}{2 \sigma_{0}^{2}}\left[\beta-\mu_{0}\right]^{2}\right\} d \beta
\end{aligned}
$$

Novamente, aproximando esta integral pelo método de Laplace, encontramos,

$$
\pi(\rho \backslash \mathcal{D}) \propto \frac{\rho^{d_{3}-d_{1}\left(\tau_{1}^{*}, \tau_{2}^{*}\right)+a_{2}-1} \exp \left\{-\rho b_{2}\right\}}{\left.\left[b_{1}+S_{1}\left(\tau_{1}^{*}, \tau_{2}^{*}\right)+\rho S_{2}\left(\tau_{1}^{*}, \tau_{2}^{*}, \beta\right)\right]+b_{2}\right]^{d_{3}+a_{1}}}
$$

A média a posteriori de $\rho$ também foi aproximada pelo método de Laplace.

O uso de densidades a priori informativas apresenta mais dificuldades : O cálculo da densidade marginal a posteriori de $\beta$ pode ser dificil pelo método de Laplace. 


\section{Uso de métodos de simulação MCMC}

Assumindo os pontos de mudança conhecidos, considerar a função de verossimilhança dada em (2.13) e a densidade a priori não informativa dada por (2.40); a densidade conjunta a posteriori é dada por:

$\pi\left(\lambda, \rho, \beta \backslash \tau_{1}, \tau_{2}, \mathcal{D}\right) \propto$

$\propto \lambda^{d_{3}-1} \rho^{d_{3}-d_{1}\left(\tau_{1}^{*}, \tau_{2}^{*}\right)-1} \exp \left\{\beta\left(d_{3}^{(2)}-d_{1}^{(2)}\right)-\lambda\left[S_{1}\left(\tau_{1}^{*}, \tau_{2}^{*}\right)+\rho S_{2}\left(\tau_{1}^{*}, \tau_{2}^{*}, \beta\right)\right]\right\}$

onde $S_{1}\left(\tau_{1}, \tau_{2}\right)$ e $S_{2}\left(\tau_{1}, \tau_{2}, \beta\right)$ são dadas em (2.14).

As densidades condicionais a posteriori para o algoritmo amostrador de Gibbs são dadas por,

$\pi\left(\lambda \backslash \rho, \beta, \tau_{1}, \tau_{2}, \mathcal{D}\right) \propto \lambda^{d_{3}-1} \exp ^{\left\{-\lambda\left[S_{1}\left(\tau_{1}^{*}, \tau_{2}^{*}\right)+\rho S_{2}\left(\tau_{1}^{*}, \tau_{2}^{*}, \beta\right)\right]\right\}}$

isto é, $\quad \lambda \backslash \rho, \beta, \tau_{1}, \tau_{2}, \mathcal{D} \sim \operatorname{Gama}\left[d_{3}, S_{1}\left(\tau_{1}^{*}, \tau_{2}^{*}\right)+\rho S_{2}\left(\tau_{1}^{*}, \tau_{2}^{*}, \beta\right)\right]$

$\pi\left(\rho \backslash \lambda, \beta, \tau_{1}, \tau_{2}, \mathcal{D}\right) \propto \rho^{d_{3}-d_{1}\left(\tau_{1}^{*}, \tau_{2}^{*}\right)-1} \exp ^{\left.\left\{-\rho \lambda S_{2}\left(\tau_{1}^{*}, \tau_{2}^{*}, \beta\right)\right]\right\}}$

isto é, $\rho \backslash \lambda, \beta, \tau_{1}, \tau_{2}, \mathcal{D} \sim \operatorname{Gama}\left[d_{3}-d_{1}\left(\tau_{1}^{*}, \tau_{2}^{*}\right), \lambda S_{2}\left(\tau_{1}^{*}, \tau_{2}^{*}, \beta\right)\right]$

Fazendo a reparametrização $\phi=e^{\beta}$ temos,

$\pi\left(\phi \backslash \rho, \lambda, \tau_{1}, \tau_{2}, \mathcal{D}\right) \propto \phi^{d_{3}^{2)}-d_{1}^{(2)}-1} \exp \left\{-\phi\left[\lambda \rho\left[w_{2}^{(2)}-\tau_{2}^{*}\left(n_{2}-d_{2}^{(2)}\right]\right]\right\}\right.$

isto é, $\phi \backslash \rho, \lambda, \tau_{1}, \tau_{2}, \mathcal{D} \sim \operatorname{Gama}\left[d_{3}^{2)}-d_{1}^{(2)}, \lambda \rho\left[w_{2}^{(2)}-\tau_{2}^{*}\left(n_{2}-d_{:}^{(2)}\right]\right]\right.$ 
Considerar agora as densidades a priori informativas para $\lambda, \rho$ e $\beta$ dadas em (2.46). Com essa escolha de densidade a priori, assumindo independência a priori para os parâmetros, a densidade conjunta a posteriori é dada por,

$$
\begin{aligned}
& \pi\left(\lambda, \rho, \beta \backslash \tau_{1}, \tau_{2}, \mathcal{D}\right) \propto \lambda^{d_{3}+a_{1}-1} \rho^{d_{3}-d_{1}\left(\tau_{1}^{*}, \tau_{2}^{*}\right)+a_{2}-1} \times \\
& \exp \left\{-\frac{1}{2 \sigma_{3}^{2}}\left(\beta-\mu_{0}\right)^{2}\right\} \exp \left\{\beta\left(d_{3}^{(2)}-d_{1}^{(2)}\left(\tau_{2}^{*}\right)\right\} \times\right. \\
& \exp \left\{-\lambda\left[b_{1}+S_{1}\left(\tau_{1}^{*}, \tau_{2}^{*}\right)+\rho S_{2}\left(\tau_{1}^{*}, \tau_{2}^{*}, \beta\right)\right]\right\} \exp \left\{-\rho b_{2}\right\}
\end{aligned}
$$

As densidades a posteriori condicionais para o algoritmo amostrador de Gibbs são das por,

$$
\begin{aligned}
& \pi(\lambda \backslash \rho, \beta, \mathcal{D}) \propto \lambda^{d_{3}+a_{1}-1} \exp \left\{-\lambda\left[b_{1}+S_{1}\left(\tau_{1}^{*}, \tau_{2}^{*}\right)+\rho S_{2}\left(\tau_{1}^{*}, \tau_{2}^{*}, \beta\right)\right]\right\} \\
& \text { isto é, } \lambda \backslash \rho, \beta, \mathcal{D} \sim \operatorname{Gama}\left[d_{3}+a_{1}, b_{1}+S_{1}\left(\tau_{1}^{*}, \tau_{2}^{*}\right)+\rho S_{2}\left(\tau_{1}^{*}, \tau_{2}^{*}, \beta\right)\right] \\
& \pi(\rho \backslash \lambda, \beta, \mathcal{D}) \propto \rho^{d_{3}-d_{1}\left(\tau_{1}^{*}, \tau_{2}^{*}\right)+a_{2}-1} \exp \left\{-\rho\left[b_{2}+S_{2}\left(\tau_{1}^{*}, \tau_{2}^{*}, \beta\right)\right]\right\} \\
& \text { isto é, } \rho \backslash \lambda, \beta, \mathcal{D} \sim \operatorname{Gama}\left[d_{3}-d_{1}\left(\tau_{1}^{*}, \tau_{2}^{*}\right)+a_{2}, b_{2}+S_{2}\left(\tau_{1}^{*}, \tau_{2}^{*}, \beta\right)\right]
\end{aligned}
$$

e

$$
\begin{aligned}
& \pi(\beta \backslash \lambda, \rho, \mathcal{D}) \propto \exp \left\{-\frac{1}{2 \sigma_{0}^{2}}\left(\beta-\mu_{0}\right)^{2}\right\} \exp \left\{\beta\left(d_{3}^{(2)}-d_{1}^{(2)}\left(\tau_{2}^{*}\right)\right\} \times\right. \\
& \exp \left\{-\lambda \rho S_{2}\left(\tau_{1}^{*}, \tau_{2}^{*}, \beta\right)\right\}
\end{aligned}
$$

Observar que $\pi(\beta \backslash \lambda, \rho, \mathcal{D})$ não é uma distribuição conhecida; então é preciso usar o algoritmo Metropolis-Hastings para gerar uma amostra de $\beta$. Para isso, reescrevemos $\pi(\beta \backslash \lambda, \rho, \mathcal{D})=q(\beta) \psi(\beta)$, onde $q(\beta)$ é a densidade Normal, $N\left(\mu_{0}, \sigma_{0}^{2}\right)$ que é usada como núcleo para gerar $\beta$ e $\psi(\beta)$ é dado por,

$$
\psi(\beta)=\exp \left\{\beta\left(d_{3}^{\prime 2)}-d_{1}^{\prime 2}\left(\tau_{2}^{*}\right)\right\} \exp \left\{-\lambda \rho S_{2}\left(\tau_{1}^{*}, \tau_{2}^{*}, \beta\right)\right\}\right.
$$




\section{Capítulo 3}

\section{Modelo de sobrevivência com função de risco variável no tempo}

\subsection{Apresentação do modelo}

A função de risco constante apresentada nos modelos do capítulo anterior, muitas vezes não é a mais adequada. Para esses casos é preciso assumir uma função de risco que varia com o tempo. Neste capítulo apresentamos um modelo assumindo a distribuição Weibull para os tempos de vida, obtendo assim resultados mais realistas.

\subsubsection{Modelo Weibull com um ponto de mudança - Um tratamento}

A função de risco, assumindo uma distribuição Weibull para os tempos de vida é dada por:

$h(t)= \begin{cases}\lambda \gamma t^{\gamma-1} & , t<\tau \\ \lambda \alpha \gamma t^{\alpha \gamma-1} & , t \geq \tau\end{cases}$

onde $\lambda>0$ é o parâmetro de escala, $\gamma>0$ é parâmetro de forma e $\alpha>0$ é o parâmetro que reflete a mudança na função de risco.

A função densidade de probabilidade e a função de sobrevivência associadas à $h(t)$ são, respectivamente

$$
\begin{aligned}
& f(t)= \begin{cases}\lambda \gamma t^{\gamma-1} \exp \left\{-\lambda t^{\gamma}\right\} & , t<\tau \\
\lambda \alpha \gamma t^{\alpha \gamma-1} \exp \left\{-\lambda\left[\tau^{\gamma}+t^{\alpha \gamma}-\tau^{\alpha \gamma}\right]\right\} & , t \geq \tau\end{cases} \\
& S(t)= \begin{cases}\exp \left\{-\lambda t^{\gamma}\right\} & , t<\tau \\
\exp \left\{-\lambda\left[\tau^{\gamma}+t^{\alpha \gamma}-\tau^{\alpha \gamma}\right]\right\} & , t \geq \tau\end{cases} \\
& \lambda>0, \gamma>0 \text { e } \alpha>0 .
\end{aligned}
$$


Para estimar o ponto de mudança graficamente, observamos a relação entre a função de risco e a função de sobrevivência. No caso da distribuição Weibull com um ponto de mudança temos,

$H(t)=\left\{\begin{array}{lcl}\lambda t^{\gamma} & \text { se } & t<\tau \\ \lambda\left[\tau^{\gamma}+t^{\alpha \gamma}-\tau^{\alpha \gamma}\right] & \text { se } & t \geq \tau\end{array}\right.$

Dai,

$\log [H(t)]=\left\{\begin{array}{lr}\log \lambda+\gamma \log t & \text { se } t<\tau \\ \log \left[\lambda t^{\alpha \gamma}+\lambda\left(\tau^{\gamma}-\tau^{\alpha \gamma}\right)\right] & \text { se } t \geq \tau\end{array}\right.$

A partir da linearidade entre $\log H(t)$ e $\log t$ podemos estimar graficamente o ponto de mudança $\tau$. Com dados censurados podemos usar o estimador produto-limite de Kaplan e Meier (1958) para a função de sobrevivência.

\subsection{Inferência clássica}

Para esse modelo, considerando dados com censuras do tipo I progressivas, podemos estimar graficamente o ponto de mudança na função de risco, calcular os estimadores de máxima verossimilhança e intervalos de confiança para os outros parâmetros $(\lambda, \gamma$ e $\alpha)$.

A função de verossimilhança assumindo distribuição Weibull para os tempos de vida é dada por:

$$
\begin{aligned}
& L(\lambda, \gamma, \alpha, \tau)= \\
& \quad \prod_{i=1}^{n}\left\{\left[\lambda \gamma t_{i}^{\gamma-1} \exp \left\{-\lambda t^{\gamma}\right\}\right]^{\epsilon_{i}}\left[\lambda \alpha \gamma t_{i}^{\alpha \gamma-1} \exp \left\{-\lambda\left[\tau^{\gamma}+t_{i}^{\alpha \gamma}-\tau^{\alpha \gamma}\right]\right\}\right]^{1-\epsilon_{i}}\right\}^{\delta_{i}} \\
& \prod_{i=1}^{n}\left\{\left[\exp \left\{-\lambda t_{i}^{\gamma}\right\}\right]^{\epsilon_{i}}\left[\exp \left\{-\lambda\left[\tau^{\gamma}+t_{i}^{\alpha \gamma}-\tau^{\alpha \gamma}\right]\right\}\right]^{1-\epsilon_{i}}\right\}^{1-\delta_{i}}
\end{aligned}
$$

onde $\delta$ e $\epsilon$ são variáveis indicadoras definidas da mesma forma que para os modelos anteriores (ver seção 2.2.1, no capítulo 2).

Definindo $\quad d_{1}(\tau)=\sum_{i=1}^{n} \epsilon_{i} \delta_{i}, d_{2}(\tau)=\sum_{i=1}^{n} \epsilon_{i}$ e $d_{3}=\sum_{i=1}^{n} \delta_{i}$, podemos simplificar (3.4) como segue: 


$$
\begin{aligned}
L(\lambda, \gamma, \alpha, \tau)= & (\lambda \gamma)^{d_{3}} \alpha^{d_{3}-d_{1}(\tau)} \prod_{i=1}^{n}\left[t_{i}^{\gamma-1}\right]^{\epsilon_{i} \delta_{i}} \prod_{i=1}^{n}\left[t_{i}^{\alpha \gamma-1}\right]^{\left(1-\epsilon_{i}\right) \delta_{i}} \\
& \exp \left\{-\lambda\left[\sum_{i=1}^{n} \epsilon_{i} t_{i}^{\gamma}+\sum_{i=1}^{n}\left(1-\epsilon_{i}\right) t_{i}^{\alpha \gamma}+\left[n-d_{2}(\tau)\right]\left[\tau^{\gamma}-\tau^{\alpha \gamma}\right]\right]\right\}
\end{aligned}
$$

Assumindo o ponto de mudança conhecido e denotando $l(\lambda, \gamma, \alpha)=\ln [L(\lambda, \gamma, \alpha)]$ e derivando com relação aos parâmetros $\lambda, \gamma$ e $\alpha$, temos:

$$
\begin{aligned}
\frac{\partial l}{\partial \lambda}= & \frac{d_{3}}{\lambda}-\sum_{i=1}^{n} \epsilon_{i} t_{i}^{\gamma}-\sum_{i=1}^{n}\left(1-\epsilon_{i}\right) t_{i}^{\alpha \gamma}-\left[n-d_{2}\left(\tau^{*}\right)\right]\left[\tau^{* \gamma}-\tau^{* \alpha \gamma}\right] \\
\frac{\partial l}{\partial \gamma}= & \frac{d_{3}}{\gamma}+\sum_{i=1}^{n} \epsilon_{i} \delta_{i} l n\left(t_{i}\right)+\alpha \sum_{i=1}^{n}\left(1-\epsilon_{i}\right) \delta_{i} \ln \left(t_{i}\right)-\lambda \sum_{i=1}^{n} \epsilon_{i} t_{i}^{\gamma} \ln \left(t_{i}\right)- \\
& -\lambda \alpha \sum_{i=1}^{n}\left(1-\epsilon_{i}\right) t_{i}^{\alpha \gamma} \ln \left(t_{i}\right)-\lambda\left[n-d_{2}\left(\tau^{*}\right)\right] \ln \left(\tau^{*}\right)\left[\tau^{* \gamma}-\alpha \tau^{* \alpha \gamma}\right] \\
\frac{\partial l}{\partial \alpha}= & \frac{d_{3}-d_{1}\left(\tau^{*}\right)}{\alpha}+\gamma \sum_{i=1}^{n}\left(1-\epsilon_{i}\right) \delta_{i} \ln \left(t_{i}\right)- \\
& -\lambda \gamma \sum_{i=1}^{n}\left(1-\epsilon_{i}\right) t_{i}^{\alpha \gamma} \ln \left(t_{i}\right)-\lambda \gamma\left[n-d_{2}\left(\tau^{*}\right)\right] \ln \left(\tau^{*}\right) \tau^{* \alpha \gamma}
\end{aligned}
$$

Definimos funções $S_{i}(),. i=1, \ldots, 8 ; r_{j}(),. j=1, \ldots, 4$ como segue:

$$
\begin{array}{lll}
S_{1}(\gamma, \tau)=\sum_{i=1}^{n} \epsilon_{i} t_{i}^{\gamma} & S_{2}(\alpha, \gamma, \tau)=\sum_{i=1}^{n}\left(1-\epsilon_{i}\right) t_{i}^{\alpha \gamma} \\
S_{3}(\tau)=\sum_{i=1}^{n} \epsilon_{i} \delta_{i} l n\left(t_{i}\right) & , & S_{4}(\tau)=\sum_{i=1}^{n}\left(1-\epsilon_{i}\right) \delta_{i} l n\left(t_{i}\right) \\
S_{5}(\gamma, \tau)=\sum_{i=1}^{n} \epsilon_{i} t_{i}^{\gamma} l n\left(t_{i}\right) & , & S_{6}(\alpha, \gamma, \tau)=\sum_{i=1}^{n}\left(1-\epsilon_{i}\right) t_{i}^{\alpha \gamma} \ln \left(t_{i}\right) \\
S_{7}(\gamma, \tau)=\sum_{i=1}^{n} \epsilon_{i} t_{i}^{\gamma}\left[\ln \left(t_{i}\right)\right]^{2} & , & S_{8}(\alpha, \gamma, \tau)=\sum_{i=1}^{n}\left(1-\epsilon_{i}\right) t_{i}^{\alpha \gamma}\left[\ln \left(t_{i}\right)\right]^{2} \\
r_{1}(\alpha, \gamma, \tau)=\tau^{\gamma}-\tau^{\alpha \gamma} & , & r_{2}(\alpha, \gamma, \tau)=\ln (\tau)\left[\tau^{\gamma}-\alpha \tau^{\alpha \gamma}\right] \\
r_{3}(\alpha, \gamma, \tau)=[\ln (\tau)]^{2}\left[\tau^{\gamma}-\alpha^{2} \tau^{\alpha \gamma}\right] & , \quad r_{4}(\alpha, \gamma, \tau)=\tau^{\alpha \gamma} \ln (\tau)[1+\alpha \gamma \ln (\tau)]
\end{array}
$$

Para simplificar a notação consideramos $S_{i}()=.S_{i}, i=1, \ldots, 8$ e $r_{j}()=.r_{j}$, $j=1, \ldots, 4$. 
Fazendo $\frac{\partial l}{\partial \lambda}=0$, temos $\hat{\lambda}=\frac{d_{3}}{S_{1}+S_{2}+\left[n-d_{2}\left(\tau^{*}\right)\right] r_{1}}$

Substituindo (3.10) em (3.7) e (3.8) temos um sistema de duas equações não lineares envolvendo apenas $\gamma$ e $\alpha$, dado por:

$$
\begin{aligned}
& g_{1}(\alpha, \gamma)=\frac{\partial l}{\partial \gamma}=\frac{S_{3}+\alpha S_{4}+d_{3}\left\{\frac{1}{\gamma}-S_{5}-\alpha S_{6}-\left[n-d_{2}(\tau)\right] r_{2}\right\}}{D}=0 \\
& g_{2}(\alpha, \gamma)=\frac{\partial l}{\partial \alpha}=\frac{d_{3}-d_{1}}{\alpha}+\gamma S_{4}-\frac{d_{3} \gamma\left\{S_{6}-\left[n-d_{2}(\tau)\right] \ln (\tau) \tau^{\alpha \gamma}\right\}}{D}=0
\end{aligned}
$$

Os EMV são obtidos resolvendo o sistema de equações não-lineares (3.11). Para isso, usamos o método iterativo de Newton-Raphson [ver por exemplo, Lee, E.T. 1980] : Denotando por $\gamma^{(k)}$ e $\alpha^{(k)}$ os valores dos parâmetros na $k$-ésima iteração, a atualização desses valores é feita como segue:

$$
\left(\begin{array}{l}
\gamma^{(k+1)} \\
\alpha^{(k+1)}
\end{array}\right)=\left(\begin{array}{l}
\gamma^{(k)} \\
\alpha^{(k)}
\end{array}\right)-\left(\begin{array}{ll}
w_{11} & w_{12} \\
w_{21} & w_{22}
\end{array}\right)\left(\begin{array}{l}
g_{1}\left(\alpha^{(k)}, \gamma^{(k)}\right) \\
g_{2}\left(\alpha^{(k)}, \gamma^{(k)}\right)
\end{array}\right)
$$

onde $W=\left\{w_{i j}\right\}$ é a inversa da matriz de derivadas segundas $G=\left\{g_{i j}\right\}$ dada por :

$$
\begin{aligned}
g_{11}=\frac{\partial^{2} l}{\partial \gamma^{2}}= & \frac{\left\{S_{5}+\alpha S_{6}+\left[n-d_{2}(\tau)\right] r_{2}\right\}\left\{S_{5}+\alpha S_{6}+\left[n-d_{2}(\tau)\right] \ln (\tau)\left[\tau^{\gamma}+\alpha \tau^{\alpha \gamma}\right]\right\}}{D^{2}}- \\
& -\frac{d_{3}\left\{S_{7}+\alpha S_{8}+\left[n-d_{2}(\tau)\right] r_{3}\right\}}{D} \\
g_{12}=\frac{\partial^{2} l}{\partial \gamma \partial \alpha}= & \frac{\gamma\left\{S_{5}+\alpha S_{6}+\left[n-d_{2}(\tau)\right] r_{2}\right\}\left\{S_{6}+\left[n-d_{2}(\tau)\right] \ln (\tau) \tau^{\alpha \gamma}\right\}}{D^{2}}- \\
& -\frac{d_{3}\left\{S_{6}+\alpha \gamma S_{8}+\left[n-d_{2}(\tau)\right] r_{4}\right\}}{D} \\
g_{21}=\frac{\partial^{2} l}{\partial \alpha \partial \gamma}= & S_{4}+\frac{d_{3} \gamma\left\{S_{6}-\left[n-d_{2}(\tau)\right] \ln (\tau) \tau^{\alpha \gamma}\right\}\left\{S_{5}+\alpha S_{6}+\left[n-d_{2}(\tau)\right] \ln (\tau)\left[\tau^{\gamma}+\alpha \tau^{\alpha \gamma}\right]\right\}}{D^{2}}- \\
& -\frac{d_{3}\left\{S_{6}-\left[n-d_{2}(\tau)\right] \ln (\tau) \tau^{\alpha \gamma}+\alpha \gamma\left(S_{8}-\left[n-d_{2}(\tau)\right][\ln (\tau)]^{2} \tau^{\alpha \gamma}\right)\right\}}{D}
\end{aligned}
$$




$$
\begin{aligned}
g_{22}=\frac{\partial^{2} l}{\partial \alpha^{2}}= & \frac{d_{1}(\tau)-d_{3}}{\alpha^{2}}+\frac{d_{3} \gamma^{2}\left\{S_{6}-\left[n-d_{2}(\tau)\right] \ln (\tau) \tau^{\alpha \tau}\right\}\left\{S_{6}+\left[n-d_{2}(\tau)\right] \ln (\tau) \tau^{\alpha \tau}\right\}}{D^{2}}- \\
& -\frac{d_{3} \gamma^{2}\left\{S_{8}-\left[n-d_{2}(\tau)\right][\ln (\tau)]^{2} \tau^{\alpha \tau}\right\}}{D}
\end{aligned}
$$

onde $D=S_{1}+S_{2}+\left[n-d_{2}(\tau)\right]\left[\tau^{\gamma}+\tau^{\alpha \gamma}\right]$

No capítulo 5 apresentamos uma aplicação desse método para dados reais.

\subsection{Inferência Bayesiana}

Para a abordagem Bayesiana deste modelo com $\tau$ conhecido, consideramos uma densidade conjunta a priori não informativa de Jeffreys assumindo independência entre os parâmetros $\lambda, \gamma$ e $\alpha$,

$$
\pi_{0}(\lambda, \gamma, \alpha) \propto \frac{1}{\lambda \gamma \alpha}
$$

A densidade conjunta a posteriori é calculada combinando a função de verossimilhança (3.5) e a densidade conjunta a priori (3.17) resultando em

$$
\begin{aligned}
\pi(\lambda, \gamma, \alpha \backslash \tau, \mathcal{D}) & \propto(\lambda \gamma)^{d_{3}-1} \alpha^{d_{3}-d_{1}\left(\tau^{*}\right)} \prod_{i=1}^{n}\left[t_{i}^{\gamma-1}\right]^{\epsilon_{i} \delta_{i}} \prod_{i=1}^{n}\left[t_{i}^{\alpha \gamma-1}\right]^{\left(1-\epsilon_{i}\right) \delta_{i}} \times \\
& \exp \left\{-\lambda\left[\sum_{i=1}^{n} \epsilon_{i} t_{i}^{\gamma}+\sum_{i=1}^{n}\left(1-\epsilon_{i}\right) t_{i}^{\alpha \gamma}+\left[n-d_{2}\left(\tau^{*}\right)\right]\left[\tau^{* \gamma}-\tau^{* \alpha \gamma}\right]\right]\right\}
\end{aligned}
$$

A densidade conjunta a posteriori (3.18) apresenta um grau de complexidade suficiente para justificar a opção pelos métodos de simulação de MCMC para obter estimativas para os parâmetros. As densidades condicionais a posteriori para os algoritmos amostrador de Gibbs e Metropolis-Hastings são dadas por:

$\pi(\lambda \backslash \alpha, \gamma, \tau, \mathcal{D}) \propto$

$\lambda^{d_{3}-1} \exp \left\{-\lambda\left[\sum_{i=1}^{n} \epsilon_{i} t_{i}^{\gamma}+\sum_{i=1}^{n}\left(1-\epsilon_{i}\right) t_{i}^{\alpha \gamma}+\left[n-d_{2}\left(\tau^{*}\right)\right]\left[\tau^{* \gamma}-\tau^{* \alpha \gamma}\right]\right]\right\}$ 


$$
\begin{aligned}
& \pi(\alpha \backslash \lambda, \gamma, \tau, \mathcal{D}) \propto \\
& \alpha^{d_{3}-d_{1}\left(\tau^{*}\right)} \exp \left\{\alpha \gamma\left[\sum_{i=1}^{n}\left(1-\epsilon_{i}\right) \delta_{i} l n\left(t_{i}\right)\right]\right\} \exp \left\{-\lambda\left[\sum_{i=1}^{n}\left(1-\epsilon_{i}\right) t_{i}^{\alpha \gamma}-\left[n-d_{2}\left(\tau^{*}\right)\right] \tau^{* \alpha \gamma}\right]\right\} \\
& \pi(\gamma \backslash \lambda, \alpha, \tau, \mathcal{D}) \propto \gamma^{d_{3}-1} \exp \left\{\gamma\left[\sum_{i=1}^{n} \epsilon_{i} \delta_{i} \ln \left(t_{i}\right)+\alpha \sum_{i=1}^{n}\left(1-\epsilon_{i}\right) \delta_{i} \ln \left(t_{i}\right)\right]\right\} \times \\
& \exp \left\{-\lambda\left[\sum_{i=1}^{n} \epsilon_{i} t_{i}^{\gamma}+\sum_{i=1}^{n}\left(1-\epsilon_{i}\right) t_{i}^{\alpha \gamma}+\left[n-d_{2}\left(\tau^{*}\right)\right]\left[\tau^{* \gamma}-\tau^{* \alpha \gamma}\right]\right]\right\}
\end{aligned}
$$

Usando as expressões $S_{i}$ e $r_{j}$ dadas em (3.9), temos:

$$
\pi(\lambda \backslash \alpha, \gamma, \tau, \mathcal{D}) \propto \lambda^{d_{3}-1} \exp \left\{-\lambda\left[S_{1}+S_{2}+\left[n-d_{2}\left(\tau^{*}\right)\right] r_{1}\right]\right\}
$$

isto é , $\lambda \backslash \alpha, \gamma, \tau, \mathcal{D} \sim \operatorname{Gama}\left[d_{3}, S_{1}+S_{2}+\left[n-d_{2}\left(\tau^{*}\right)\right] r_{1}\right]$,

$$
\pi(\alpha \backslash \lambda, \gamma, \tau, \mathcal{D}) \propto \alpha^{d_{3}-d_{1}\left(\tau^{*}\right)-1} \exp \left\{\alpha \gamma S_{4}-\lambda\left[S_{2}-\left[n-d_{2}\left(\tau^{*}\right)\right] \tau^{* \alpha \gamma}\right]\right\}
$$

e

$$
\pi(\gamma \backslash \lambda, \alpha, \tau, \mathcal{D}) \propto \gamma^{d_{3}-1} \exp \left\{\gamma\left[S_{3}+\alpha S_{4}-\lambda\left[S_{1}+S_{2}+\left[n-d_{2}\left(\tau^{*}\right)\right] r_{1}\right]\right\}\right.
$$

Com estas densidades condicionais, utilizamos o algoritmo amostrador de Gibbs para gerar a amostra de $\lambda$ e para gerar amostras de $\alpha$ e $\gamma$, pelo algoritmo Metropolis-Hastings, adotamos como núcleos $q_{1}(\alpha)$ e $q_{2}(\gamma)$, densidades Gama. 
Considerar agora o ponto de mudança desconhecido assumindo valores discretos $\tau_{i}=t_{i}$, com densidade a priori $\pi_{0}\left(\tau_{i}=t_{i}\right)=\frac{1}{n}, i=1,2, \ldots, n$. Assim a densidade conjunta a priori para $\lambda, \gamma, \alpha$ e $\tau_{i}$ é dada por,

$\pi\left(\lambda, \gamma, \alpha, \tau_{i}\right)=\pi_{0}\left(\lambda, \gamma, \alpha \backslash \tau_{i}\right) \pi_{0}\left(\tau_{i}=t_{i}\right)=\frac{1}{\lambda \gamma \alpha n}$

Com a densidade conjunta a posteriori dada em (3.18), as densidades condicionais a posteriori para os algoritmos amostrador de Gibbs e Metropolis-Hastings são dadas por:

$\pi\left(\lambda \backslash \alpha, \gamma_{2} \tau, \mathcal{D}\right) \propto \lambda^{d_{3}-1} \exp \left\{-\lambda\left[S_{1}+S_{2}+\left[n-d_{2}(\tau)\right] r_{1}\right]\right\}$

isto é , $\lambda \backslash \alpha, \gamma, \tau, \mathcal{D} \sim \operatorname{Gama}\left[d_{3}, S_{1}+S_{2}+\left[n-d_{2}(\tau)\right] r_{1}\right]$

$\pi(\alpha \backslash \lambda, \gamma, \tau, \mathcal{D}) \propto \alpha^{d_{3}-d_{1}(\tau)-1} \exp \left\{\alpha \gamma S_{4}-\lambda\left[S_{2}-\left[n-d_{2}(\tau)\right] \tau^{\alpha \gamma}\right]\right\}$

$\pi(\gamma \backslash \lambda, \alpha, \tau, \mathcal{D}) \propto \gamma^{d_{3}-1} \exp \left\{\gamma\left[S_{3}+\alpha S_{4}-\lambda\left[S_{1}+S_{2}+\left[n-d_{2}(\tau)\right] r_{1}\right]\right\}\right.$

$\pi(\tau \backslash \gamma, \lambda, \alpha) \propto \alpha^{-d_{1}(\tau)} \exp \left\{\gamma\left[S_{3}+\alpha S_{4}\right]-\lambda\left[S_{1}+S_{2}+\left[n-d_{2}(\tau)\right] r_{1}(\tau)\right]\right\}$

Para $\lambda$ podemos usar o algoritmo amostrador de Gibbs enquanto que para $\alpha, \gamma$ e $\tau$ usamos o algoritmo Metropolis-Hastings. Geramos amostras de $\alpha$ e $\gamma$ com os mesmos núcleos $q(\alpha)$ e $q(\gamma)$ adotados anteriormente e para gerar uma amostra de $\tau$ usamos um núcleo uniforme discreto $U(n)$. 


\section{Capítulo 4}

\section{Processo de Poisson com pontos de mudança}

\subsection{Apresentação dos modelos}

Considerar um processo de Poisson homogêneo com parâmetro $\lambda$. O processo é observado no intervalo de tempo $[0, T]$ durante o qual ocorrem $n$ eventos nos instantes $t_{1}, \ldots, t_{n}$. Denotamos por $N(T)=n$ o número de eventos que ocorrem até $T$. A determinação dos pontos de mudança na taxa do processo é feita graficamente observando a relação entre $N\left(t_{i}\right) \mathrm{e}$ $t_{i}$. Esta relação pode ser obtida a partir dos estimadores de máxima verossimilhança dos parâmetros do modelo como vamos apresentar na próxima seção deste capítulo.

\subsubsection{Processo de Poisson com um ponto de mudança}

Assumindo que há apenas um ponto de mudança, a taxa de ocorrência no instante $t_{i}$ é dada por

$$
\lambda\left(t_{i}\right)=\left\{\begin{array}{llr}
\lambda_{1} & \text { se } 0 \leq t_{i} \leq \tau \\
\lambda_{2} & \text { se } & t_{i}>\tau
\end{array}\right.
$$

Seja $X$ os intervalos de tempo entre as ocorrências. A função densidade para $X$, é dada por

$$
f\left(x_{i}\right)= \begin{cases}\lambda_{1} e^{-\lambda_{1} x_{i}} & \text { se } \sum_{j=1}^{i} x_{j} \leq \tau \\ \lambda_{2} e^{-\lambda_{2} x_{i}} & \text { se } \sum_{j=1}^{i} x_{j} \geq \tau\end{cases}
$$

sendo $x_{i}=t_{i}-t_{i-1}, i=1, \ldots, n$

$\sum_{i=1}^{n} x_{i}=\left(t_{1}-t_{0}\right)+\left(t_{2}-t_{1}\right)+\ldots+\left(t_{n}-t_{n-1}\right)=t_{n}-t_{0}=T$

As expressões (4.1) e (4.2) podem ser extendidas facilmente para problemas com mais de um ponto de mudança. 


\subsubsection{Processo de Poisson com dois pontos de mudança}

Considerar um processo de Poisson com dois pontos de mudança com taxa de ocorrência no instante $t_{i}$ dada por:

$$
\lambda\left(t_{i}\right)= \begin{cases}\lambda_{1} & \text { se } 0<t_{i} \leq \tau_{1} \\ \lambda_{2} & \text { se } \tau_{1}<t_{i} \leq \tau_{2} \\ \lambda_{3} & \text { se } \tau_{2}<t_{i} \leq T\end{cases}
$$

A função densidade dos intervalos de tempo entre as ocorrências, $X_{i}$ é dada por:

$$
f\left(x_{i}\right)= \begin{cases}\lambda_{1} e^{-\lambda_{1} x_{i}} & \text { se } i=0, \ldots, \tau_{1} \\ \lambda_{2} e^{-\lambda_{2} x_{i}} & \text { se } i=\tau_{1}+1, \ldots, \tau_{2} \\ \lambda_{3} e^{-\lambda_{3} x_{i}} & \text { se } i=\tau_{2}+1, \ldots, T\end{cases}
$$

\subsection{Inferência Clássica}

Uma forma de verificar se existe um ou mais pontos de mudança na taxa de ocorrência do processo é observar o gráfico de $N\left(t_{i}\right) \times t_{i}$. Por exemplo, considerar um processo com um único ponto de mudança, com taxa de ocorrência no instante $t_{i}$ dada em (4.1). Considerando a função densidade para $X_{i}(4.2)$ e definindo uma variável indicadora $\epsilon$ tal que

$$
\epsilon\left(x_{i}\right)=\left\{\begin{array}{cc}
1 & \text { se } \sum_{j=1}^{i} x_{j} \leq \tau \\
0 & \text { se } \sum_{j=1}^{i} x_{j} \geq \tau
\end{array}\right.
$$

A função de verossimilhança para $\lambda_{1}, \lambda_{2}$ e $\tau$ é dada por

$$
L\left(\lambda_{1}, \lambda_{2}, \tau\right)=\prod_{i=1}^{n}\left(\lambda_{1} e^{-\lambda_{1} x_{i}}\right)^{\epsilon_{i}}\left(\lambda_{2} e^{-\lambda_{2} x_{i}}\right)^{1-\epsilon_{i}}
$$

Simplificando,

$$
L\left(\lambda_{1}, \lambda_{2}, \tau\right)=\lambda_{1}^{N(\tau)} e^{-\lambda_{1} \tau} \lambda_{2}^{n-N(\tau)} e^{-\lambda_{2}(T-\tau)}
$$

onde $N(\tau)$ é o número de eventos que ocorrem até $\tau$. 
Os estimadores de máxima verossimilhança para $\lambda_{1}$ e $\lambda_{2}$ são dados por:

$$
\widehat{\lambda}_{1}=\frac{N(\tau)}{\tau} \quad \text { e } \quad \widehat{\lambda}_{2}=\frac{n-N(\tau)}{T-\tau}
$$

Considerando que $\tau$ pode assumir qualquer um dos valores $t_{1}, \ldots, t_{n}$, as expressões dadas em (4.6) implicam na relação abaixo

$$
N\left(t_{i}\right)=\left\{\begin{array}{lr}
\widehat{\lambda}_{1} t_{i} & \text { se } t_{i} \leq \tau \\
\left(n-\widehat{\lambda}_{2} T\right)+\widehat{\lambda}_{2} t_{i} & \text { se } t_{i}>\tau
\end{array}\right.
$$

Essa relação entre $N\left(t_{i}\right)$ e $t_{i}$, permite estimar graficamente o número de pontos de mudança e a localização desses pontos.

A estimativa de máxima verossimilhança para o parâmetro $\tau$ só pode ser obtida calculando $\widehat{\lambda}_{1}$ e $\widehat{\lambda}_{2}$ para todos $\tau_{i}=t_{i}$ e avaliando a função somente nesses pontos. Uma forma de contornar esse problema é usar inferência Bayesiana.

\subsection{Inferência Bayesiana}

\subsubsection{Análise para o processo com um ponto de mudança}

Se o processo de Poisson tem um único ponto de mudança, considerando a abordagem Bayesiana do modelo, podemos estimar as taxas $\lambda_{1}$ e $\lambda_{2}$ e também a localização do ponto $\tau$ calculando a moda a posteriori de cada um desses parâmetros.

Assumindo independência entre os parâmetros e considerando densidades a priori não informativas dadas por:

$\pi_{0}\left(\lambda_{1}\right) \propto \frac{1}{\lambda_{1}}, \quad \pi_{0}\left(\lambda_{2}\right) \propto \frac{1}{\lambda_{2}} \quad$ e $\pi_{0}(\tau) \propto$ constante

A densidade conjunta a posteriori é dada por:

$$
\pi\left(\lambda_{1}, \lambda_{2}, \tau \mid \mathcal{D}\right) \propto \lambda_{1}^{N(\tau)-1} e^{-\lambda_{1} \tau} \lambda_{2}^{n-N(\tau)-1} e^{-\lambda_{2}(T-\tau)}
$$


A densidade marginal a posteriori de $\tau$ é dada por:

$$
\pi(\tau \mid \mathcal{D}) \propto \pi_{0}(\tau) \frac{\Gamma[N(\tau)] \Gamma[n=N(\tau)]}{\tau^{N(\tau)}(T-\tau)^{n-N(\tau)}}
$$

Um estimador por ponto para $\tau$ é a moda de $\pi(\tau \mid \mathcal{D})$, assumindo $\tau_{i}=t_{i}$ e denotando essa moda por $\tilde{\tau}$, as densidades condicionais a posteriori são:

$$
\begin{aligned}
& \lambda_{1} \widetilde{\tau}, \mathcal{D} \sim \Gamma[N(\widetilde{\tau}), \widetilde{\tau}] \\
& \lambda_{2} \widetilde{\tau}, \mathcal{D} \sim \Gamma[n-N(\widetilde{\tau}),(T-\widetilde{\tau})]
\end{aligned}
$$

A desvantagem de se estimar o ponto de mudança pela moda a posteriori de $\tau$ é que assim como a estimativa de máxima verossimilhança, esta também fica restrita aos valores observados $t_{i}$.

Considerando o ponto de mudança desconhecido e assumindo as mesmas densidades a priori não informativas, podemos estimar os parâmetros $\lambda_{1}, \lambda_{2}$ e o ponto de mudança $\tau$ por simulação de Monte Carlo em cadeias de Markov utilizando o algoritmo amostrador de Gibbs para gerar amostras de $\lambda_{1}$ e $\lambda_{2}$ e o algoritmo Metropolis-Hastings para gerar uma amostra de $\tau$ a partir das densidades condicionais a posteriori:

$$
\begin{aligned}
& \lambda_{1} \mid \lambda_{2}, \tau, \mathcal{D} \sim \Gamma\left[r_{1}(\tau), S_{1}(\tau)\right] \\
& \lambda_{2} \mid \lambda_{1}, \tau, \mathcal{D} \sim \Gamma\left[r_{2}(\tau), S_{2}(\tau)\right] \\
& \pi\left(\tau \mid \lambda_{1}, \lambda_{2}, \mathcal{D}\right) \propto \lambda_{1}^{N(\tau)} \lambda_{2}^{-N(\tau)} \exp \left\{-\tau\left(\lambda_{1}-\lambda_{2}\right)\right\}
\end{aligned}
$$

\subsubsection{Análise para o processo com dois pontos de mudança}

Se considerarmos o modelo com dois pontos de mudança (4.4), a função de verossimilhança é dada por,

$$
L\left(\lambda_{1}, \lambda_{2}, \lambda_{3}, \tau_{1}, \tau_{2}\right)=\prod_{j=1}^{3} \prod_{i=1}^{n}\left(\lambda_{j} e^{-\lambda, x_{i}}\right)^{\epsilon_{j}\left(x_{i}\right)}
$$


onde $\epsilon_{j}\left(x_{i}\right)=\left\{\begin{array}{ll}1 & \text { se } \tau_{j-1}<\sum_{j=1}^{i} x_{j} \leq \tau_{j} \\ 0 & \text { caso contrário }\end{array} \quad j=1,2,3 ; \tau_{0}=0, \tau_{3}=T\right.$

Simplificando (4.16), temos $L\left(\lambda_{1}, \lambda_{2}, \lambda_{3}, \tau_{1}, \tau_{2}\right)=\prod_{j=1}^{3} \lambda_{j}^{N\left(\tau_{j}\right)} e^{-\lambda_{j} T_{j}}$

onde $N\left(\tau_{j}\right)=\sum_{i=1}^{n} \epsilon_{j}\left(x_{i}\right)$ e $T_{j}=\sum_{i=1}^{n} x_{i} \epsilon_{j}\left(x_{i}\right)$

Assumindo independência dos parâmetros e densidades a priori não informativas dadas por:

$\pi_{0}\left(\lambda_{j}\right) \propto \frac{1}{\lambda_{j}} \quad j=1,2,3 ; \pi_{0}\left(\tau_{1}\right) \propto$ constante $\quad$ e $\quad \pi_{0}\left(\tau_{2}\right) \propto$ constante

A densidade conjunta a posteriori para os parâmetros é dada por:

$$
\pi\left(\lambda_{1}, \lambda_{2}, \lambda_{3}, \tau_{1}, \tau_{2} \mid \mathcal{D}\right) \propto \prod_{j=1}^{3} \lambda_{j}^{N\left(\tau_{j}\right)-1} e^{-\lambda_{j} T_{j}}
$$

Podemos estimar os parâmetros $\lambda_{1}, \lambda_{2}, \lambda_{3}$ e os pontos de mudança $\tau_{1}$ e $\tau_{2}$ por simulação de Monte Carlo em cadeias de Markov utilizando o algoritmo amostrador de Gibbs para gerar amostras de $\lambda_{1}, \lambda_{2}$ e $\lambda_{3}$ e o algoritmo Metropolis-Hastings para gerar amostras de $\tau_{1}$ e $\tau_{2}$ a partir das densidades condicionais a posteriori:

$$
\begin{aligned}
& \pi\left(\lambda_{j} \mid \lambda_{(j)}, \tau_{1}, \tau_{2}, \mathcal{D}\right) \propto \lambda_{j}^{N\left(\tau_{j}\right)} e^{-\lambda_{j} T_{j}} \\
& \pi\left(\tau_{1} \mid \lambda_{1}, \lambda_{2}, \lambda_{3}, \tau_{2}, \mathcal{D}\right) \propto \lambda_{1}^{N\left(\tau_{1}\right)} e^{-\tau_{1}\left(\lambda_{1}-\lambda_{2}\right)} \\
& \pi\left(\tau_{2} \mid \lambda_{1}, \lambda_{2}, \lambda_{3}, \tau_{1}, \mathcal{D}\right) \propto \lambda_{2}^{N\left(\tau_{2}\right)} e^{-\tau_{2}\left(\lambda_{2}-\lambda_{3}\right)}
\end{aligned}
$$

onde $\underset{(j)}{\lambda}$ é o vetor de $\lambda_{i}, i=1,2,3$ não incluindo $\lambda_{j}$.

O uso das técnicas de simulação de MCMC permite a inferência para um modelo com mais pontos de mudança, o que não seria possível com as técnicas clássicas de inferência. 


\subsubsection{Análise de um modelo hierárquico para o processo com um ponto de mudança}

Uma forma alternativa para resolver o problema de um processo de Poisson com um ponto de mudança é considerar o modelo hierárquico proposto por Carlin, Gelfand e Smith (1992), no qual a variável $Y$ é o número de ocorrências em intervalos regulares de tempo, ordenados cronológicamente. Seja a função densidade para $Y$ dada por:

$f\left(y_{i}\right)= \begin{cases}\frac{\left(\theta_{1} t_{i}\right)^{y_{i}} \exp \left\{-\theta_{1} t_{i}\right\}}{y_{i} !} & i=1, \ldots, K \\ \frac{\left(\theta_{2} t_{i}\right)^{y_{i}} \exp \left\{-\theta_{2} t_{i}\right\}}{y_{i} !} & i=K+1, \ldots, n\end{cases}$

onde $K$ representa o intervalo de tempo no qual ocorre a mudança na taxa de ocorrência.

A função de verossimilhança para $\theta_{1}, \theta_{2}$ e $K$ pode ser escrita como a seguir:

$$
\begin{aligned}
& L\left(\theta_{1}, \theta_{2}, K\right)=\frac{\prod_{i=1}^{n} t_{i} v_{i}}{\prod_{i=1}^{n} y_{i} !} \theta_{1}^{K} \theta_{i=1}^{K} y_{i} \theta_{2}^{i=K+1} y_{i}^{n} \exp \left\{-\theta_{1} \sum_{i=1}^{K} t_{i}-\theta_{2} \sum_{i=K+1}^{n} t_{i}\right\} \\
& =\frac{\prod_{i=1}^{n} t_{i} v_{j}}{\prod_{i=1}^{n} y_{i} !}\left(\frac{\theta_{1}}{\theta_{2}}\right)^{\sum_{i=1}^{K} y_{i}} \theta_{2}^{\sum_{i=K+1}^{n} y_{i}} \exp \left\{\left(\theta_{2}-\theta_{1}\right) \sum_{i=1}^{K} t_{i}-\theta_{2} \sum_{i=1}^{n} t_{i}\right\}
\end{aligned}
$$

Assumindo independência a priori para os parâmetros, no segundo estágio, temos as seguintes densidades a priori,

$\theta_{1} \sim \operatorname{Gama}\left(a_{1}, b_{1}\right), \quad \theta_{2} \sim \operatorname{Gama}\left(a_{2}, b_{2}\right)$ e $K \sim$ Uniforme discreta $(n)$

No terceiro estágio do modelo tomamos $b_{1} \sim \operatorname{Gamainversa}\left(c_{1}, d_{1}\right)$ independente de $b_{2} \sim$ Gama inversa $\left(c_{2}, d_{2}\right)$ e assumimos que $a_{1_{2}} a_{2}, c_{1}, c_{2}, d_{1}$ e $d_{2}$ são conhecidos.

Combinando essas densidades a priori e a função de verossimilhança (4.24), temos a densidade conjunta a posteriori dada por: 


$$
\begin{aligned}
\pi\left(\theta_{1}, \theta_{2}, K, b_{1}, b_{2} \mid \mathcal{D}\right) \propto \theta_{1}^{\sum_{i=1}^{K} y_{i}+a_{1}-1} \theta_{2}^{\sum_{i=K+1}^{n} y_{i}+a_{2}-1} b_{1}^{a_{1}-\left(c_{1}-1\right)} b_{2}^{a_{2}-\left(c_{2}-1\right)} \\
\\
\exp \left\{\frac{b_{1}}{d_{1}}+\frac{b_{2}}{d_{2}}-\theta_{1}\left(\sum_{i=1}^{K} t_{i}+b_{1}^{-1}\right)-\theta_{2}\left(\sum_{i=K+1}^{n} t_{i}+b_{2}^{-1}\right)\right\}
\end{aligned}
$$

De (4.26) podemos facilmente escrever as densidades condicionais a posteriori para o uso dos algoritmos de simulação MCMC:

$$
\begin{aligned}
& \pi\left(\theta_{1} \mid \theta_{2}, K, b_{1}, b_{2}, \mathcal{D}\right) \propto \theta_{1}^{\sum_{i=1}^{K} y_{i}+a_{1}-1} \exp \left\{-\theta_{1}\left(\sum_{i=1}^{K} t_{i}+b_{1}^{-1}\right)\right\} \\
& \pi\left(\theta_{2} \mid \theta_{1}, K, b_{1}, b_{2}, \mathcal{D}\right) \propto \theta_{2}^{\sum_{i=K+1}^{n} y_{i}+a_{2}-1} \exp \left\{-\theta_{2}\left(\sum_{i=K+1}^{n} t_{i}+b_{2}^{-1}\right)\right\} \\
& \pi\left(b_{1} \mid \theta_{1}, \theta_{2}, K, b_{2}, \mathcal{D}\right) \propto b_{1}^{a_{1}-\left(c_{1}-1\right)} \exp \left\{-b_{1}^{-1}\left(\theta_{1}+d_{1}^{-1}\right)\right\} \\
& \pi\left(b_{2} \mid \theta_{1}, \theta_{2}, K, b_{1}, \mathcal{D}\right) \propto b_{2}^{a_{2}-\left(c_{2}-1\right)} \exp \left\{-b_{2}^{-1}\left(\theta_{2}+d_{2}^{-1}\right)\right\} \\
& \pi\left(K \mid \theta_{1}, \theta_{2}, b_{1}, b_{2}, \mathcal{D}\right) \propto\left(\frac{\theta_{1}}{\theta_{2}}\right)^{\sum_{i=1}^{K} y_{i}} \exp \left\{\left(\theta_{2}-\theta_{1}\right) \sum_{i=1}^{K} t_{i}-\theta_{2} \sum_{i=1}^{n} t_{i}\right\}
\end{aligned}
$$

Observamos que para gerar uma amostra de $K$ temos que usar o algoritmo MetropolisHastings. Para isso, escolhemos para núcleo uma uniforme discreta $U(n)$.

No capítulo 5 comparamos as estimativas obtidas com os dois modelos Bayesianos para o processo de Poisson com um ponto de mudança, para os dados de número anual de acidentes em minas de carvão, introduzidos por Jarrett (1979).

Para esse mesmo conjunto de dados apresentamos os resultados de um critério Bayesiano (ver apêndice) para a comparação dos modelos com um e dois pontos de mudança. 


\section{Capítulo 5}

\section{Aplicação dos modelos propostos}

Neste capítulo apresentamos aplicações dos modelos propostos. Calculamos estimativas de máxima verossimilhança e também inferência com os métodos Bayesianos.

O cálculo das estimativas clássicas apresenta algumas dificuldades e em alguns casos foi preciso utilizar métodos iterativos obtendo resultados aproximados.

Com a abordagem Bayesiana encontramos o mesmo tipo de dificuldade quando usamos o método de aproximação de integrais de Laplace, já que este método também requer o cálculo do máximo da função de verossimilhança ou da densidade a posteriori envolvida.

Os métodos de simulação de Monte Carlo em cadeias de Markov (ver apêndice) permitem o cálculo das médias e modas a posteriori sem dificuldades. Neste trabalho, utilizamos para todos os modelos o seguinte esquema de seleção das amostras : Foram gerados 10000 valores, sendo 5 cadeias de 2000 valores, cada uma gerada com um valor inicial diferente. Desprezamos os primeiros 500 valores de cada cadeia e selecionamos uma amostra final de tamanho 500, tomando de 15 em 15 dos 1500 valores restantes, ou seja, a amostra final é formada pelos valores das iterações: $515^{\mathrm{a}}, 530^{\mathrm{a}}, 545^{\mathrm{a}}, \ldots, 2000^{\mathrm{a}}$ de cada uma das 5 cadeias.

\subsection{Aplicação para o modelo exponencial com um tratamento}

Uma aplicação do modelo exponencial com um tratamento foi feita para um conjunto de dados de tempos até remissão em leucemia não linfoblástica aguda, assumindo censuras tipo I progressivas [Matthews,D.E. e Farewell, V.T., 1982] apresentados abaixo:

Tabela I - Tempos até remissão (em dias)

Observacões não censuradas (51 pacientes)

\begin{tabular}{lllllllllll}
\hline 24 & 82 & 111 & 152 & 197 & 249 & 270 & 304 & 487 & 534 & 1160 \\
46 & 89 & 117 & 166 & 209 & 254 & 273 & 332 & 510 & 608 & \\
57 & 90 & 128 & 171 & 223 & 258 & 284 & 341 & 516 & 642 & \\
64 & 90 & 143 & 186 & 230 & 264 & 294 & 393 & 518 & 697 & \\
65 & 90 & 148 & 191 & 247 & 269 & 304 & 395 & 518 & 955 & \\
\hline
\end{tabular}


(continuação da Tabela I)

Observações censuradas ( 33 pacientes)

\begin{tabular}{lllllllllll}
\hline 68 & 182 & 182 & 182 & 182 & 182 & 182 & 182 & 182 & 1310 & 1908 \\
119 & 182 & 182 & 182 & 182 & 182 & 182 & 182 & 182 & 1538 & 1966 \\
182 & 182 & 182 & 182 & 182 & 182 & 182 & 182 & 583 & 1634 & 2057 \\
\hline
\end{tabular}

\section{Estimação gráfica de $\tau$}

Como foi visto no capitulo 2, a estimativa gráfica de $\tau$ pode ser obtida através do gráfico de $\widehat{H}(t) \times t$. Na figura 1, temos esse gráfico para o modelo exponencial com um tratamento.

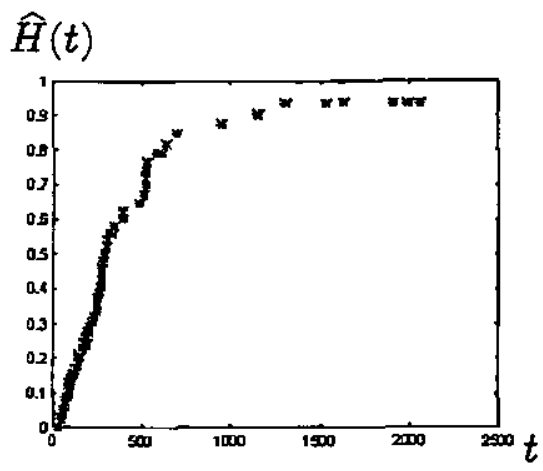

Figura 1 - Gráfico de $\hat{H}_{T}(t) \times t$ para os dados da tabela I

Observando o gráfico, podemos estimar o ponto de mudança em $t \approx 697$. Como essa estimativa gráfica está próxima do valor encontrado com o estimador de máxima verossimilhança, podemos considerar o ponto de mudança conhecido e igual a 697, numa primeira etapa da análise dos dados.

Os estimadores de máxima verossimilhança e os intervalos aproximados com $95 \%$ de confiança para os parâmetros, considerando $\tau=697$, são apresentados na tabela II.

Tabela II - Resultados clássicos

\begin{tabular}{|l|l|l|}
\hline & EMV & I.C. $(95 \%)$ \\
\hline$\lambda$ & 0.002042 & $(0.001862 ; 0.002222)$ \\
\hline$\rho$ & 0.210461 & $(0.1727 ; 0.2482)$ \\
\hline
\end{tabular}

Nas tabelas III e IV temos estimadores Bayesianos dos parâmetros $\lambda$ e $\rho$ considerando densidades a priori não informativas (2.22) e informativas (2.28) usando o método de aproximação de Laplace e o método de simulação MCMC (ver apêndice), respectivamente. Na tabela IV temos também os intervalos de credibilidade para cada parâmetro e a redução potencial da escala estimada, $\widehat{R}$ [ver Gelman,A.E. e Rubin,D., 
1992]. Observamos que o número de iterações considerado é suficiente para obter convergência, já que $\sqrt{\widehat{R}}<1.1$ para os dois parâmetros.

Tabela III - Resultados com aproximação de Laplace ( $\operatorname{com} \tau=697)$ :

Priori não informativa Priori informativa

\begin{tabular}{|l|l|l|l|l|}
\hline & Média & Moda & Média & Moda \\
\hline$\lambda$ & 0.002042 & 0.00199 & 0.002079 & 0.002079 \\
\hline$\rho$ & 0.2137 & 0.1374 & 0.2112 & 0.2112 \\
\hline
\end{tabular}

Tabela IV - Resultados com simulação de Monte Carlo ( $\operatorname{com} \tau=697)$ :

Densidade a priori não informativa

\begin{tabular}{|l|l|l|l|l|}
\hline & Média & Moda & $\widehat{R}$ & Int. de credibilidade \\
\hline$\lambda$ & 0.0020477 & 0.0020104 & 1.0028 & $(0.001494 ; 0.002605)$ \\
\hline$\rho$ & 0.214436 & 0.12513 & 1.0011 & $(0.046755 ; 0.524703)$ \\
\hline
\end{tabular}

Densidade a priori informativa

\begin{tabular}{|l|l|l|l|l|}
\hline & Média & Moda & $\widehat{R}$ & Int. de credibilidade \\
\hline$\lambda$ & 0.002033 & 0.001816 & 0.9993 & $(0.001519 ; 0.002632)$ \\
\hline$\rho$ & 0.209965 & 0.2404 & 0.9972 & $(0.122393 ; 0.298940)$ \\
\hline
\end{tabular}

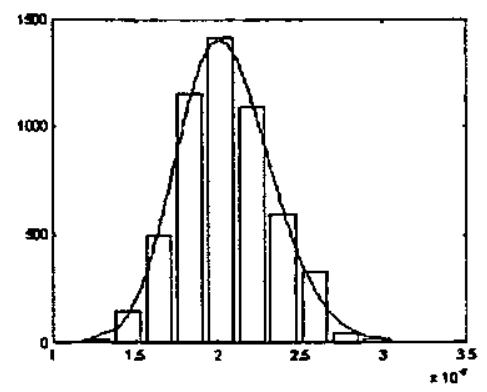

a) $\lambda$

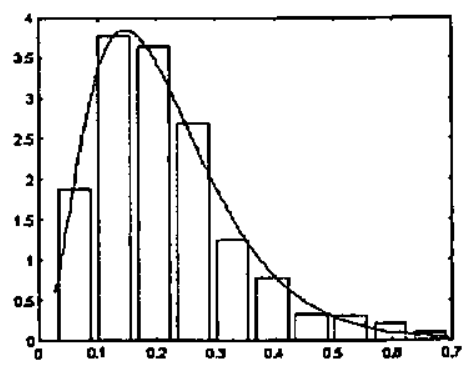

b) $\rho$

Figura. 2 - Densidades marginais a posteriori (Com densidade a priori não informativa)

Podemos observar na figura 2a) que a densidade marginal a posteriori de $\lambda$, calculada com a densidade a priori não informativa pode ser considerada simétrica enquanto que em $2 \mathrm{~b}$ ) a densidade marginal a posteriori de $\rho$ é claramente assimétrica, o que mostra que os resultados da tabela IV estão coerentes: A média e a moda de $\lambda$ estão próximas e a média e a moda de $\rho$ não coincidem. Esse fato justifica o cálculo dos intervalos de credibilidade usando as densidades marginais a posteriori ao invés de calcular intervalos de confiança baseados na normalidade assintótica dos estimadores de máxima verossimilhança, pois esses resultados podem não ter boa precisão. 


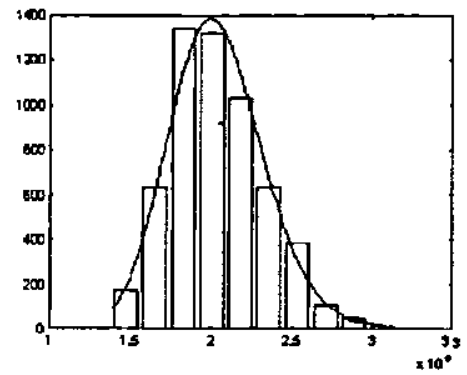

a) $\lambda$

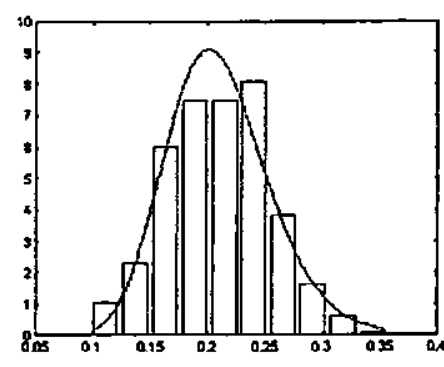

b) $\rho$

Figura 3 - Densidades marginais a posteriori (Com densidade a priori informativa)

$\mathrm{Na}$ figura 3a) observamos menor simetria na densidade marginal a posteriori de $\lambda$ com relação à densidade dada na figura 2a), mostrando o efeito da densidade a priori informativa. Como visto na tabela IV, com densidade a priori informativa, as médias e modas não coincidem tanto para $\lambda$ como para $\rho$.

Considerando agora o ponto de mudança desconhecido e a densidade a priori não informativa (2.22), com o algoritmo amostrador de Gibbs geramos amostras para $\lambda$ e $\rho$ e com o algoritmo Metropolis-Hastings geramos uma amostra para $\tau$.

$\mathrm{Na}$ tabela $\mathrm{V}$ temos estimadores Bayesianos e intervalos de credibilidade para os parâmetros $\tau, \lambda$ e $\rho$. Observamos que o número de iterações considerado é suficiente para obter convergência, já que $\sqrt{\widehat{R}}<1.1$ para os três parâmetros.

Tabela V - Resultados com simulação de Monte Carlo (com $\tau$ desconhecido)

Priori não informativa

\begin{tabular}{|l|l|l|l|l|}
\hline & Média & Moda & $\widehat{\mathrm{R}}$ & Int. de credibilidade \\
\hline$\tau$ & 704.3491 & 730.0518 & 1.0084 & $(539.7803 ; 789.9440)$ \\
\hline$\lambda$ & 0.0020 & 0.0020 & 1.0016 & $(0.0015 ; 0.4550)$ \\
\hline$\rho$ & 0.1602 & 0.1161 & 1.0000 & $(0.0209 ; 0.4550)$ \\
\hline
\end{tabular}

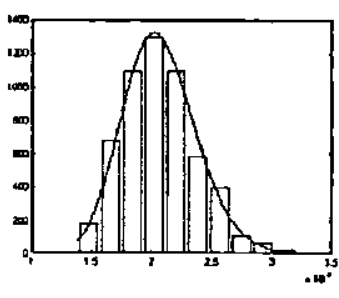

a) $\lambda$

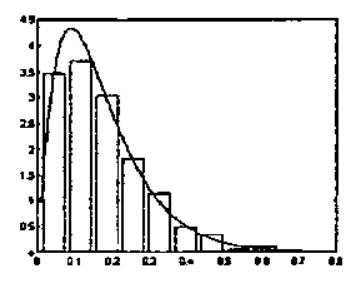

b) $\rho$

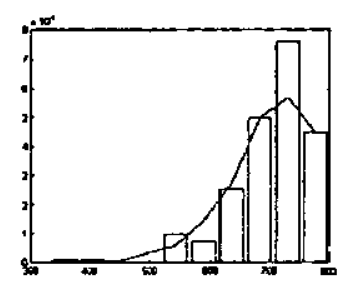

c) $\tau$

Figura 4 - Densidades marginais a posteriori (Com densidade a priori não informativa) 
Observamos na figura 4a) uma simetria razoável na densidade de $\lambda$ enquanto que em 4b) e 4c) podemos notar que as densidades de $\rho$ e $\tau$ são claramente assimétricas, confirmando os resultados apresentados na tabela V.

\section{Interpretação dos resultados:}

Considerando a estimativa gráfica para o ponto de mudança (697 dias) ou a estimativa obtida por simulação (704 dias), os métodos Bayesianos forneceram estimativas para os parâmetros, $\lambda$ e $\rho$, próximas às obtidas maximizando a função de verossimilhança.

Podemos estimar a função de risco para pacientes com leucemia não linfoblástica aguda considerando as modas a posteriori calculadas com densidades a priori informativas (resultados apresentados na tabela IV), como a seguir.

Função de risco estimada para pacientes com leucemia não linfoblástica aguda:

$\widehat{\lambda}(t) \approx \begin{cases}0.002033 & \text { se } t<697 \text { dias } \\ 0.0004269 & \text { se } t \geq 697 \text { dias }\end{cases}$

Este resultado mostra que para pacientes que têm a doença há menos de dois anos (aproximadamente), a chance de ter remissão é muito maior do que para pacientes que já estão com a doença há mais tempo. 


\subsection{Aplicação para o modelo exponencial com dois tratamentos:}

Para uma aplicação do modelo exponencial com dois tratamentos consideramos dados gerados com distribuição exponencial sem ocorrência de censuras (tempos de sobrevida para dois grupos de pacientes, onde cada grupo recebe um tipo de tratamento médico) apresentados na tabela abaixo.

Tabela VI - Tempos de sobrevida em dias

$$
\text { Tratamento 1: } \mathrm{n}_{1}=40
$$

\begin{tabular}{llllllllll}
\hline 0.2 & 1.4 & 2.3 & 5.0 & 7.8 & 11.2 & 15.0 & 48.9 & 121.1 & 230.6 \\
0.5 & 1.5 & 3.2 & 5.4 & 8.8 & 11.5 & 39.5 & 63.0 & 121.2 & 253.5 \\
0.7 & 1.6 & 4.0 & 7.0 & 9.4 & 13.8 & 44.4 & 71.5 & 198.5 & 303.0 \\
0.8 & 2.1 & 4.6 & 7.3 & 10.0 & 14.0 & 46.8 & 115.4 & 218.6 & 365.9 \\
\hline
\end{tabular}

Tratamento 2: $\mathrm{n}_{2}=50$

\begin{tabular}{rrrrrrrrrr}
\hline 0.15 & 1.26 & 1.81 & 6.00 & 7.20 & 10.5 & 287 & 1288 & 1909 & 3551 \\
0.16 & 1.48 & 2.03 & 6.20 & 7.70 & 14.6 & 450 & 1361 & 1951 & 3946 \\
0.82 & 1.55 & 2.90 & 6.40 & 8.50 & 16.9 & 860 & 1433 & 2531 & 5721 \\
0.92 & 1.71 & 4.10 & 6.80 & 9.10 & 21.2 & 1041 & 1560 & 3004 & 6050 \\
1.24 & 1.72 & 5.80 & 7.00 & 9.80 & 25.0 & 1246 & 1670 & 3045 & 6083 \\
\hline
\end{tabular}

A estimação gráfica dos pontos de mudança no risco dos dois grupos de pacientes também pode ser feita como visto no capítulo 2. Para esses dados, os pontos de mudança são evidentes: Com o tratamento 1 , a mudança ocorre após 15 dias e para o tratamento 2 a mudança ocorre após 25 dias.

Os estimadores de máxima verossimilhança e os intervalos aproximados com $95 \%$ de confiança estão na tabela VII.

Tabela VII - Resultados clássicos

\begin{tabular}{|l|l|l|}
\hline & EMV & I.C. (95\%) \\
\hline$\lambda$ & 0.04978 & $(0.03639 ; 0.06317)$ \\
\hline$\rho$ & 0.1594 & $(0.0702 ; 0.2486)$ \\
\hline$\beta$ & -2.9079 & $(-3.5579 ;-2.2579)$ \\
\hline
\end{tabular}


$\mathrm{Na}$ tabela VIII temos os estimadores Bayesianos para $\lambda, \rho$ e $\beta$ obtidos pelo método de aproximação de Laplace com a densidade a priori não informativa dada em (2.40) e também com a densidade a priori informativa dada em (2.46).

Tabela VIII - Resultados com aproximação de Laplace ( $\operatorname{com} \tau_{1}=15$ e $\left.\tau_{2}=25\right)$

Priori não informativa Priori informativa

\begin{tabular}{|l|l|l|l|l|}
\hline & Média & Moda & Média & Moda \\
\hline$\lambda$ & 0.04978 & 0.04884 & 0.05746 & 0.05746 \\
\hline$\rho$ & 0.1624 & 0.1466 & 0.1574 & 0.1574 \\
\hline$\beta$ & -2.9002 & -2.9078 & & \\
\hline
\end{tabular}

Na tabela IX temos os estimadores Bayesianos obtidos por simulação com as densidades a priori citadas acima. Também nesta tabela encontramos os intervalos de credibilidade e a a redução potencial da escala estimada, $\widehat{R}$, para os três parâmetros. Temos que $\sqrt{\widehat{R}}<1.1$ para todos os parâmetros, ou seja, temos convergência do algoritmos amostrador de Gibbs eMetropolis-Hastings.

Tabela IX - Resultados com simulação de Monte Carlo (com $\tau_{1}=15$ e $\left.\tau_{2}=25\right)$

Densidade a priori não informativa

\begin{tabular}{|l|l|l|l|l|}
\hline & Média & Moda & $\widehat{R}$ & Int. de credibilidade \\
\hline$\lambda$ & 0.04989 & 0.04489 & 1.0026 & $(0.038433 ; 0.062625)$ \\
\hline$\rho$ & 0.1632 & 0.16088 & 1.0063 & $(0.081329 ; 0.25651)$ \\
\hline$\beta$ & -2.9113 & -3.0311 & 0.9995 & $(-3.46119 ;-2.24522)$ \\
\hline
\end{tabular}

Densidade a priori informativa

\begin{tabular}{|l|l|l|l|l|}
\hline & Média & Moda & $\widehat{R}$ & Int. de credibilidade \\
\hline$\lambda$ & 0.0501 & 0.0482 & 1.0044 & $(0.042267 ; 0.059199)$ \\
\hline$\rho$ & 0.1579 & 0.1630 & 1.0004 & $(0.111294 ; 0.211644)$ \\
\hline$\beta$ & -2.8974 & -2.9293 & 1.0025 & $(-3.10109 ;-2.69234)$ \\
\hline
\end{tabular}

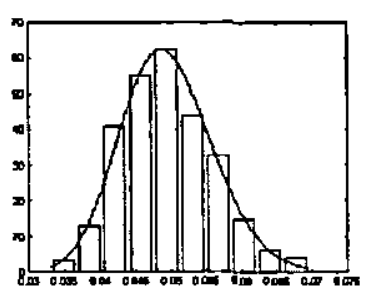

a) $\lambda$

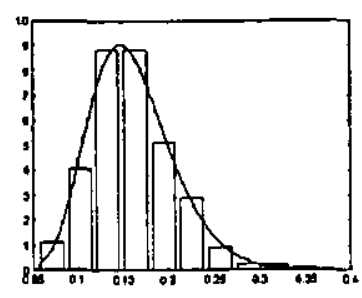

b) $\rho$

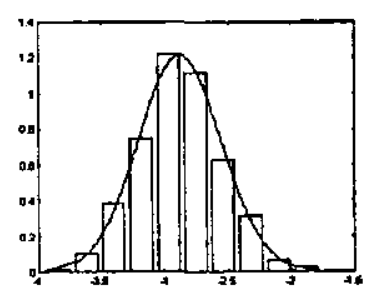

c) $\beta$

Figura 5 - Densidades marginais a posteriori (Com densidade a priori não informativa) 
Observamos na figura 5a) simetria razoável na densidade marginal a posteriori de $\lambda$ e no caso de $\rho$ e $\beta$, em 5 b) e 5c) podemos ver que as densidades apresentam menos simetria, condizendo com os resultados apresentados na tabela IX.

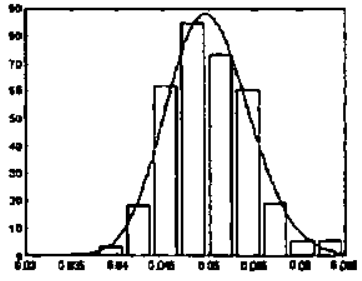

a) $\lambda$

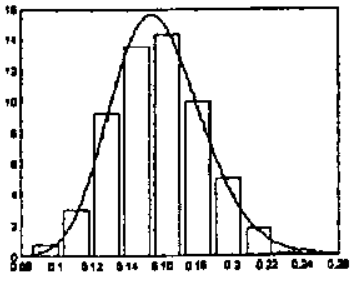

b) $\rho$

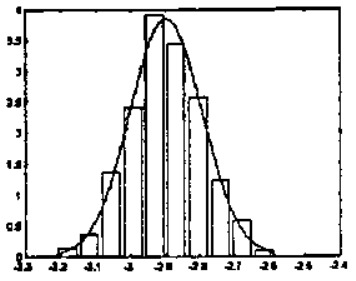

c) $\beta$

Figura 6 - Densidades marginais a posteriori (com densidade a priori informativa)

$\mathrm{Na}$ figura 6 (a,b e c) temos simetria nas densidades marginais a posteriori de $\lambda, \rho \mathrm{e}$ $\beta$. Isto se confirma com os resultados da tabela IX.

\section{Interpretação dos resultados:}

Os resultados Bayesianos se aproximam dos obtidos por máxima verossimilhança. Podemos estimar a função de risco para cada tratamento considerando as modas a posteriori com densidade a priori informativa (tabela IX), como segue:

$$
\begin{aligned}
& \lambda_{1}(t)= \begin{cases}0.0482 & \text { se } t<15 \text { dias } \\
0.0078566 & \text { se } t \geq 15 \text { dias }\end{cases} \\
& \lambda_{2}(t)= \begin{cases}0.0482 & \text { se } t<25 \text { dias } \\
0.0004198 & \text { se } t \geq 25 \text { dias }\end{cases}
\end{aligned}
$$

Esses resultados mostram que para os pacientes que recebem o tratamento tipo 2,o risco de morrer é significativamente menor do que para os pacientes que recebem o tratamento tipo 1. Podemos considerar, portanto, que o tratamento tipo 2 é o mais eficiênte. 


\subsection{Aplicação com o modelo Weibull com um tratamento}

Uma aplicação do modelo Weibull foi feita com os mesmos dados de tempos de remissão (Matthews e Farewell, 1982) dados na tabela I.

Considerando a estimativa gráfica obtida anteriormente, $\tau=697$, os estimadores de máxima verossimilhança para os parâmetros são dados por: $\widehat{\gamma}=1.191587, \widehat{\alpha}=0.8048197, \widehat{\gamma}_{2}=0.959013336 \mathrm{e} \widehat{\lambda}=0.000655485$.

Na figura 7 (a,b,c e d) observamos os valores de $\gamma, \alpha, \gamma_{2}=\alpha \gamma$ e $\lambda$ obtidos de forma iterativa pelo método de Newton-Raphson com 1000 iterações. Notamos que a convergência foi obtida após 300 iterações.

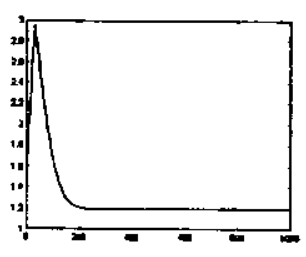

a) $\gamma$

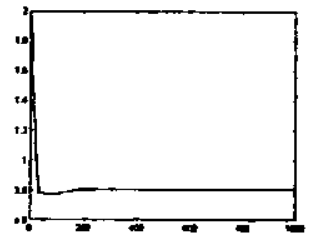

b) $\alpha$

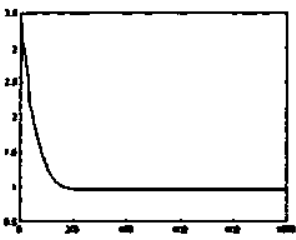

c) $\gamma_{2}=\alpha \gamma$

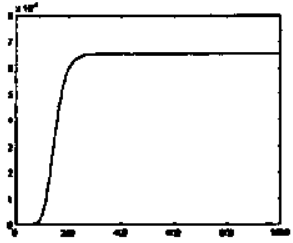

d) $\lambda$

Figura 7 - Convergência do método de Newton-Raphson para $\gamma, \alpha, \gamma_{2}$ e $\lambda$, respectivamente.

Considerando a densidade a priori não informativa dada em (3.17), obtivemos amostras com os algoritmos amostrador de Gibbs para $\lambda$ e Metropolis-Hastings para $\alpha$. A porcentagem de aceitação (ver apêndice), assegura a boa escolha dos núcleos usados para gerar $\alpha$ e $\gamma$ com o algoritmo Metropolis-Hastings. Obtivemos $\alpha$ com aceitação de $50.61 \%$ e $\gamma$ com aceitação de $54.18 \%$. Os resultados estão na tabela $X$.

Tabela X - Resultados com simulação de Monte Carlo (com $\tau=697)$.

\begin{tabular}{|l|l|l|l|l|}
\hline & Média & Moda & $\widehat{R}$ & Int. de credibilidade \\
\hline$\lambda$ & 0.0006127 & 0.0006005 & 0.9988 & $(0.00040129 ; 0.00087233)$ \\
\hline$\gamma$ & 1.2027 & 1.2000 & 1.0024 & $(1.15841 ; 1.247108)$ \\
\hline$\alpha$ & 0.8215 & 0.8179 & 1.0082 & $(0.77188 ; 0.866351)$ \\
\hline
\end{tabular}

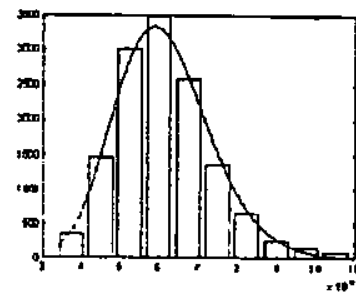

a) $\lambda$

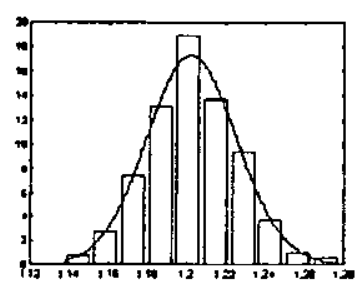

b) $\gamma$

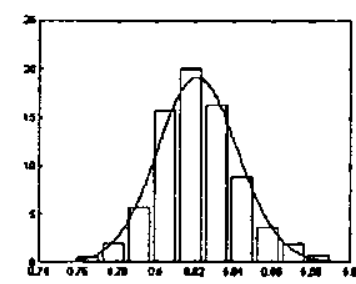

c) $\alpha$

Figura 8 - Densidades marginais a posteriori (Com densidade a priori não informativa). 
Na figura 8 (a,b e c) podemos observar simetria nas densidades marginais a posteriori para $\lambda, \gamma$ e $\alpha$. Esse resultado está confirmado pelos resultados apresentados na tabela X.

Considerando $\tau$ deconhecido e novamente a densidade a priori não informativa dada em (3.17) , geramos amostras com os algoritmos amostrador de Gibbs para $\lambda$ e MetropolisHastings para $\alpha, \gamma$ e $\tau$. Na tabela XI, apresentamos as estimativas para os parâmetros $\lambda, \gamma, \alpha$ e $\tau$

Tabela XI - Resultados com simulação de Monte Carlo ( $\tau$ desconhecido)

\begin{tabular}{|l|l|l|l|l|}
\hline & Média & Moda & $\widehat{R}$ & Int. de credibilidade \\
\hline$\lambda$ & 0.00027 & 0.00024 & 1.0179 & $(0.00017 ; 0.00039)$ \\
\hline$\gamma$ & 1.2296 & 1.2287 & 1.0052 & $(1.1995 ; 1.2534)$ \\
\hline$\alpha$ & 0.8469 & 0.8460 & 1.0192 & $(0.8066 ; 0.8923)$ \\
\hline$\tau$ & 723.627 & 780.1813 & 1.0097 & $(537.2944 ; 797.7528)$ \\
\hline
\end{tabular}

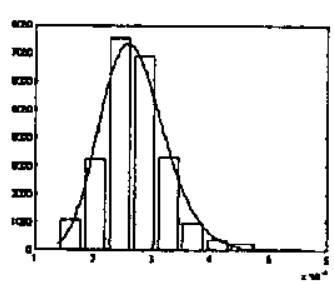

a) $\lambda$

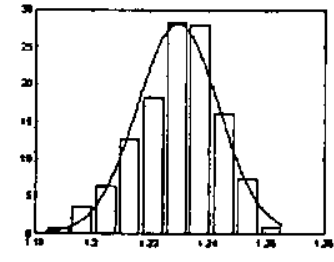

b) $\gamma$

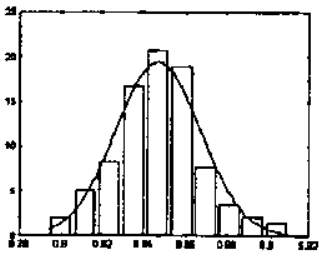

c) $\alpha$

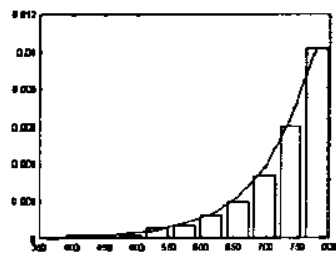

d) $\tau$

Figura 9 - Densidades marginais a posteriori.

A figura 9 (a,b,c) mostra mais simetria nas densidades de $\lambda, \gamma$ e $\alpha$ que na densidade de $\tau$ (figura 9d). Podemos observar isso nos resultados da tabela XI.

\section{Interpretação dos resultados:}

Com os resultados da tabela $\mathrm{XI}$, podemos estimar a função de risco para os pacientes com leucemia não linfoblástica aguda assumindo a distribuição de Weibull para os tempos de remissão (tabela I), como segue:

$h(t)= \begin{cases}0.0002949 t^{0.2287} & \text { se } t<780 \text { dias } \\ 0.0002494 t^{0.03948} & \text { se } t \geq 780 \text { dias }\end{cases}$

Para facilitar a interpretação deste resultado apresentamos um gráfico da função de risco na figura 10. Podemos ver que a função é crescente para qualquer $t$ mas ela cresce mais rapidamente antes do ponto de mudança, mostrando que a chance de haver remissão em pacientes que tem a doença há menos de 780 dias é maior. 


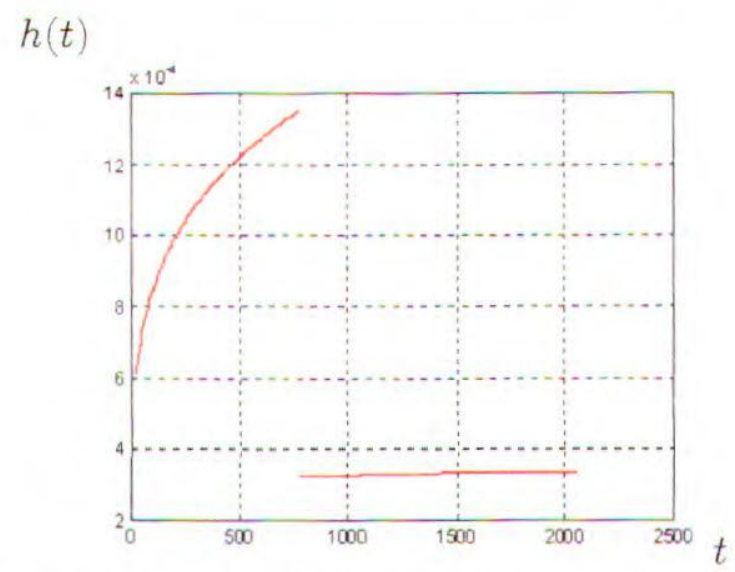

Figura 10 - Gráfico da função de risco Weibull com ponto de mudança

\subsection{Seleção do modelo adequado para os dados da tabela I}

Para a seleção do melhor modelo para os dados da tabela I, consideramos densidades preditivas aproximadas pelos seus estimadores de Monte Carlo baseados nas amostras geradas pelos algoritmos amostrador de Gibbs e Metropolis-Hastings (ver apêndice)

Tabela XII
\begin{tabular}{|l|l|}
\hline$c_{1}$ & $6.3338 \times 10^{-253}$ \\
\hline$c_{2}$ & $2.7229 \times 10^{-255}$ \\
\hline$c_{1}$ & $2.3261 \times 10^{2}$ \\
\hline$c_{2}$ & \\
\hline
\end{tabular}

Na tabela $\mathrm{X}$ temos os valores de $c_{l}, l=1$ para o modelo Weibull e $l=2$ para o modelo exponencial, e a razão $\frac{c_{1}}{c_{2}}$.

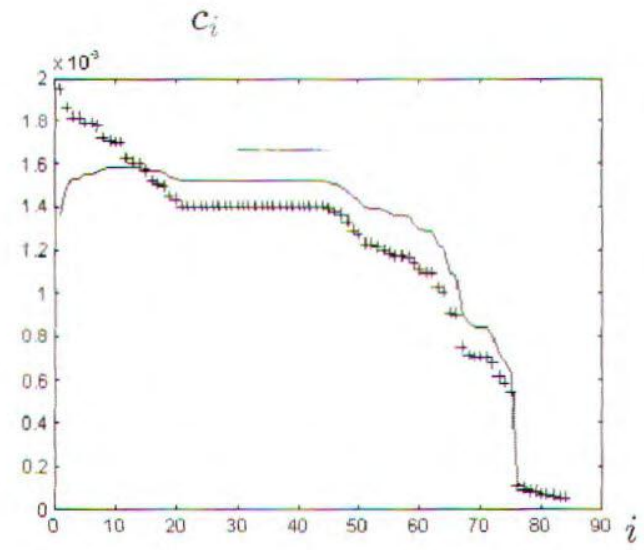

Figura 11 - Gráfico de $c_{l i}$ versus $i, l=1,2 . \quad l=1$ (Weibull ----) e $l=2$ (exponencial ++++ )

Na figura 11 temos o gráfico das preditivas ordenadas estimadas $c_{i}$ versus $t_{i}$ (dados da Tabela I). Observamos um melhor ajuste para o modelo Weibull com um ponto de mudança. 


\subsection{Aplicação para o processo de Poisson com pontos de mudança}

Considerar o conjunto de dados de Maguire, Pearson e Wynn (1952) que representam os intervalos de tempo (em dias) entre explosões em minas de carvão, envolvendo mais de dez mortes, entre 6 de dezembro de 1875 e 29 de maio de 1951 na Inglaterra (tabela XIII), modelado como um processo de Poisson.

Tabela XIII - Intervalos de tempo entre explosões (ordem em linha)

\begin{tabular}{rrrrrrrrrrrrrr}
\hline 378 & 36 & 15 & 31 & 215 & 11 & 137 & 4 & 15 & 72 & 96 & 124 & 50 & 120 \\
203 & 176 & 55 & 93 & 59 & 315 & 59 & 61 & 1 & 13 & 189 & 345 & 20 & 81 \\
286 & 114 & 108 & 188 & 233 & 28 & 22 & 61 & 78 & 99 & 326 & 275 & 54 & 217 \\
113 & 32 & 23 & 151 & 361 & 312 & 354 & 58 & 275 & 78 & 17 & 1205 & 644 & 467 \\
871 & 48 & 123 & 457 & 498 & 49 & 131 & 182 & 255 & 195 & 224 & 566 & 390 & 72 \\
228 & 271 & 208 & 517 & 1613 & 54 & 326 & 1312 & 348 & 745 & 217 & 120 & 275 & 20 \\
66 & 291 & 4 & 369 & 338 & 336 & 19 & 329 & 330 & 312 & 171 & 145 & 75 & 364 \\
37 & 19 & 156 & 47 & 129 & 1630 & 29 & 217 & 7 & 18 & 1357 & & & \\
\hline
\end{tabular}

Iniciamos a análise com o gráfico de $N\left(t_{i}\right) \times t_{i}, i=1, \ldots, 109$ para identificar o número de pontos de mudança, apresentado na figura 12 .

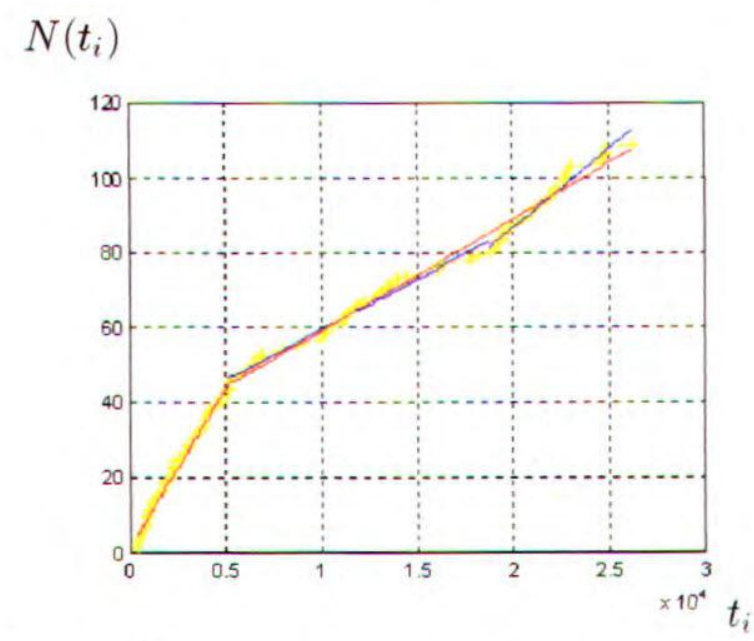

Figura 12: Gráfico de $N\left(t_{i}\right) \times t_{i}$ e ajuste por mínimos quadrados da equação (4.7).

A observação do gráfico da figura 12 sugere a existência de dois pontos de mudança localizados aproximadamente em $t_{45}=5231$ e $t_{81}=19053$. Uma simplificação seria considerar somente o primeiro ponto de mudança. Aqui ajustamos os dois modelos e selecionamos o mais adequado para os dados da tabela XIII, pelo critério das densidades preditivas ordenadas (ver apêndice). 


\subsubsection{Modelo com um ponto de mudança conhecido}

Nesse modelo o ponto de mudança foi estimado pela moda de $\pi(\tau \mid \mathcal{D})$ dada em (4.10), $\tilde{\tau}=5376$ como podemos observar na figura 13. Com esse valor para $\tau$ temos $\lambda_{1}=0.008352$ e $\lambda_{2}=0.002942$ estimados das densidades dadas em (4.11) e (4.12).

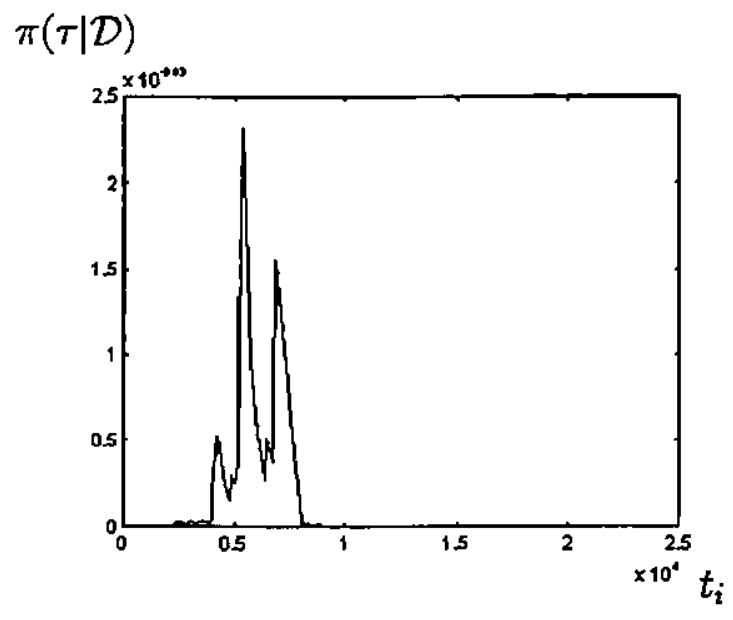

Figura 13 - Densidade marginal a posteriori de $\tau$

\subsubsection{Aplicação para o modelo com um ponto de mudança desconhecido $\left(M_{1}\right)$}

Neste caso, os parâmetros $\lambda_{1}, \lambda_{2}$ e $\tau$ foram estimados usando simulação de Monte Carlo em cadeias de Markov. Para a geração da amostra de $\tau$ foi utilizado o algoritmo Metropolis-Hastings com um núcleo Uniforme[3000,7000]. Um resumo dos resultados dessa simulação é dado na tabela XIV.

Tabela XIV - Resultados da simulação MCMC para o modelo $M_{1}$

\begin{tabular}{|l|l|l|l|l|l|}
\hline var. & média & $\mathrm{dp}$ & moda & $\mathrm{R}$ & \% aceitação \\
\hline$\tau$ & 5674 & 762 & 5311 & 0.9989 & 36.87 \\
\hline$\lambda_{1}$ & 0.0081 & 0.0013 & 0.0076 & 1.0023 & - \\
\hline$\lambda_{2}$ & 0.0030 & 0.0004 & 0.0031 & 1.0025 & - \\
\hline
\end{tabular}

Observamos na tabela XIV que a média de $\lambda_{1}$ é maior que a média $\lambda_{2}$ o que é coerente com $\circ$ gráfico da figura 15. A localização do ponto de mudança, moda de $\tau$, também pode ser vișto na figura 16 . 


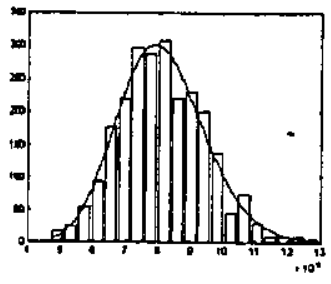

a) $\lambda_{1}$

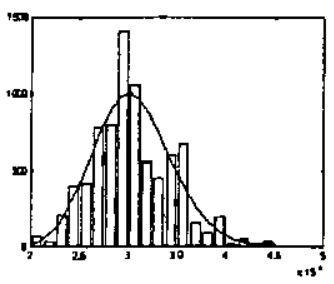

b) $\lambda_{2}$

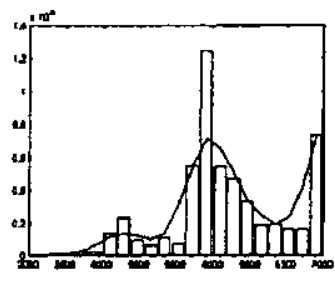

c) $\tau$

Figura 14 - Densidades marginais a posteriori estimadas de $\lambda_{1}, \lambda_{2}$ e $\tau$.

$\mathrm{Na}$ figura 14a) e 14b) observamos simetria nas densidades de $\lambda_{1}$ e $\lambda_{2}$ e na figura 14c) vemos que a densidade de $\tau$ é totalmente assimétrica, inclusive com mais de uma moda.

\section{Interpretação dos resultados:}

Os resultados da tabela XIV mostram que a taxa de ocorrência de explosões cai de 0.0076 ao dia (o que equivale 2.774 ao ano) para 0.0030 ao dia (o que equivale a 1.095 ao ano) após 5674 dias (o que equivale a 15 anos e 6 meses ou seja, a mudança ocorreu no décimo sexto ano observado). Esse modelo permite estimar o ponto de mudança com precisão de dias enquanto que o modelo hierárquico proposto por proposto por Carlin, Gelfand e Smith (1992) (ver seção 4.3.3), só permite determinar em qual ano ocorreu a mudança.

Para comparar esses resultados temos que observar que usamos dados que vão de 1875 à 1951 e portanto o décimo sexto ano é 1890 .

No artigo de Carlin, Gelfand e Smith (1992) as estimativas foram obtidas com dados desde 1851 até 1962. Para esses dados temos as seguintes estimativas: $K=40$ portanto a mudança ocorreu no ano de $1890, \lambda_{1}=3.06$ e $\lambda_{2}=0.89$.

\subsubsection{Aplicação para o modelo com dois pontos de mudança desconhecidos $\left(M_{2}\right)$ :}

Assumindo agora o modelo com dois pontos de mudança, geramos amostras para os 5 parâmetros do modelo usando os métodos de simulação de Monte Carlo em cadeias de Markov. Na tabela $\mathrm{XV}$, temos as médias e modas a posteriori para esses parâmetros e também o valor de $\widehat{R}$. 
Tabela XV - Resultados da simulação MCMC para o modelo $M_{2}$

\begin{tabular}{|l|l|l|l|l|}
\hline & Média & d.p. & Moda & $\widehat{R}$ \\
\hline$\tau_{1}$ & 5030 & 18.3 & 5044 & 1.0072 \\
\hline$\tau_{2}$ & 18681 & 99.0 & 18796 & 1.0084 \\
\hline$\lambda_{1}$ & 0.0081 & 0.0014 & 0.0084 & 1.0015 \\
\hline$\lambda_{2}$ & 0.0024 & 0.0004 & 0.0022 & 1.0050 \\
\hline$\lambda_{3}$ & 0.0039 & 0.0007 & 0.0037 & 0.9980 \\
\hline
\end{tabular}

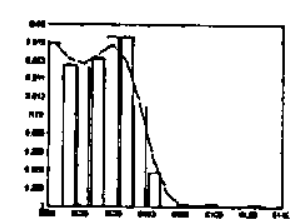

$\tau_{1}$

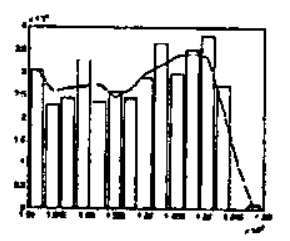

$\tau_{2}$

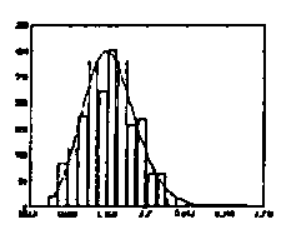

$\lambda_{1}$

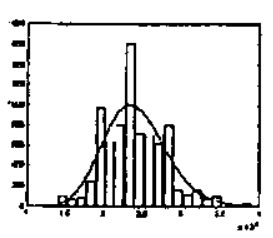

$\lambda_{2}$

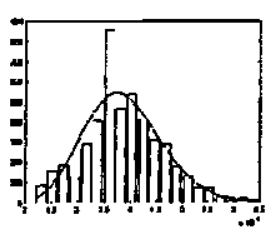

$\lambda_{3}$

Figura 15 - Densidades marginais a posteriori

Na figura 15 apresentamos as densidades marginais a posteriori estimadas por simulação MCMC. Além de não apresentarem simetria algumas dessas densidades têm mais de uma moda.

\section{Interpretação dos resultados:}

Considerando os resultados da tabela $\mathrm{XV}$, temos que a taxa de ocorrência do processo muda de 0.0081 para 0.0024 após 5044 dias e depois muda para $0.0039 \mathrm{em} 18796$ dias. Na figura 12 podemos ver que este resultado é coerente quando assumimos o modelo com dois pontos de mudança. 


\subsubsection{Comparação dos modelos com um e dois pontos}

$\mathrm{Na}$ figura 16 apresentamos o gráfico das densidades preditivas $c_{i}=f\left(x_{i} \mid x_{(i)}\right)$, $i=1,2, \ldots, n$ aproximadas por suas estimativas de Monte Carlo (ver apêndice) para os modelos $M_{1}$ (um único ponto de mudança) e $M_{2}$ (dois ponto de mudança). Observamos que o modelo $M_{2}$ se ajusta melhor aos dados da tabela XIII. Para o modelo $M_{1}$ temos $P_{1}=\prod_{i=1}^{n} \widehat{c}_{1 i}=3.8649 \times 10^{-302} \quad$ e $\quad$ para $\quad$ o modelo $\quad M_{2} \quad$ temos $\quad P_{2}=\prod_{i=1}^{n} \widehat{c}_{2 i}$ $=1.6319 \times 10^{-301}$.

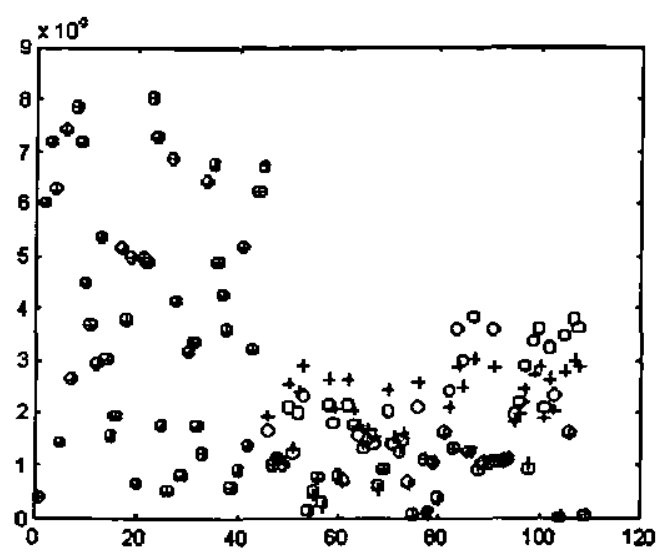

Figura 16 - Gráfico de $c_{i}$ versus $i\left(M_{1}+, M_{2} \mathrm{o}\right)$ 


\section{Conclusões e propostas futuras}

A análise dos modelos estatísticos propostos neste trabalho mostrou que o uso de técnicas Bayesianas para fazer inferência é muito vantajoso com relação às técnicas usuais. O primeiro ponto a favor da inferência Bayesiana é o fato de que através de uma densidade a priori podemos incorporar o conhecimento do especialista no modelo. As técnicas Bayesianas não necessitam de resultados assintóticos, ou seja, podemos resolver problemas nos quais as amostras são pequenas. No caso de problemas com ponto de mudança desconhecido, a estimação por intervalo usando resultados assintóticos não é de simples obtenção. Além disso, quando usamos a abordagem Bayesiana ao calcular intervalos, obtemos um melhor resultado quando o parâmetro têm densidade assimétrica, do que considerando intervalos de confiança baseados na normalidade assintótica dos estimadores de máxima verossimilhança, já que estes assumem simetria na densidade desses estimadores. Um outro fato importante é que os cálculos necessários para obter intervalos de confiança podem se tornar muito dificeis, ou até impraticáveis, à medida que a dimensão dos modelos aumenta. Isso não faz diferença para o cálculo de intervalos de credibiliḍade Bayesianos. A interpretação do intervalo de credibilidade (Bayesiano) é mais fácil já que neste contexto o parâmetro é uma variável aleatória.

Neste trabalho consideramos problemas com ponto de mudança discreto e assumimos que tal ponto só pode ocorrer em um dos valores observados na amostra. Este fato, não permite avaliar a função de verossimilhança em pontos que não estão na amostra $\mathrm{e}$ portanto a estimativa de máxima verossimilhança para o ponto de mudança é imprecisa. Este problema também foi contornado usando inferência Bayesiana.

Também podemos dizer que entre as técnicas Bayesianas estudadas, a simulação de Monte Carlo em cadeias de Markov apresenta a vantagem de ser aplicada mais facilmente do que a técnica de aproximação de integrais de Laplace, sendo que os resultados obtidos são muito semelhantes.

Para dar continuidade à este trabalho podemos considerar o modelo de sobrevivência Weibull com dois tratamentos e com presença de censuras nos dados, usando densidades a priori informativas. Também pode ser feita uma reparametrização deste modelo, visando melhores estimativas por métodos iterativos. Os modelos de sobrevivência apresentados podem ser extendidos para problemas com mais pontos de mudança. No caso do processo de Poisson com pontos de mudanças, uma generalização pode ser feita considerando problemas com taxa de ocorrência variável. 


\section{Apêndice}

\section{Método de aproximação de Laplace :}

Em análise Bayesiana muitas vezes a integração da densidade conjunta a posteriori para obtenção de densidades marginais é muito complex̧a e só pode feita de forma aproximada. O método de aproximação de integrais de Laplace é muito usado nesses casos. Para o uso deste método, a integral a ser aproximada é escrita na forma

$$
I=\int f(\theta) \exp \{-n h(\theta)\} d \theta,
$$

onde $h(\theta)$ é uma função unimodal com máximo em $\theta$ e satisfazendo condições usuais de regularidade. Desenvolvendo $f$ e $h$ em série de Taylor em torno de $\theta$, a aproximação de Laplace para $I$ é dada por:

$$
I \approx\left[\frac{2 \pi}{n}\right]^{1 / 2} \sigma f(\theta) \exp \{-n h(\theta)\}
$$

onde $\sigma=\left\{\left|h^{\prime \prime}(\theta)\right|\right\}^{-1 / 2}$

Uma vez obtidas as aproximações para as densidades marginais a posteriori, o cálculo das médias a posteriori pode ser também aproximado pelo método de Laplace como segue:

A média a posteriori de $\theta$ é dada por

$E(\theta \backslash \mathcal{D})=\frac{\int \theta \pi(\theta) l(\theta) d \theta}{\int \pi(\theta) l(\theta) d \theta}$

Escrevendo essas integrais na forma (1), temos:

$E(\theta \backslash \mathcal{D})=\frac{\int \exp \left\{-n L^{*}(\theta)\right\} d \theta}{\int \exp \{-n L(\theta)\} d \theta}$,

onde

$$
\begin{aligned}
& -n L(\theta)=\ln \pi(\theta)+\ln l(\theta), \\
& -n L^{*}(\theta)=\ln \theta+\ln \pi(\theta)+\ln l(\theta) .
\end{aligned}
$$


Usando (2) podemos aproximar (3) por:

$E(\theta \backslash \mathcal{D}) \approx\left[\frac{\left|V^{*}\right|}{|V|}\right]^{1 / 2} \exp \left\{-n\left[L(m)-L^{*}\left(m^{*}\right)\right]\right\}$

onde $m$ e $m^{*}$ são as modas de $L$ e $L^{*}$, respectivamente e $V$ e $V^{*}$ são as inversas de $L^{\prime \prime}(m)$ e $L^{* \prime \prime}\left(m^{*}\right)$, respectivamente.

Resultados similares são obtidos para o caso multiparamétrico.

\section{Métodos de Simulação de Monte Carlo em cadeias de Markov}

Aqqui apresentamos dois métodos de simulação de Monte Carlo em cadeias da Markov, no caso, os algoritmos amostrador de Gibbs [ver Gelfand,A.E. e Smith,A.F.M., 1990] e Metropolis-Hastings [ver Chib,S. e Greenberg,E., 1995 ou Smith,A.F.M. e Roberts,G.O., 1993]. Com esses métodos encontramos estimativas pontuais e por intervalo dos parâmetros, inclusive do ponto de mudança do risco.

\section{Algoritmo amostrador de Gibbs:}

O amostrador de Gibbs é um esquema iterativo de amostragem de uma cadeia de Markov até obtenção de uma distribuição estacionária.

Supor que da distribuição de interesse $\pi(\theta)$, onde $\theta=\left(\theta_{1}, \ldots, \theta_{p}\right)$, seja muito dificil ou impossível gerar amostras diretamente, mas as densidades a posteriori condicionais $\pi_{i}\left(\theta_{i}\right)=\pi\left(\theta_{i} \backslash \theta_{-i}\right)$ onde $\theta_{-i}=\left(\theta_{1}, \theta_{2}, \ldots, \theta_{i-1}, \theta_{i+1}, \ldots, \theta_{p}\right), i=1, \ldots, p$, são disponíveis e delas podemos gerar amostras facilmente. Com gerações sucessivas dessas densidades condicionais obtemos uma amostra de $\pi(\theta)$ da seguinte forma:

1) Escolher valores iniciais para $\theta, \theta^{(0)}=\left(\theta_{1}^{(0)}, \ldots, \theta_{p}^{(0)}\right)$;

2) Obter novos valores $\theta^{(\mathrm{k})}=\left(\theta_{1}^{(\mathrm{k})}, \ldots, \theta_{\mathbf{p}}^{(\mathrm{k})}\right)$ a partir de $\theta^{(\mathbf{k}-1)}$ através de sucessivas gerações de valores.

$$
\begin{aligned}
& \theta_{1}^{(k)} \sim \pi\left(\theta_{1} \backslash \theta_{2}^{(k-1)}, \theta_{3}^{(k-1)}, \theta_{4}^{(k-1)}, \ldots, \theta_{p}^{(k-1)}\right) \\
& \theta_{2}^{(k)} \sim \pi\left(\theta_{2} \backslash \theta_{1}^{(k)}, \theta_{3}^{(k-1)}, \theta_{4}^{(k-1)}, \ldots, \theta_{p}^{(k-1)}\right) \\
& \ldots \\
& \theta_{p}^{(k)} \sim \pi\left(\theta_{p} \backslash \theta_{1}^{(k)}, \theta_{2}^{(k)}, \theta_{3}^{(k)}, \theta_{4}^{(k)}, \ldots, \theta_{p-1}^{(k)}\right)
\end{aligned}
$$

3) Repetir até obter uma distribuição estacionária. 


\section{Algoritmo Metropolis-Hastings}

Em muitas aplicações, não temos formas conhecidas nas distribuições condicionais usadas no algoritmo amostrador de Gibbs. Nesse caso, usamos o algoritmo MetropolisHastings. Nesse algoritmo, se no estágio $k$ a cadeia está no estado $x$, o próximo estado da cadeia é encontrado gerando um valor candidato $y$ de uma densidade arbitrária $q(x, y)$. Se esta densidade satisfaz a condição de reversibilidade da cadeia, dada por,

$q(x, y) \pi(x)=q(y, x) \pi(y)$

então temos um núcleo de transição satisfatório e todos os valores gerados serão aceitos como no amostrador de Gibbs. No entanto, podemos ter para algum $(x, y)$ que

$q(x, y) \pi(x)>q(y, x) \pi(y)$

Nesse caso, a cadeia se move de $x$ para $y$ com maior frequência. Para corrigir isto, reduzimos o número de movimentos de $x$ para $y$ introduzindo a probabilidade de ocorrer este movimento $\alpha(x, y)<1$. Se o movimento ocorre, então $x^{(k+1)}=y$, caso contrário $x^{(k+1)}=x^{(k)}$.

Assim temos a relação:

$q(x, y) \pi(x) \alpha(x, y)=q(y, x) \pi(y)$

Com isso, a condição de reversibilidade da cadeia é satisfeita por construção e a probabilidade de aceitação do valor gerado é:

$\alpha(x, y)= \begin{cases}\min \left\{\frac{q(y, x) \pi(y)}{q(x, y) \pi(x)}, 1\right\} & \text { se } q(x, y) \pi(x)>0 \\ 1 & \text { caso contrário }\end{cases}$

Em termos práticos, a simulação de uma amostra de $\pi$ por este método pode ser feita da seguinte forma:

1) Escolher um valor inicial para $x, x^{(0)}$;

2) No estágio $k$, mover a cadeia para um novo valor $y$ gerado da densidade $q\left(x^{(k-1)}, y\right)$;

3) Calcular a probabilidade de aceitação do movimento, $\alpha\left(x^{(k-1)}, y\right)$ e gerar $u \sim U[0,1]$. Se o movimento for aceito, $u \leq \alpha\left(x^{(k-1)}, y\right)$, então $x^{(k)}=y$; caso contrário, $x^{(k)}=x^{(k-1)}$ e a cadeia não se move.

4) Repetir esses passos até obter uma distribuição estacionária. 
Uma forma de verificar a convergência do algoritmo é gerar várias cadeias com diferentes condições iniciais para se certificar que se trata de uma cadeia de Markov irredutível, ou seja, que existe uma distribuição de equilíbrio. Existem vários critérios para verificar a convergência dos algoritmos. Neste trabalho vamos usar o critério proposto por Gelman e Rubin [Gelman, A.E. e Rubin,D., 1992].

No caso do algoritmo Metropolis-Hastings também é importante verificar a porcentagem de aceitação dos valores gerados. Quando temos pouca aceitação podemos estar gerando valores com um núcleo que não é adequado, ou seja, é preciso módificar a média e/ou variância do núcleo.

Outro problema no uso do algoritmo Metropolis-Hastings, é que as amostras geradas têm autocorrelação alta e isso implica em um aumento da variância da média amostral, que inclui esta correlação. Para diminuir a autocorrelação é preciso gerar cadeias grandes e então, selecionar sistematicamente valores da cadeia obtendo com isso a amostra final com a qual podemos fazer inferência.

Nesse trabalho, todas as amostras foram obtidas como segue: Foram gerados 10000 valores, sendo 5 cadeias de 2000 valores, cada uma gerada com um valor inicial diferente. Desprezamos os primeiros 500 valores de cada cadeia e selecionamos uma amostra final de tamanho 500, tomando de 15 em 15 dos 1500 valores restantes, ou seja, a amostra final é formada pelos valores das iterações: $515^{\mathrm{a}}, 530^{\mathrm{a}}, 545^{\mathrm{a}}, \ldots, 2000^{\mathrm{a}}$ de cada uma das 5 cadeias.

\section{Critério Bayesiano de seleção de modelos:}

Em análise Bayesiana existem vários métodos para seleção de modelos [Chang, $\mathrm{H}$., 1995]. Um desses métodos é o que utiliza o fator de Bayes. A principal dificuldade do uso do fator de Bayes é a escolha de uma densidade a priori para cada um dos modelos. Quando essa escolha é tal que não dá preferência a um dos modelos, esse método coincide com o teste da razão de verossimilhança, caso contrário, mesmo utilizando densidades a priori não informativas o fator de Bayes pode indicar um modelo inadequado. Por esta razão, optamos pelo uso de densidades preditivas para a seleção de modelos.

\section{Densidades preditivas ordenadas}

A densidade preditiva para $t_{i}$ dado $_{(i)}=\left(t_{1}, t_{2}, \ldots, t_{i-1}, t_{i+1}, \ldots, t_{n}\right)$ é dada por:

$$
c_{i}=f\left(t_{i} \backslash t_{(i)}\right)=\int f\left(t_{i} \backslash \underline{\theta}\right) \pi\left(\underline{\underline{\theta}} \underline{t}_{(i)}\right) d \underline{\theta}, \quad i=1,2, \ldots, n
$$

onde $\pi\left(\theta \backslash t_{(i)}\right)$ é a densidade a posteriori para o vetor de parametros $\underline{\theta}$, dado os dados $\underline{t}_{(i)}$. 
Usando o algoritmo amostrador de Gibbs, podemos aproximar $c_{i}$ usando o estimador de Monte Carlo,

$$
\widehat{c}_{i}=\widehat{f}\left(t_{i} \backslash t_{(i)}\right)=\frac{1}{N} \sum_{j=1}^{N} f\left(t_{i} \backslash \Theta^{(j)}\right)
$$

onde $\underline{\theta}^{(j)}, j=1, \ldots, N$, são as amostras geradas pelo amostrador de Gibbs.

Podemos usar $\widehat{c}_{i}$ para a seleção de modelos. Assim, consideramos gráficos de $\widehat{c}_{i}$ versus $i, i=1, \ldots, n$, para modelos diferentes e o modelo mais adequado para os dados é o que apresenta valores maiores de $\widehat{c}_{i}$ (em média). Também podemos escolher o modelo tal que $c_{l}=\prod_{i=1}^{n} \widehat{c}_{i}(l)$ é máximo, onde $l$ indica o modelo. 


\section{Programas em Matlab}

Programa para o modelo Weibull com um tratamento e um ponto de mudança desconhecido:

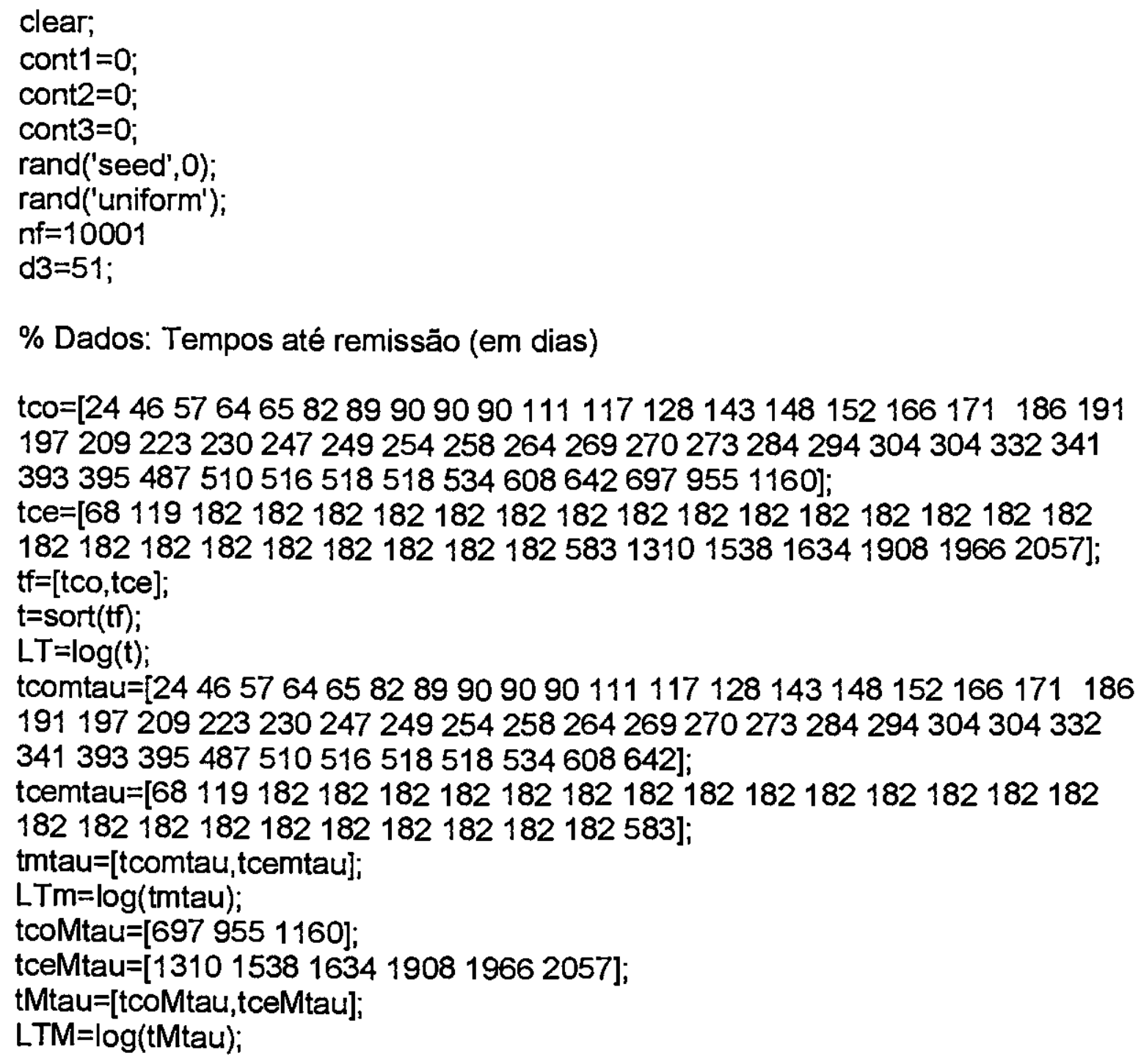

\% Condições iniciais diferentes

$$
\begin{aligned}
& \mathrm{V}=\left[\begin{array}{lllll}
0.0002 & 0.0006 & 0.0005 & 0.0007 & 0.0001
\end{array}\right. \\
& \begin{array}{lllll}
0.8 & 0.25 & 0.3 & 0.6 & 0.15
\end{array} \\
& \begin{array}{lllll}
1.1 & 1.0 & 1.2 & 1.3 & 0.9
\end{array} \\
& \begin{array}{lllll}
600 & 800 & 700 & 600 & 650] ;
\end{array}
\end{aligned}
$$

$$
\begin{aligned}
& \text { for } k=2: n f \\
& \quad \text { if } \operatorname{rem}(k-2,2000)==0 \quad \& \quad k<10000 \\
& \quad \operatorname{lambda}(k-1)=v(1,1+(k-2) / 2000) ;
\end{aligned}
$$




$$
\begin{aligned}
& \text { alfa(k-1)=v(2,1+(k-2)/2000); } \\
& \operatorname{gama}(\mathrm{k}-1)=v(3,1+(\mathrm{k}-2) / 2000) \\
& \operatorname{tau}(\mathrm{k}-1)=\mathrm{v}(4,1+(\mathrm{k}-2) / 2000) \\
& \text { end }
\end{aligned}
$$

\% Geração de tau

$y=$ unifrnd $(100,800)$;

$\%$ Teste $(\mathrm{MH})$ para tau

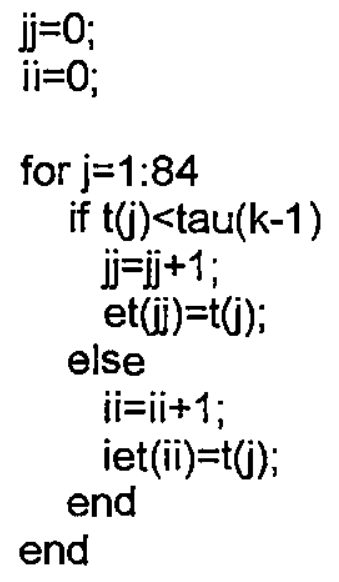

$$
\begin{aligned}
& \mathrm{d} 2 \mathrm{t}(\mathrm{k})=\mathrm{jj} ; \\
& \mathrm{jj}=0 ; \\
& \mathrm{ii}=0 ;
\end{aligned}
$$$$
\text { for } j=1: 51
$$$$
\text { if tco(j)<tau }(k-1)
$$$$
\mathrm{jj}=\mathrm{jj}+1 \text {; }
$$$$
\text { etco(ij)=tco(j); }
$$$$
\text { else }
$$$$
\mathrm{ij}=\mathrm{ij}+1 \text {; }
$$$$
\text { ietco }(i i)=\operatorname{tco}(j) \text {; }
$$$$
\text { end }
$$

end

$\mathrm{d} 1 \mathrm{t}(\mathrm{k})=\mathrm{jj} ;$

s 1 tau $(k)=$ sum(et.^ gama(k-1));

s2tau $(k)=\operatorname{sum}\left(\right.$ iet. ${ }^{\wedge}\left(\right.$ alfa $(k-1)^{\star}$ gama $\left.\left.(k-1)\right)\right)$;

s3tau $(k)=s u m(\log ($ etco $))$;

$\mathrm{s} 4 \mathrm{tau}(\mathrm{k})=\operatorname{sum}(\log ($ ietco $))$;

$\mathrm{r} 1 \mathrm{tau}(\mathrm{k})=\operatorname{tau}(\mathrm{k}-1)^{\wedge} \operatorname{gama}(\mathrm{k}-1)-\operatorname{tau}(\mathrm{k}-1)^{\wedge}\left(\operatorname{alfa}(\mathrm{k}-1)^{\star} \operatorname{gama}(\mathrm{k}-1)\right)$;

$\mathrm{jj}=0$;

$\mathrm{ii}=0$;

for $j=1: 84$ 


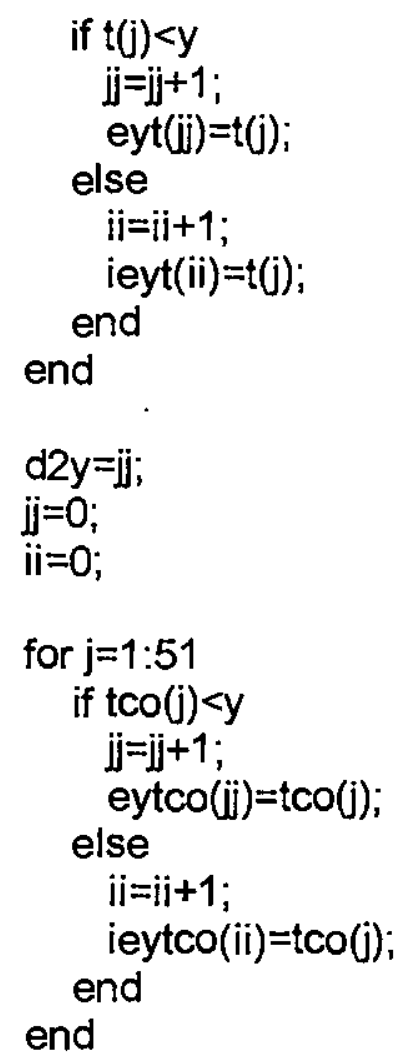

$\mathrm{d} 1 \mathrm{y}=\mathrm{ji}$;

s1y:=sum(eyt.^gama(k-1));

s2y=sum(ieyt.^(alfa(k-1)*gama(k-1)));

s3y=sum(log(eytco));

s4y=sum(log (ieytco));

$r 1 y=y^{\wedge}$ gama(k-1)-y^(alfa(k-1)*gama(k-1));

$p n=\left(\text { alfa }(k-1)^{\wedge}(-d 1 y)\right)^{\star} \exp \left(\right.$ gama $(k-1)^{\star}\left(s 3 y+a l f a(k-1)^{\star} s 4 y\right)-\operatorname{lambda}(k-1)^{\star}(s 1 y+s 2 y-$ d2y*r1y));

$\mathrm{pd}=\left(\mathrm{alfa}(\mathrm{k}-1)^{\wedge}(-\mathrm{d} 1 \mathrm{t}(\mathrm{k}))\right)^{\star} \exp \left(\right.$ gama $(\mathrm{k}-1)^{\star}\left(\mathrm{s} 3 \operatorname{tau}(\mathrm{k})+\mathrm{alfa}(\mathrm{k}-1)^{\star} \mathrm{s} 4 \mathrm{tau}(\mathrm{k})\right)-$ lambda$(k-$ $\left.1)^{\star}\left(\mathrm{s} 1 \operatorname{tau}(k)+s 2 \operatorname{tau}(k)-d 2 t(k)^{\star} r \cdot 1 \operatorname{tau}(k)\right)\right)$;

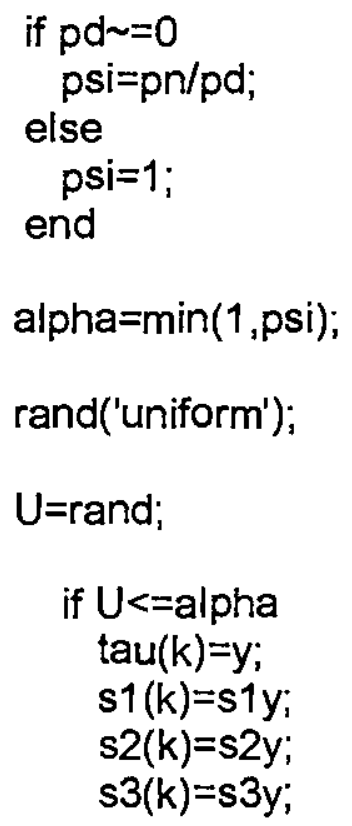




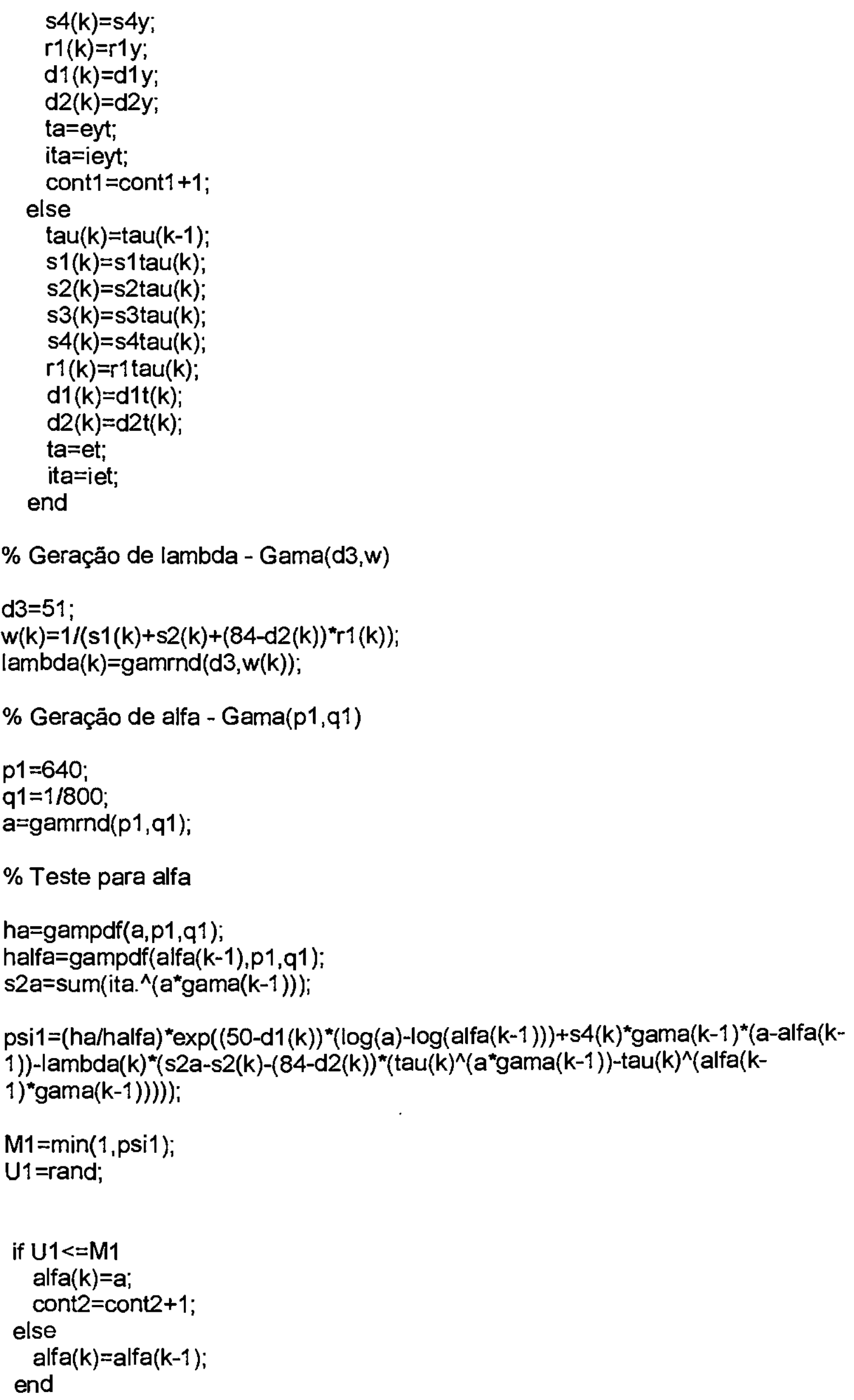


\% Geração de gama - gama(p2,q2)

$\mathrm{p} 2=4431$;

$q 2=1 / 3692 ;$

$g=g a m r n d(p 2, q 2)$;

$\%$ Teste para gama

$h g=g a m p d f(g, p 2, q 2)$;

hgama=gampdf(gama(k-1),p2,q2);

s1g=sum(ta.^g);

s2g=sum(ita. ^(alfa(k)*g));

$\mathrm{r} 1 \mathrm{~g}=\operatorname{tau}(\mathrm{k})^{\wedge} \mathrm{g}-\operatorname{tau}(\mathrm{k})^{\wedge}\left(\mathrm{alfa}(\mathrm{k})^{\star} \mathrm{g}\right)$;

psi2 $=(\text { hg/hgama })^{*} \exp \left(50^{*}(\log (g)-\log (\right.$ gama $(\mathrm{k}-1)))+\left(\mathrm{s} 3(\mathrm{k})+\mathrm{s} 4(\mathrm{k})^{\star} \text { alfa }(\mathrm{k})\right)^{*}(\mathrm{~g}-\mathrm{gama}(\mathrm{k}-$ 1))-lambda(k)*(s1g-s1(k)+s2g-s2(k)+(84-d2(k))*(r1g-r1(k))));

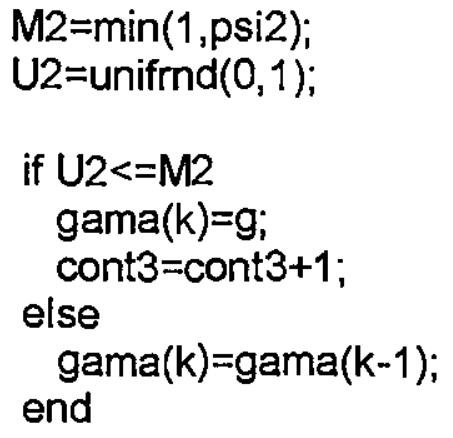

end

\% Seleção das amostras

$\mathrm{k}=1$;

for $\mathrm{j}=501: 2000: 10000$;

for $i=j: j+1499$;

if $\operatorname{rem}(i-j+1,15)==0$

lambdas $(k)=l a m b d a(i)$;

alfas $(k)=a l f a(i)$;

gamas $(k)=g a m a(i)$

$\operatorname{taus}(k)=\operatorname{tau}(\mathrm{i})$;

$\mathrm{k}=\mathrm{k}+1$;

end

end

end

\% Critério de convergência - Gelman e Rubin
$\mathrm{nc}=5$;
$\mathrm{n} 1=1$;
$\mathrm{n} 2=100$;
$d=100$; 
$\%$ Critério de parada para tau

Metau=[mean(taus $(n 1: n 2))$, mean $(\operatorname{taus}(n 1+d: n 2+d))$, mean $\left(\operatorname{taus}\left(n 1+2^{\star} d: n 2+2^{*} d\right)\right), m$ ean(taus $\left.\left(n 1+3^{\star} d: n 2+3^{*} d\right)\right)$, mean(taus $\left.\left(n 1+4^{\star} d: n 2+4^{*} d\right)\right)$;

QMtau=Metau. *Metau;

Vatau $=\left[\operatorname{cov}(\operatorname{taus}(n 1: n 2)), \operatorname{cov}(\operatorname{taus}(n 1+d: n 2+d)), \operatorname{cov}\left(\operatorname{taus}\left(n 1+2^{*} d: n 2+2^{\star} d\right)\right)\right.$, $\left.\operatorname{cov}\left(\operatorname{taus}\left(n 1+3^{*} d: n 2+3^{*} d\right)\right), \operatorname{cov}\left(\operatorname{taus}\left(n 1+4^{*} d: n 2+4^{*} d\right)\right)\right]$;

MeMtau=sum(Metau)/nc;

Wtau=sum(Vatau)/nc;

VarVtau=(Vatau*Vatau'-nc*Wtau^2)/(nc-1);

Utau $=\left(\mathrm{d} /(\text { nc-1) })^{\star}\left(\right.\right.$ Metau ${ }^{*}$ Metau'-nc*MeMtau $\left.{ }^{\wedge} 2\right)$;

VARtau $=((d-1) / d) * W t a u+(1 / d) * U$ tau;

VHtau=VARtau+Utau/(nc*d);

Mcovtau=cov(Vatau,Metau);

Qcovtau=cov(Vatau, QMtau);

COVtau=Qcovtau(1,2)-2*MeMtau*Mcovtau(1,2);

$\operatorname{VarVHtau}=((\mathrm{d}-1) / \mathrm{d})^{\wedge} 2^{\star}(1 / \mathrm{nc})^{\star} \operatorname{VarVtau}+\left(\left((\mathrm{nc}+1) /\left(\mathrm{nc} \mathrm{c}^{*} \mathrm{~d}\right)\right)^{\wedge} 2\right)^{\star}(2 /(\mathrm{nc}-$

1))*Utau^2+2*COVtau* $(n c+1)^{\star}(d-1) /\left(d^{\star} n c^{\wedge} 2\right)$;

dftau $=2^{\star}\left((\text { VHtau })^{\wedge} 2\right)$ NarVHtau;

Rtau=sqrt $\left((\text { VHtau} / \text { Wtau })^{\star}\right.$ dftau/(dftau-2))

$\%$ Critério de parada para lambda

MeL $=[$ mean(lambdas $(n 1: n 2))$, mean(lambdas $(n 1+d: n 2+d))$, mean(lambdas $\left(n 1+2^{*} d\right.$ : $\left.n 2+2^{*} d\right)$ ), mean(lambdas $\left(n 1+3^{*} d: n 2+3^{*} d\right)$ ), mean(lambdas $\left.\left.\left(n 1+4^{*} d: n 2+4^{*} d\right)\right)\right]$;

$\mathrm{QML}=\mathrm{MeL} .{ }^{*} \mathrm{MeL}$;

$\operatorname{VaL}=[\operatorname{cov}(\operatorname{lambdas}(n 1: n 2)), \operatorname{cov}($ lambdas $(n 1+\mathrm{d}: n 2+\mathrm{d}))$, $\operatorname{cov}\left(\operatorname{lambdas}\left(n 1+2^{\star} d: n 2+2^{\star} d\right)\right)$, cov $\left(\right.$ lambdas $\left.\left(n 1+3^{\star} d: n 2+3^{*} d\right)\right)$, cov $\left(\right.$ lambdas $\left.\left(n 1+4^{*} d: n 2+4^{*} d\right)\right)$;

MeML=sum(MeL)/nc;

$W L=\operatorname{sum}(\mathrm{VaL}) / \mathrm{nc} ;$

$\operatorname{VarVL}=\left(\operatorname{VaL}^{*} \operatorname{VaL}^{\prime}-\mathrm{nc}^{*} \mathrm{WL}^{\wedge} 2\right) /(\mathrm{nc}-1)$;

$U L=(d /(n c-1))^{*}\left(M^{\prime} L^{*} M e L^{\prime}-n^{*} M e M L \wedge 2\right)$;

VARL $=((d-1) / d)^{*} W L+(1 / d)^{*} U L$;

$V H L=V A R L+U L /\left(n c^{*} d\right)$;

McovL=cov $(\mathrm{VaL}, \mathrm{MeL}) ;$

QcovL=cov(VaL, QML);

$\operatorname{COVL}=\mathrm{Q} \operatorname{covL}(1,2)-2^{*} \mathrm{MeML}{ }^{*} \operatorname{McovL}(1,2)$;

$\operatorname{Var} V H L=((d-1) / d)^{\wedge} 2^{*}(1 / n c)^{*} \operatorname{VarVL}+\left(\left((n c+1) /\left(n c^{*} d\right)\right)^{\wedge} 2\right)^{*}(2 /(n c-$

1)) $\mathrm{UL}^{\wedge} 2+2^{*} \mathrm{COVL}(\mathrm{nc}+1)^{\star}(\mathrm{d}-1) /\left(\mathrm{d}^{\star} n \mathrm{c}^{\wedge} 2\right)$;

$\mathrm{dfL}=2^{*}\left((\mathrm{VHL})^{\wedge} 2\right) / \mathrm{NarVHL;}$

$R L=s q r t\left((V H L W L)^{*} \mathrm{dfL} /(\mathrm{dfL}-2)\right)$

$\%$ Critério de parada para alfa

Mealfa $=[$ mean(alfas(n1:n2)), mean(alfas $(n 1+d: n 2+d))$, mean(alfas $\left.\left(n 1+2^{*} d: n 2+2^{*} d\right)\right)$ ,mean(alfas $\left(n 1+3^{*} d: n 2+3^{*} d\right)$ ), mean(alfas $\left.\left(n 1+4^{*} d: n 2+4^{*} d\right)\right)$;

QMalfa $=$ Mealfa. ${ }^{*}$ Mealfa;

Vaalfa $=\left[\operatorname{cov}(\operatorname{alfas}(n 1: n 2)), \operatorname{cov}(\right.$ alfas $(n 1+d: n 2+d)), \operatorname{cov}\left(\right.$ alfas $\left.\left(n 1+2^{*} d: n 2+2^{*} d\right)\right)$, $\operatorname{cov}\left(\right.$ alfas $\left.\left.\left(n 1+3^{*} d: n 2+3^{*} d\right)\right), \operatorname{cov}\left(\operatorname{alfas}\left(n 1+4^{*} d: n 2+4^{*} d\right)\right)\right]$;

MeMalfa=sum(Mealfa)/nc;

Walfa $=\operatorname{sum}($ Vaalfa $) / n c$; 
VarValfa=(Vaalfa* Vaalfa'-nc* Walfa^2)/(nc-1);

Ualfa $=d^{\star}($ Mealfa*Mealfa'-nc*MeMalfa^2)/(nc-1);

VARalfa $=((d-1) / d) \star W a l f a+(1 / d) \star U a l f a ;$

VHalfa=VARalfa+Ualfa/(nc*d);

Mcovalfa=cov(Vaalfa,Mealfa);

Qcovalfa=cov(Vaalfa, QMalfa);

COValfa=Qcovalfa(1,2)-2*MeMalfa*Mcovalfa(1,2);

VarVHalfa $=((d-1) / d)^{\wedge} 2^{\star}(1 / n c)^{\star} \operatorname{VarValfa}+\left(\left((n c+1) /\left(n c^{\star} d\right)\right)^{\wedge} 2\right)^{\star}(2 /(n c-$

1)) ${ }^{\star}$ Ualfa $^{\wedge} 2+2^{\star}$ COValfa* $^{\star}(n c+1)^{\star}(d-1) /\left(d^{\star} n c^{\wedge} 2\right)$;

dfalfa $=2 *($ VHalfa^2) NarVHalfa;

RA=sqrt((VHalfa/ Walfa)*dfalfa/(dfalfa-2));

\% Critério de parada para gama

MeG1 = [mean(gamas(n1:n2)), mean(gamas $(n 1+d: n 2+d))$, mean(gamas $\left(n 1+2^{*} d: n 2+\right.$ $\left.2^{*} d\right)$ ), mean(gamas $\left(n 1+3^{\star} d: n 2+3^{*} d\right)$, mean(gamas $\left.\left(n 1+4^{\star} d: n 2+4^{*} d\right)\right)$;

QMG1=MeG1. $M e G 1$;

VaG1 $=[\operatorname{cov}(g a m a s(n 1: n 2)), \operatorname{cov}($ gamas $(n 1+d: n 2+d))$,

$\operatorname{cov}\left(\right.$ gamas $\left.\left(n 1+2^{\star} d: n 2+2^{*} d\right)\right)$, $\operatorname{cov}\left(\right.$ gamas $\left.\left(n 1+3^{*} d: n 2+3^{*} d\right)\right)$,

$\operatorname{cov}\left(\right.$ gamas $\left.\left.\left(n 1+4^{\star} d: n 2+4^{*} d\right)\right)\right]$;

MeMG1 =sum(MeG1)/nc;

WG1 =sum(VaG1)/nc;

VarVG1=(VaG1 *VaG1'-nc*WG1^2)/(nc-1);

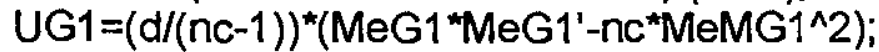

VARG $1=((d-1) / d)^{*} W G 1+(1 / d)^{*} U G 1$;

VHG1 =VARG1 +UG1/(nc*d);

McovG1 =cov $(\operatorname{VaG} 1, \mathrm{MeG} 1)$;

QcovG1=cov $(\operatorname{VaG1} 1, \mathrm{QMG1})$;

COVG1 = QcovG1 $(1,2)-2{ }^{*} M e M G 1 * M \operatorname{McovG} 1(1,2)$;

$\operatorname{VarVHG1}=((\mathrm{d}-1) / \mathrm{d})^{\wedge} 2^{\star}(1 / \mathrm{nc})^{\star} \operatorname{VarVG} 1+\left(\left((\mathrm{nc}+1) /\left(\mathrm{nc} \mathrm{c}^{\star} \mathrm{d}\right)\right)^{\wedge} 2\right)^{\star}(2 /(\mathrm{nc}-$

1) ) UG1^2+2*COVG1 * $(\mathrm{nc}+1)^{\star}(\mathrm{d}-1) /\left(\mathrm{d}^{\star} \mathrm{nc} \mathrm{c}^{\wedge} 2\right)$;

dfG1 =2*((VHG1)^2)NarVHG1;

$\mathrm{RG}=\operatorname{sqr}\left((\mathrm{VHG} 1 \mathrm{WG} 1)^{\star} \mathrm{dfG} 1 /(\mathrm{dfG} 1-2)\right)$

$\%$ Resultados

$T=[$ mean(taus) std(taus) $]$

$L=[$ mean(lambdas) std(lambdas)]

$A=[$ mean(alfas) std(alfas)]

$\mathrm{G}=[$ mean(gamas) std(gamas)]

\% Porcentagem de aceitação

Ctau $=$ cont $1 / 10000$

Calfa $=$ cont $2 / 10000$

Cgama $=$ cont $3 / 10000$

\% Gráficos

hist(taus)

[motau,y]=ginput 
pause

hist(lambdas)

[molam,y]=ginput

pause

hist(alfas)

[moralfa,y]=ginput

hist(gamas)

[mogam, $y$ ] $=$ ginput

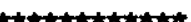

Programa para o processo de Poisson com dois pontos de mudança desconhecidos:

clear

\% Dados: y são os intervalos de tempo entre ocorrências

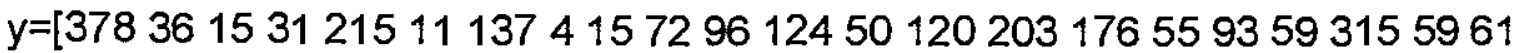
113189345208128611410818823328226178993262755421711332 2315136131235458275781712056444678714812345749849131182 255195224566390722282712085171613543261312348745217120 275206629143693383361932933031217114575364371915647129 1630292177181357 ;

$\mathrm{tt}(1)=378$;

for $i=2: 109$

end

$t t(i)=t t(i-1)+y(i)$;

$\operatorname{cont} 1=0$

cont2 $=0$;

$n=109$

$m=10001$

NTau1(1) $=45$;

NTau2(1) $=35$;

NTau3(1)=n-NTau1 (1)-NTau2(1);

\% Condições iniciais

$\mathrm{V}=\left[\begin{array}{lllll}5376 & 5300 & 5800 & 5400 & 5500\end{array}\right.$

1800017200187001850019000

0.00850 .00700 .00500 .00750 .0084

0.00480 .00450 .0030 .00480 .005

$\begin{array}{lllll}0.0029 & 0.0030 & 0.0051 & 0.0028 & 0.0032]\end{array}$ 
for $k=2: m$

if rem(k-2,2000)==0 \& $k<10000$

tau1 $(\mathrm{k}-1)=\mathrm{v}(1,1+(\mathrm{k}-2) / 2000)$;

$\operatorname{tau} 2(\mathrm{k}-1)=\mathrm{v}(2,1+(\mathrm{k}-2) / 2000)$;

$11(\mathrm{k}-1)=\mathrm{v}(3,1+(\mathrm{k}-2) / 2000)$;

$12(\mathrm{k}-1)=\mathrm{v}(4,1+(\mathrm{k}-2) / 2000)$;

$13(\mathrm{k}-1)=\mathrm{v}(5,1+(\mathrm{k}-2) / 2000)$;

end

$r 1=\operatorname{tau} 1(\mathrm{k}-1)$;

r2=tau2(k-1)-tau1(k-1);

r3=sum(y)-tau2(k-1);

$I 1(k)=\operatorname{gamrnd}(N T a u 1(k-1), 1 / r 1)$;

I2(k)=gamrnd(NTau2(k-1),1/r2);

$13(k)=$ gamrnd $(N T a u 3(k-1), 1 / r 3)$;

$\mathrm{t} 1=$ unifrnd $(5000,5500)$;

t2=unifrnd $(18500,19000)$;

\% Cálculos para os testes $(\mathrm{MH})$ para tau1 e tau2

for $j=1: 109$

$$
\begin{aligned}
& \text { if } \operatorname{tt}(j)<t 1 \\
& \text { et } 1(j)=1 ; \\
& \text { else } \\
& \text { et } 1(j)=0 ; \\
& \text { end }
\end{aligned}
$$

if $\mathrm{tt}(\mathrm{j})>\mathrm{t} 1 \& \mathrm{tt}(\mathrm{j})<\mathrm{t} 2$

$$
\text { et } 2(j)=1 \text {; }
$$

else

$$
\text { et2 }(j)=0 \text {; }
$$

end

end

Nt1 =sum(et1);

$\mathrm{Nt2}=\operatorname{sum}($ et2);

$p \sin 1=\exp \left(-t^{*}(\mid 1(k)-12(k))\right)^{\star}\left(11(k)^{\wedge} \mathrm{Nt1}\right)$;

psid1 $=\exp \left(-\operatorname{tau} 1(\mathrm{k}-1)^{\star}(11(\mathrm{k})-\mathrm{l} 2(\mathrm{k}))\right)^{\star}\left(11(\mathrm{k})^{\wedge} \mathrm{NTau} 1(\mathrm{k}-1)\right)$;

psin $2=\exp \left(-\mathrm{t} 2^{\star}(12(\mathrm{k})-13(\mathrm{k}))\right)^{\star}\left(12(\mathrm{k})^{\wedge} \mathrm{Nt2}\right)$;

psid2 $=\exp \left(-\operatorname{tau} 2(k-1)^{\star}(12(k)-13(k))\right)^{\star}\left(12(k)^{\wedge} N T a u 2(k-1)\right)$; 
$\%$ teste para tau1

if psid1 $=0$

psi1=psin1/psid1;

else

psi1=1;

end

alpha1 $=\min (1, p s i 1)$;

rand('uniform');

U1=rand;

if $\mathrm{U} 1<=$ alpha1

$\operatorname{tau} 1(k)=t 1$;

cont $1=$ cont $1+1$;

NTau1 $(k)=N t 1$;

else

$\operatorname{tau} 1(k)=\operatorname{tau} 1(k-1)$;

NTau1(k)=NTau1(k-1);

end

$\%$ Teste para tau2

if psid2 $=0$

psi2=psin2/psid2;

else

psi2=1;

end

alpha2=min(1,psi2);

rand('uniform');

$\mathrm{U} 2=$ rand;

if $\mathrm{U} 2<=$ alpha2

$\operatorname{tau} 2(k)=t 2$;

cont $2=$ cont $2+1$;

NTau2 $(k)=N+2$;

else

$\operatorname{tau} 2(k)=\operatorname{tau} 2(k-1)$;

NTau2 $(k)=N T a u 2(k-1)$;

end

end

$\mathrm{NTau} 3(\mathrm{k})=\max (0, \mathrm{n}-\mathrm{N} t 1-\mathrm{Nt} 2)$; 
\% Seleção das amostras

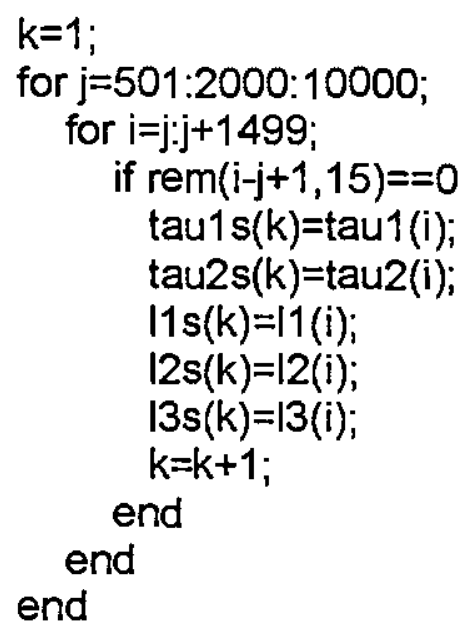

\% Critério de convergência - Gelman e Rubin

$\mathrm{nc}=5$

$\mathrm{n} 1=1$

$\mathrm{n} 2=100$;

$d=100$;

$\%$ Critério de parada para tau1

Metau1 $=\left[\operatorname{mean}(\operatorname{tau} 1 s(n 1: n 2))\right.$, mean $(\operatorname{tau} 1 s(n 1+d: n 2+d))$, mean $\left(\operatorname{tau} 1 s\left(n 1+2^{*} d: n 2+2^{*}\right.\right.$ d)), mean(tau1s $\left.\left(n 1+3^{*} d: n 2+3^{*} d\right)\right)$, mean(tau1s $\left.\left.\left(n 1+4^{*} d: n 2+4^{*} d\right)\right)\right]$;

QMtau1=Metau1. *Metau1;

Vatau1 $=\left[\operatorname{cov}(\operatorname{tau} 1 s(n 1: n 2)), \operatorname{cov}(\operatorname{tau} 1 s(n 1+d: n 2+d)), \operatorname{cov}\left(\operatorname{tau} 1 s\left(n 1+2^{*} d: n 2+2^{*} d\right)\right)\right.$, $\left.\operatorname{cov}\left(\operatorname{tau} 1 s\left(n 1+3^{*} d: n 2+3^{*} d\right)\right), \operatorname{cov}\left(\operatorname{tau} 1 s\left(n 1+4^{*} d: n 2+4^{*} d\right)\right)\right]$;

MeMtau1=sum(Metau1)/nc;

Wtau1=sum(Vatau1)/nc;

VarVtau1=(Vatau1 Vatau1'-nc*Wtau1^2)/(nc-1);

Utau1=(d/(nc-1) $)^{\star}($ Metau1*Metau1'-nc*MeMtau1^2);

VARtau $1=((\mathrm{d}-1) / \mathrm{d}) \star$ Wtau $1+(1 / \mathrm{d})^{\star}$ Utau1;

VHtau1 =VARtau1+Utau1/(nc*d);

Mcovtau1=cov(Vatau1, Metau1);

Qcovtau1=cov(Vatau1, QMtau1);

COVtau1=Qcovtau1 $(1,2)-2^{\star}$ MeMtau1 *Mcovtau1(1,2);

VarVHtau1 $=((\mathrm{d}-1) / \mathrm{d})^{\wedge} 2^{\star}(1 / \mathrm{nc})^{\star} \operatorname{VarVtau} 1+\left(\left((\mathrm{nc}+1) /\left(\mathrm{nc}^{\star} \mathrm{d}\right)\right)^{\wedge} 2\right)^{\star}(2 /(\mathrm{nc}-$

1) $)^{\star} \operatorname{Utau}^{\wedge} 2+2^{*} \operatorname{COVtau}^{*}(\mathrm{nc}+1)^{\star}(\mathrm{d}-1) /\left(\mathrm{d}^{\star} \mathrm{nc} \mathrm{c}^{\wedge} 2\right)$;

dftau1=2*((VHtau1)^2)NarVHtau1;

Rtau1=sqrt ((VHtau1/Wtau1)*dftau1/(dftau1-2))

\% Critério de parada para tau2

Metau2 $=\left[\operatorname{mean}(\operatorname{tau} 2 s(n 1: n 2))\right.$, mean $(\operatorname{tau} 2 s(n 1+d: n 2+d))$, mean $\left(\operatorname{tau} 2 s\left(n 1+2^{*} d: n 2+2^{*}\right.\right.$ d)), mean(tau2s $\left.\left(n 1+3^{*} d: n 2+3^{\star} d\right)\right)$, mean(tau2s $\left.\left.\left(n 1+4^{*} d: n 2+4^{*} d\right)\right)\right]$;

QMtau2=Metau2. *Metau2; 
Vatau2 $=\left[\operatorname{cov}(\operatorname{tau} 2 s(n 1: n 2)), \operatorname{cov}(\operatorname{tau} 2 s(n 1+d: n 2+d)), \operatorname{cov}\left(\operatorname{tau} 2 s\left(n 1+2^{*} d: n 2+2^{*} d\right)\right)\right.$, $\left.\operatorname{cov}\left(\operatorname{tau} 2 s\left(n 1+3^{*} d: n 2+3^{*} d\right)\right), \operatorname{cov}\left(\operatorname{tau} 2 s\left(n 1+4^{\star} d: n 2+4^{\star} d\right)\right)\right]$

MeMtau2=sum(Metau2)/nc;

Wtau2=sum(Vatau2)/nc;

VarVtau2=(Vatau2*Vatau2'-nc*Wtau2^2)/(nc-1);

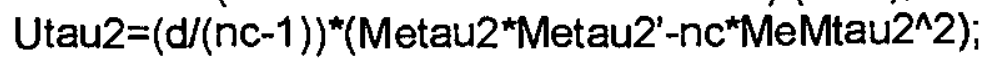

VARtau2 $=((\mathrm{d}-1) / \mathrm{d}){ }^{*}$ Wtau2 $+(1 / \mathrm{d})^{\star} \mathrm{Utau} 2 ;$

VHtau2=VARtau2+Utau2/(nc*d);

Mcovtau2=cov(Vatau2, Metau2);

Qcovtau2=cov(Vatau2, QMtau2);

COVtau2=Qcovtau2 $(1,2)-2 *$ MeMtau2*Mcovtau2 $(1,2)$;

$\operatorname{VarVHtau} 2=((d-1) / d)^{\wedge} 2^{*}(1 / n c)^{\star} \operatorname{VarVtau} 2+\left(\left((n c+1) /\left(n c^{*} d\right)\right)^{\wedge} 2\right)^{\star}(2 /(n c-$

1))*Utau2^ $2+2^{*}$ COVtau2* $(n c+1)^{*}(d-1) /\left(d^{\star} n c^{\wedge} 2\right)$;

dftau2 $=2^{*}\left((\text { VHtau2 })^{\wedge} 2\right)$ NarVHtau2;

Rtau2=sqrt ((VHtau2N'Wtau2)*dftau2/(dftau2-2))

$\%$ Critério de parada para Lambda 1

MeL $1=\left[\operatorname{mean}(11 s(n 1: n 2))\right.$, mean $(11 s(n 1+d: n 2+d))$, mean $\left(11 s\left(n 1+2^{*} d: n 2+2^{*} d\right)\right)$, mean ( $\left.11 s\left(n 1+3^{*} d: n 2+3^{*} d\right)\right)$, mean $\left.\left(11 s\left(n 1+4^{*} d: n 2+4^{*} d\right)\right)\right]$;

QML1=MeL1. MeL1;

$\operatorname{VaL} 1=\left[\operatorname{cov}(11 s(n 1: n 2)), \operatorname{cov}(11 s(n 1+d: n 2+d)), \operatorname{cov}\left(11 s\left(n 1+2^{*} d: n 2+2^{*} d\right)\right)\right.$,

$\left.\operatorname{cov}\left(11 s\left(n 1+3^{*} d: n 2+3^{*} d\right)\right), \operatorname{cov}\left(11 s\left(n 1+4^{*} d: n 2+4^{*} d\right)\right)\right] ;$

MeML1 =sum(MeL1)/nc;

WL1 =sum(VaL1)/nc;

$\operatorname{VarVL1}=\left(\operatorname{VaL} 1^{*} \operatorname{VaL} 1 '-n c^{*} W L 1^{\wedge} 2\right) /(n c-1) ;$

$\mathrm{UL} 1=(\mathrm{d} /(\mathrm{nc}-1))^{\star}\left(\mathrm{MeL} 1^{\star}{ }^{*} \mathrm{MeL} 1^{\prime}-\mathrm{nc} \mathrm{M}^{\star} \mathrm{MeML}{ }^{\wedge} 2\right) ;$

VARL $1=((d-1) / d)^{*} W L 1+(1 / d)^{*} U L 1$;

VHL1=VARL1+UL1/(nc*d);

$\operatorname{McovL} 1=\operatorname{cov}(\operatorname{VaL} 1, \operatorname{MeL} 1)$;

QcovL1= $\operatorname{cov}(\operatorname{VaL} 1, \mathrm{QML} 1)$;

COVL1=QcovL1(1,2)-2*MeML1*McovL1(1,2);

$\operatorname{VarVHL1}=((d-1) / d)^{\wedge} 2^{*}(1 / n c)^{\star} \operatorname{VarVL} 1+\left(\left((n c+1) /\left(n c^{*} d\right)\right)^{\wedge} 2\right)^{\star}(2 /(n c-$

1))*UL1^2+2*COVL1*(nc+1)*(d-1)/(d*nc^2);

dfL1=2*((VHL1)^2) NarVHL1;

RL1=sqrt((VHL1ML1)*dfL1/(dfL1-2))

$\%$ Critério de parada para Lambda 2

MeL2 $=\left[\operatorname{mean}(12 s(n 1: n 2))\right.$, mean $(12 s(n 1+d: n 2+d))$, mean $\left(12 s\left(n 1+2^{*} d: n 2+2^{*} d\right)\right)$, mean( $\left.12 s\left(n 1+3^{*} d: n 2+3^{*} d\right)\right)$, mean $\left.\left(12 s\left(n 1+4^{*} d: n 2+4^{*} d\right)\right)\right]$;

QML.2=MeL2. MeL2;

$\operatorname{VaL2}=\left[\operatorname{cov}(12 s(n 1: n 2)), \operatorname{cov}(12 \operatorname{s}(n 1+d: n 2+d)), \operatorname{cov}\left(12 s\left(n 1+2^{*} d: n 2+2^{*} d\right)\right)\right.$,

$\left.\operatorname{cov}\left(12 s\left(n 1+3^{*} d: n 2+3^{*} d\right)\right), \operatorname{cov}\left(12 s\left(n 1+4^{*} d: n 2+4^{*} d\right)\right)\right] ;$

MeML2=sum(MeL2)/nc;

WL2=sum(VaL2)/nc;

$\operatorname{VarVL2}=\left(\operatorname{VaL} 2 * \operatorname{VaL2}-\mathrm{nc}^{*} \mathrm{WL} 2^{\wedge} 2\right) /(\mathrm{nc}-1)$;

UL2 $=(d /(n c-1))^{*}($ MeL2*MeL2'-nc*MeML2^2);

VARL2 $=((d-1) / d)^{*} W L 2+(1 / d) * U L 2 ;$

VHL2=VARL2+UL2/(nc*d);

McovL2=cov(VaL2, MeL2);

QcovL2=cov(VaL2, QML2); 
COVL2=QcovL2(1,2)-2*MeML2*McovL2(1,2);

$\operatorname{VarVHL} 2=((\mathrm{d}-1) / \mathrm{d})^{\wedge} 2^{\star}(1 / \mathrm{nc})^{\star} \operatorname{VarVL} 2+\left(\left((\mathrm{nc}+1) /\left(\mathrm{nc} \mathrm{c}^{*}\right)\right)^{\wedge} 2\right)^{\star}(2 /(\mathrm{nc}-$

1) $)^{\star} U L 2^{\wedge} 2+2^{\star} \operatorname{COVL2}{ }^{\star}(n c+1)^{\star}(d-1) /\left(d^{\star} n c^{\wedge} 2\right) ;$

dfL2=2*((VHL2)^2) NarVHL2;

RL2=sqrt((VHL2NL2)*dfL2/(dfL2-2))

\% Critério de parada para Lambda3

MeL3=[mean $(13 s(n 1: n 2))$, mean $(13 s(n 1+d: n 2+d))$, mean $\left(13 s\left(n 1+2^{*} d: n 2+2^{*} d\right)\right)$, mean ( $\left.13 s\left(n 1+3^{*} d: n 2+3^{*} d\right)\right)$, mean $\left.\left(13 s\left(n 1+4^{*} d: n 2+4^{*} d\right)\right)\right]$;

QML3=MeL3. ${ }^{*} \mathrm{MeL} 3$;

Val.3 $=\left[\operatorname{cov}(13 s(n 1: n 2)), \operatorname{cov}(13 s(n 1+d: n 2+d)), \operatorname{cov}\left(13 s\left(n 1+2^{*} d: n 2+2^{*} d\right)\right)\right.$, $\operatorname{cov}\left(13 s\left(n 1+3^{\star} d: n 2+3^{\star} d\right)\right), \operatorname{cov}\left(\left[3 s\left(n 1+4^{*} d: n 2+4^{*} d\right)\right)\right]$

MeML3=sum(MeL3)/nc;

WL3=sum(VaL3)/nc;

VarVL3=(VaL_3*VaL3'-nc*WL3^2)/(nc-1);

$\mathrm{UL3}=(\mathrm{d} /(\mathrm{nc}-1))^{\star}\left(\mathrm{MeL}^{\star}{ }^{\star} \mathrm{MeL} 3^{\prime}-\mathrm{nc}^{\star} \mathrm{MeML} 3^{\wedge} 2\right)$;

VARL3=((d-1)/d $)^{*}$ WL.3+(1/d $)^{*}$ UL3;

VHL3=VARL3+UL3/(nc*d);

McovL3=cov $(\mathrm{VaL} 3, \mathrm{MeL} 3)$;

QcovL3=cov(VaL3,QML3);

COVL3=QcovL3(1,2)-2*MeML3*McovL3(1,2);

$\operatorname{VarVHL3}=((\mathrm{d}-1) / \mathrm{d})^{\wedge} 2^{*}(1 / \mathrm{nc})^{\star} \operatorname{VarVL} 3+\left(\left((\mathrm{nc}+1) /\left(\mathrm{nc}{ }^{*} \mathrm{~d}\right)\right)^{\wedge} 2\right)^{\star}(2 /(\mathrm{nc}-$

1) $)^{\star} U L 3^{\wedge} 2+2^{*} \operatorname{COVL}^{\star}(n c+1)^{\star}(d-1) /\left(d^{\star} n c^{\wedge} 2\right)$;

dfL3 $=2^{*}\left((\text { VHL3 })^{\wedge} 2\right)$ NarVHL3;

RL3=sqrt((VHL3/WL3)*dfL3/(dfL3-2))

$\%$ Resultados

$\mathrm{T} 1=[\mathrm{mean}(\operatorname{tau} 1 \mathrm{~s}) \mathrm{std}(\operatorname{tau} 1 \mathrm{~s})]$

$\mathrm{T} 2=[\operatorname{mean}(\operatorname{tau} 2 \mathrm{~s}) \mathrm{std}(\operatorname{tau} 2 \mathrm{~s})]$

$L 1=[$ mean $(11 \mathrm{~s}) \mathrm{std}(11 \mathrm{~s})]$

$L 2=[\operatorname{mean}(12 \mathrm{~s}) \mathrm{std}(12 \mathrm{~s})]$

$\mathrm{L} 3=[$ mean $(13 \mathrm{~s}) \mathrm{std}(13 \mathrm{~s})]$

\% Porcentagem de aceitação

CT1 $=$ cont $1 / 10000$

CT2 $=$ cont $2 / 10000$

$\%$ Gráficos

hist(tau1s)

[mot $1, y]=$ ginput

hist(tau2s)

[mot2,y]=ginput

hist(11s)

$[\mathrm{mol} 1, y]=$ ginput

hist(I2s)

[mol2,y]=ginput

hist(13s)

$[\mathrm{mol} 3, y]=$ giput 


\section{Referências:}

Achcar, J. A. ; Andrade, M. G. ; Loibel, S. M. C. (1998), Weibull hazard function with a change-point: A Bayesian approach using Markov chain Monte Carlo methods. Journal of Statistical Research (EUA), aceito para publicação.

Achcar, J.A. ; Bolfarine, H. (1989), Constant hazard against a change-point alternative: a Bayesian approach with censored data. Communications in Statistics - Theory and methods, 18(10), 3801-3819.

Achcar, J. A. ; Loibel, S. M. C. (1998), Constant Hazard Function Models with a change point: A Bayesian Analysis using Markov Chain Monte Carlo Methods. Biometrical Journal (Magdeburg, Alemanha), aceito para publicação.

Box, G.E. ; Tiao, G.C. (1973). Bayesian inference in Statistical Analysis, New York: Addison-Wesley.

Carlin, B. P. ; Gelfand, A. E. ; Smith, A. F. M. (1992), Hierarchical Bayesian Analysis of changepoint problems. Appl. Statist. , 41(2), 389-405.

Chang, H. (1995), Model determinations using predictive distributions. phD thesis, Department of statistics - University of Connecticut.

Chib,S. ; Greenberg, E. (1995). Understanding the Metropolis-Hastings algorithm. American Statistician, 49,4,327-335.

Christensen, J. ; Rudemo, M. (1996), Multiple change-point analysis of disease incidence rates. Preventive Veterinary Medicine, 26(1), 53-76.

Cobb, G. W. (1978). The problem of the Nile: conditional solution to a change point problem. Biometrika, 62, 243-251.

Cox, D. R. ; Hinkley, D. (1974). Theoretical Statistics, Chapman and Hall, London.

Cox, D. R. ; Oakes, D. (1984). Analysis of Survival Data, Chapman and Hall.

Gelfand, A. E. ; Smith A. F. M. (1990). Sampling-based approaches to calculating marginal densities. Journal of the American Statistical Association, 85, 398-409.

Gelman, A. E. ; Rubin, D. (1992). Inference from iterative simulation using multiple sequences. Statistical Science, 7, 457-472.

Ghosh, J. K. ; Joshi, S. N. ; Mukhopadhyay, C. (1993), A Bayesian approach to the estimation of change-point in a hazard rate, Advances in Reliability (ed. por A. P. Basu), 141-170.

Green, P. J. (1995), Reversible jump Markov chain Monte Carlo computation and Bayesian model determination. Biometrika, 82(4), 711-732. 
Henderson, R. ; Matthews, J. N. S. (1993), An investigation of changepoints in the annual number of cases of hemolytic-uremic syndrome. Applied Statistics - Journal of the royal. statistical society, 42(3), 461-471.

Jarrett, R. G. (1979), A note on the intervals between coal-mining disasters_Biometrika, 66(1), 191-193.

Kaplan, E. L. ; Meier, P. (1958). Nonparametric estimation from incomplete observations. Journal of the American Statistical Association, 53, 457-481.

Kass, R. E. ; Tierney, L. ; Kadane, J. B. (1986). Laplace's Method in Bayesian Analisys. National Science Foundation.

Lee, A. F. S. ; Heghinian, S. M. (1977), A shift of the mean level in a sequence of independent normal random variables. Technometrics, 19, 503-506.

Lee, C. B. (1996), Nonparametric multiple change-point estimators. Statistics \& Probability Letters, 27(4), 295-304.

Lee, C. B. (1998), Bayesian analysis of a change-point in exponential families with applications. Computational Statistics \& Data analysis, 27, 195-208.

Maguire, B. A. ; Pearson, E. S. ; Wynn, A. H. A. (1952), The time intervals between industrial accidents. Biometrika, 39, 168-180.

Matthews, D. E. ; Farewell, V. T. (1982). On testing for a constant hazard against a change-point alternative. Biometrics, 38, 463-468.

Matthews, D. E. ; Farewell, V. T. ; Pyke, R. (1985), Asymptotic score statistic processes and tests for constant hazard against a change-point alternative. Annals of Statistics, 13, 2, 583-591.

Raftery, A. E. ; Akman, V. E. (1986), Bayesian analysis of a Poisson process with a change-point. Biometrika, 73(1), 85-89.

Smth, A. F. M. (1980), Change-point problems: approaches and applications. In Bayesian Statistics (eds J. M. Bernardo, M. H. DeGroot, D. V. Lindley and A. F. M. Smith) pp. 8389. Valencia University Press.

Smith, A. F. M. ; Roberts, G. O. (1993), Bayesian computations via Gibbs Sampler and related Markov Chain Monte Carlo methods (with discussion). Journal of Royal Statistical Society, B, 55, 3-23.

Stephens, D. A. (1994), Bayesian retrospective multiple-changepoint identification. Appl. Statist. , 43(1), 159-178.

Tierney, L. ; Kadane, J. B. (1986), Accurate approximations for posterior moments and marginal densities. Journal of the American Statistical Association, 81, 82-86.

West, R. W. ; Ogden, R. T. (1997), Continuous-time estimation of a change-point in a Poisson process. Journal of statistical computation and simulation, 56(4), 293-302. 